
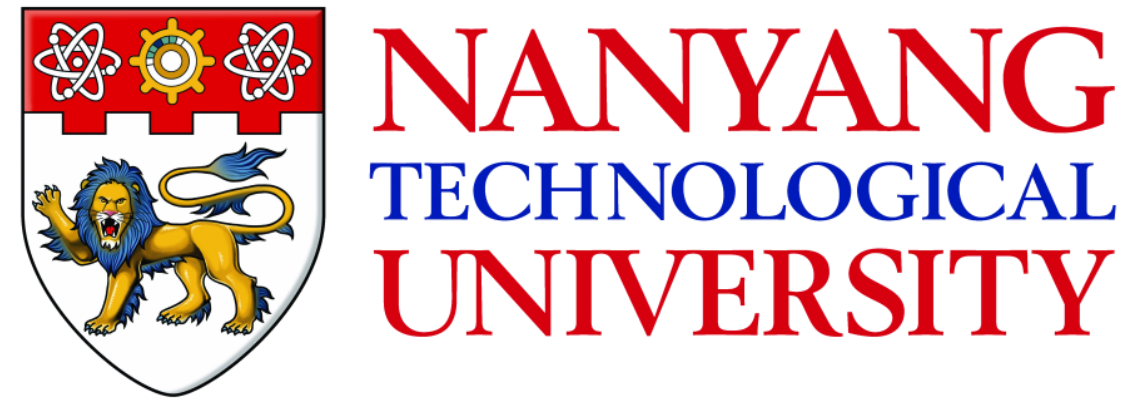

MICROFLUIDIC STUDIES ON FLOW MANIPULATION TO ASSIST METASTASIS RESEARCH

TRAN DUC QUANG SCHOOL OF MECHANICAL AND AEROSPACE ENGINEERING 2017 



\title{
MICROFLUIDIC STUDIES ON FLOW MANIPULATION TO ASSIST METASTASIS RESEARCH
}

\author{
TRAN DUC QUANG
}

School of Mechanical and Aerospace Engineering

A thesis submitted to the Nanyang Technological University in partial fulfilment of the requirement for the degree of

Doctor of Philosophy

\section{7}




\section{Abstract}

Cancer metastasis is initiated by cancer cells detaching from the primary tumor. This detachment process is driven by a complex combination of biophysical and biochemical effects. Among these, interstitial flow resulted from the intratumoral environment has been proven to be one of the causes assisting the directions of cell detachment and migration in tumor environment. This detachment leads to circulating tumor cells (CTCs) in vasculature or lymphatics, which can result in an uncontrollable spread of malignant tumors in the body. The appearance of CTCs is crucial for early detection of metastasis. In this study, we take advantages of microfluidics on flow manipulation to target the CTC isolation and characterize interstitial flow in a model tumor environment.

Isolation of CTCs has been challenging because of their low abundance and limited timeframes of expressions of relevant cell characteristics. In this work, we devise a novel hydrodynamic mechanism to sequentially trap and isolate floating cells in biosamples. We develop a microfluidic device for the sequential isolation of floating cancer cells through a series of microsieves to obtain up to $100 \%$ trapping yield and $>95 \%$ sequential isolation efficiency. Furthermore, we investigated the functional range of flow-rates for effective sequential cell isolation by taking the cell deformability into account. We verify the cell isolation ability using a human breast cancer cell line MDA-MB-231 with a perfect agreement with the microbead results. We further demonstrate that this device can be applied to isolate the largest particles from a sample containing multiple sizes of particles, revealing its possible applicability in isolation of circulating tumor cells in cancer patients' blood.

Additionally, we introduce a microfluidic platform to characterize multiple interstitial flow profiles through a tumor modelled by a cellular aggregate. Our findings 
demonstrate that the cellular aggregate behaves under the interstitial flow following two regimes: poroelasticity and solid plasticity, which are separated at a transient point, corresponding to fracture happened inside the cellular aggregate. We further characterize this transient point and find that the critical pressure at the transient point depended on the different loading rates of the flow. Experimental results show that the higher loading rate of the flow results in a higher critical pressure for aggregate fracture. We also develop a simplified theoretical model using poroelasticity to represent the cellular aggregate behavior within the elastic limit.

These studies have shown the potential of microfluidics in application on metastasis research with the flexibility of experimental setups. Our findings contribute to metastasis early detection through high efficiency CTC isolation and better understanding in the effects of interstitial flow on cancer tumor environment. 


\section{Acknowledgements}

My deepest gratitude is for my supervisors, Prof. Marcos (School of Mechanical \& Aerospace Engineering, Nanyang Technological University - NTU, Singapore) and Prof. David Gonzalez-Rodriguez (University of Lorraine, Metz, France). Prof. Marcos has been helping me since my first day in NTU. He is not only my advisor, but he also fights with me through all my difficulties in my $\mathrm{PhD}$ life. My project was inspired by Prof. Gonzalez-Rodriguez. Although he cannot literally fight with me in my difficulties due to our far distance, his advice, suggestion and comments are always valuable. Words might not be enough to describe my thankfulness to them.

I am especially grateful to Prof. Raymond H. W. Lam (City University of Hong Kong), Dr. Kong Tian Fook (NTU) and Hu Dinglong (City University of Hong Kong) for their great effort and support in the project of sequential isolation of floating cancer cells under continuous flow.

I would like to express my gratitude to Prof. Chian Kerm Sin, Sandy (School of Mechanical \& Aerospace Engineering, NTU) and Prof. Li Hoi Yeung (School of Biological Science, NTU) for their support as my Thesis Advisory Committee members.

It is very fortunate for me to be in Marcos Research Team, a very joyful and motivated group of research. Thanks so much to my colleagues, Dr. Kong Tian Fook, Dr. Yao Xin, Dr. Tran Ngoc Phu, Koh Boon Yong James, Xinhui Shen, Pei Yen Tan and Benjamin Tay for giving me encouragement and great support.

I would also like to thank Dr. Nuttawut Lewpiriyawong for helping me with microfabrication techniques and Dr. Chan Wing Yue for his helpful advice on biochemical issues. Thanks to my friends, Dr. Phan Dinh Tuan and Vo Xuan Quoc for their 
assistance and fruitful discussion during my work in Thermal and Fluid Lab (MAE, NTU).

During my research, I worked in Biological Lab (MAE, NTU) most of the time. My work cannot be completed without the help and instruction from Mdm. Heng Chee Hoon (the technician of the Lab) and Dr. Ling Siang Hooi. I really appreciate their assistance in laboratory facilities handling and safety assessment.

I am also indebted to Nanyang Technological University and School of Mechanical and Aerospace Engineering as involving me in NTU Research Program and providing me with the NTU Research Scholarship.

Last but not least, I give all gratefulness to my beloved family as always being my motive, heartening me up every time I need it.

Thanks to the four amazing years. 


\section{List of Figures}

Figure 1.1 Metastasis process. The development of malignant tumor encourages the massive growth of vasculature and results in the detachment of cancer cells out of the tumor. The tumor cells migrate through ECM and escape to blood/lymphatic circulation systems. They can travel in circulation systems and potentially settle secondary tumors at a distant organ. Reprinted by permission from Macmillan Publishers Ltd: Nature Reviews Cancer, Wirtz et al. [8], Copyright 2011

Figure 1.2 Tumor cells detached from primary tumor and circulating in blood circulation system before forming a secondary tumor at a distant organ. Figure is reproduced from [66], retrieved at http://vortexbiosciences.com/technology/.

Figure 1.3 Fluid pathways in the tumor microenvironment. Adapted by permission from Macmillan Publishers Ltd: Nature Reviews Cancer, Swartz et al. [95], Copyright 2012. 36

Figure 1.4 The schematic describes the autologous chemotaxis mechanism in tumor microenvironment when interstitial flow creates the CCL21 chemokine gradients guiding the tumor cells to lymphatic vessels. Adapted from Cancer Cell, Shields et al. [76], Copyright 2007, with permission from Elsevier.

Figure 2.1 Microfluidic designs for cell isolation. (a) Cell isolation method by Dhar et al. [108] using inertia effect in microvortices to trap cells through a series of expansion reservoirs. This method can achieve the isolation efficiency of $\sim 65 \%$. (b) Microfluidic device from Hou et al. [71] separating CTCs from blood with a spiral channel. They achieve a high separating efficiency of $85 \%$. (c) Conical-shaped microfilters from a microfluidic design of Tang et al. [109] can capture cells with high efficiency of $\sim 90-95 \%$. (d) Cell sorting technique by Mernier et al. [106] applying dielectrophoresis but it may trigger cell responses and alterations. (e) Cell isolation method by a vast of microtrappers developed by Chen et al. [110].

Figure 2.2 Theoretical models to characterize interstitial flow in cancer tumors. (a) The pressure profile and (b) interstitial flow velocity profile inside a tumor, as predicted by the mathematical model of Jain et al. [91]. This model shows that the pressure is highest at the center of the tumor and it drives the interstitial flow out to the tumor periphery. (c) The numerical model by Mohammadi et al. [121] showing the interstitial fluid pressure distribution inside a tumor. Arrows show the direction and magnitude of interstitial fluid flow. (d) 3D numerical model for interstitial fluid pressure contours of the spherical tumor surrounded by a spherical normal tissue conducted by Moghadam et al. [120]. 
Figure 2.3 Assays from Roger Kamm's group, where they used microfluidics to generate interstitial flow through type I collagen gel scaffold. They characterized interstitial flow through the gel scaffold by using two reservoirs with different levels of medium at two ends of the gel. (a) The gel scaffold design in Kalchman et al. [52]. (b) Microfluidic design by Polacheck et al. on direction of tumor cell migration through a matrix of collagen gel [44]. (c) Flow configurations for static cultures (c1), differential pressure gradient (c2), and generating physiological levels of shear stress (c3) [57].

Figure 2.4 The microfluidic device of (a) Melody Swartz's with design of the butterfly-shaped flow chamber [45] and (b) Mingming Wu's group to investigate the major phenotype of cell motility in collagen gel [61]. 50

Figure 2.5 Studies on cellular aggregates. (a) Guevorkian et al. $[134,135]$ measured surface tension of the cellular aggregate by micropipetting aspiration. (b) Gonzalez-Rodriguez et al. investigated aggregate fracture induced by a constant pulling velocity [138]. (c) Tissue spreading assays by Douezan et al. [136, 137] indicate that the wetting behaviors of cellular aggregates are governed either by the competition between cell-cell adhesion vs cell-substrate adhesion or by substrate rigidity. These phenomena demonstrate cellular aggregates behave as both liquids and solids. This figure is introduced from the review of Gonzalez-Rodriguez et al. [139] summarizing tissue mechanical behavior. 53

Figure 3.1 (a) Design of the sequential isolation microdevice for floating cells. (b) Configuration of the microsieve positions. With the lateral 'initial offset' from the center of the flow channel, the first floating cell be trapped in the first sieve; and the second cell would predominantly bypass through the negative $y$-side of the first sieve and be trapped at the second sieve position with a lateral 'sieve offset'. (c) The working principle of sequential cell trapping. Adapted from Tran et al. [56] with permission of The Royal Society of Chemistry.

Figure 3.2 Microfluidic design to characterize interstitial flow with constant flow rates. The device consists of five microchannels connecting to a central chamber. There are two designs for the chamber: chamber with pillars for hydrogel assays (top right) and without pillars for cellular aggregate assays (bottom right). The device is connected with two syringe pumps and two manometers during experiments. Dimensions are in $\mu \mathrm{m}$.

Figure 3.3 (a) Schematics of the second design with the side channel in parallel with the cell chamber. Dimensions are in $\mu \mathrm{m}$. (b) The equivalent electric circuit to the second design. Here, $R_{I}$ is the resistance of the cell chamber, $R_{2}$ is the resistance of the side channel. Being parallel to each other, the resistances receive the same voltage, and similarly the channels receive the same pressure difference. 
Figure 3.4 Cell isolation device fabrication procedure. (a-c) photolithography with AZ-9260 to deposit a positive photoresist pattern on a wafer. (c-d) Deep-Reactive-Ion-Etching to engrave the designed pattern onto the silicon master. (e-f) Produce the PDMS master by two-stage replica molding. (g-h) Casting PDMS, and plasma bonding with a glass slide to produce the final device.

Figure 3.5 Schematics of microfabrication procedure for cellular aggregate microfluidic device. (a-c) Photolithography process with negative photoresist SU-8 2100 to deposit a pattern of $\sim 100$ $110 \mu \mathrm{m}$ in thickness. (d-e) Soft lithography process with PDMS bonding with a glass slide to form a microfluidic device

Figure 3.6 Cellular aggregates formed by orbital shaking. (a) Spheroidal cellular aggregates with dimensions ranging from $50-450 \mu \mathrm{m}$ in diameter. (b) Isolated view of a single cellular aggregate that was selected for experiments.

Figure 4.1 (a) Velocity profile at the mid-height plane around a microsieve structure at the flow rate of $100 \mu \mathrm{min}^{-1}$. (b) dimensionless unidirectional factor $\varepsilon_{v}$ as a function of $x_{\text {sieve }}$. Scale bar: $200 \mu \mathrm{m}$. Error bars are standard deviations. Adapted from Tran et al. [56] with permission of the Royal Society of Chemistry.

Figure 4.2 (a) Sequential isolation process of microbeads. The first bead enters was trapped in microsieve 1; and subsequently the second bead bypassed the first filled trapper and was trapped in microsieve 2. This process continued until all the trappers are filled in a sequential manner. Trace of the bead movements was highlighted in red. Scale bar: $200 \mu \mathrm{m}$. (b) Trapping rate as a function of sieve offset for the 'enlarged device'. Error bars are standard deviations. $N \geq 5$. Adapted from Tran et al. [56] with permission of the Royal Society of Chemistry.

Figure 4.3 Sequential isolation of microbeads with a $20 \mu \mathrm{m}$ diameter in micro-sieves (top to bottom) using a 'true-scale' microdevice. Scale bar: $100 \mu \mathrm{m}$. Adapted from Tran et al. [56] with permission of the Royal Society of Chemistry.

Figure 4.4 Isolation of $80 \mu \mathrm{m}$ microbeads from (a) the mixture of $80 \mu \mathrm{m}$ beads and $20 \mu \mathrm{m}$ beads, and (b) the mixture of $80 \mu \mathrm{m}$ beads and $8 \mu \mathrm{m}$ beads, implemented on the enlarged device. Scale bar: $200 \mu \mathrm{m}$. Adapted from Tran et al. [56] with permission of the Royal Society of Chemistry. 85

Figure 4.5 (a) Microscopic snapshots of cells trapped in 'true-scale' microsieves for different durations of encapsulation ( $0 \mathrm{~s}, 30 \mathrm{~s}$ and $30 \mathrm{~min})$ under different sample flow rates $\left(3 \mu 1 \mathrm{~min}^{-1}, 6\right.$ $\mu 1 \mathrm{~min}^{-1}$ and $\left.9 \mu 1 \mathrm{~min}^{-1}\right)$ corresponding with different average shear rates $\left(7775 \mathrm{~s}^{-1}, 15555 \mathrm{~s}^{-1}\right.$ and $23300 \mathrm{~s}^{-1}$ ). Scale bar: $50 \mu \mathrm{m}$. (b) Trapping rate of cancer cells MDA-MB-231 as a function of the 
sieve offset, ranging $9-11 \mu \mathrm{m}$. Error bars are standard deviations. $N \geq 5$. Adapted from Tran et al. [56] with permission of the Royal Society of Chemistry

Figure 4.6 Representative bright-field (upper) and fluorescence (lower) microscopic images of MDA cells after treatment of the live/dead cell viability assay. Presence of fluorescence signals for all the trapped cells indicates their viability. Scale bar: $50 \mu \mathrm{m}$. Reproduced from Tran et al. [56] with permission of the Royal Society of Chemistry

Figure 5.1 Our theoretical model using poroelasticity to explain the behavior of the cellular aggregate under the flow. (a) The model in the point of view of a porous media flow. It includes a homogeneous porous cylindrical tube with radius $R$ and permeability $K$ where $K$ is a function of $r$, as described by Equation (3.6). (b) The model in the point of view of elasticity. It is an elastic cylindrical tube of radius $R$ with an inner empty hole of radius $r$ and an outer layer of elasticity $E$. This hole of radius $r$ is a simplified representation of the total pores of the model. A pressure difference $\left(P-P_{0}\right)$ is applied through it. Assume that the deformation in all direction is equal. 92

Figure 5.2 Results from theoretical model of interstitial flow through a cylindrical poroelastic tube including a porous core with radius $r$ and an elastic cylindrical annulus region. The graphs of $\tilde{Q}$ and $\tilde{r}$ versus $\Delta \tilde{P}$ are plotted with (a) $\tilde{p}_{0}=0$ and (b) $\tilde{p}_{0}=0.5$

Figure 5.3 The schematics describes the working principle of the first design of microfluidic system to generate constant flow rates. K1 and K2 are the tubing clamps. K1 and K2 are unlocked for "no flow" condition. When using Pump 2 to move a cellular aggregate into the chamber, K1 is locked and $\mathrm{K} 2$ is unlocked. When applying flow through the main channel, both $\mathrm{K} 1$ and $\mathrm{K} 2$ are locked. Note: A 'locked' clamp means that the tubing is disconnected. 100

Figure 5.4 Experimental results of device calibration for the first design with type I collagen gel. (a) The images of collagen gel rigidified inside the chamber, (left) the gel in square chamber is made from $3 \mathrm{mg} \mathrm{ml}^{-1}$ gel solution; (top right) the $2 \mathrm{mg} \mathrm{ml}^{-1}$ gel solution was shaped in rectangular chamber; (bottom right) the $2 \mathrm{mg} \mathrm{ml}^{-1}$ gel solution was shaped in square chamber; scale bar is $500 \mu \mathrm{m}$. (b) The increase of pressure difference to a constant value at steady state when a constant flow rate of $150 \mathrm{nl} \mathrm{min}^{-1}$ was applied through a $3 \mathrm{mg} \mathrm{ml}^{-1}$ square gel chamber (c) The linear relation between pressure difference and flow rate across the $3 \mathrm{mg} \mathrm{ml}^{-1}$ gel chamber at each steady state, showing the hydrodynamic resistance of the collagen gel. From here, using Darcy's law equation, we calculate the permeability of this collagen gel is $1.62 \times 10^{-13} \mathrm{~m}^{2}$. (d) The comparison of hydrodynamic resistance between collagen gel in square shape $(500 \mu \mathrm{m} \times 500 \mu \mathrm{m})$ and rectangle shape $(500 \mu \mathrm{m} \times 1000 \mu \mathrm{m})$. Two different shapes of gel resulted in 2 different resistance values, but the permeability is the same due to the same batch of $2 \mathrm{mg} \mathrm{ml}^{-1}$ gel $\left(6.11 \times 10^{-13} \mathrm{~m}^{2}\right.$ and 
$5.17 \times 10^{-13} \mathrm{~m}^{2}$, respectively). Note: the fitting curves in the graph have a linear function of $y=a+b x$

Figure 5.5 The fibronectin coating quality test. Fluorescent intensity was measured at 5 regions using ImageJ (Region 1 is the non-fluorescent background). The microchannels coated with a fluorescent fibronectin solution (bovine fibronectin conjugated with HiLyte488) (left) showed the higher fluorescent intensity than the microchannels only coated with FITC fluorescein dye solution (right). This result revealed the appearance of fibronectin made the fluorescein dye remain in the microchannel surfaces. Scale bar: $200 \mu \mathrm{m}$

Figure 5.6 A cellular aggregate moved into the central chamber. (a) The cellular aggregate was partially dragged into channel 3. (b) The cellular aggregate was slowly pushed to move inside channel 3. (c) The aggregate reached the chamber. (d) After the heating stage was set at $37^{\circ} \mathrm{C}$, the aggregate adhered to channel walls. The red circles are the signs of the cells spreading onto the channel walls 106

Figure 5.7 Experimental results with cell aggregates (a) An image captured from the movie of flow through a MCF-7 cellular aggregate inside the cell chamber, right after fracture occurred. The flow with fluorescein was observed to advance progressively and very slowly from the left side towards the right side and then, suddenly, to reach all the way to the output (b) The increase of pressure across the cell aggregate when we applied flow at $4 \mu \mathrm{m} \mathrm{s}^{-1}$. The pressure achieved a constant value when the system reached the steady state. (c) The pressure difference across the cellular aggregate chamber increased to a highest value then dropped when the flow velocity kept increasing. The error bars are standard deviations. We hypothesize that the cellular aggregate behaves as two regimes: poroelasticity and solid plasticity. These two regimes are separated by a "breaking point" when the fracture happened inside the cellular aggregate

Figure 5.8 The experimental results showing the problem with unstable pressure readings at low flow velocity. (a) The pressure difference was observed to drop after 1600 seconds at flow velocity of $0.2 \mu \mathrm{m} \mathrm{s}^{-1}$. (b) A test run with water inside a closed microchannel system, at $25^{\circ} \mathrm{C}$. The pressure was constant for 1600 seconds. (c) A test run with water inside a closed microchannel system, at $37^{\circ} \mathrm{C}$. The pressure was observed to drop linearly. (d) A test run with water inside a closed microchannel system, at $37^{\circ} \mathrm{C}$. The device was then submerged in distilled water and the pressure was observed to increase to a constant value.

Figure 5.9 The graph shows the calibration results when applying linear increasing flow rate at (a) loading rate 1 with increase rate of $1 \mu 1 \mathrm{~min}^{-2}$ and (b) loading rate 2 with increase rate of $0.5 \mu \mathrm{min}^{-2}$. The hydrodynamic resistance of the side channel to culture medium was measured and calculated at $32.18 \mathrm{~Pa}_{\min } \mu \mathrm{l}^{-1}$. 
Figure 5.10 Experimental results with cellular aggregates in the second design. (a) The image of the cellular aggregate sitting inside the chamber at $37^{\circ} \mathrm{C}$. (b) The fracture happened inside the cellular aggregate when the flow was applied. (c) The graph shows how the pressure difference changed over time while increasing the flow rate. (d) The equivalent resistance of the cellular aggregate parallel with the side channel was calculated. The figure shows a sudden drop in resistance when fracture occurs.

Figure 5.11 The graph shows the breaking points of cellular aggregates at two different loading rates. Loading rate 1 at $1 \mu 1 \mathrm{~min}^{-2}$, loading rate 2 at $0.5 \mu 1 \mathrm{~min}^{-2}$. Each loading rate was run with 5 cellular aggregates of the same batch. The error bars are the standard deviations.

Figure 5.12 The deformation of cellular aggregates under two loading rates. Loading rate 1 at $1 \mu 1 \mathrm{~min}^{-2}$, loading rate 2 at $0.5 \mu 1 \mathrm{~min}^{-2}$. The cellular aggregate under loading rate 1 was more damaged than under loading rate 2 . The time scale for the flow to damage the fracture appearance under loading rate 1 was also shorter than loading rate 2 .

Figure 6.1 Proposed design to characterize flow through a cellular aggregate (red chamber) placed between two chambers of collagen gel (blue chamber)

Figure 6.2 Proposal of an integrated device including the microfluidic design to generate interstitial flow through a cellular aggregate associated with extracellular matrix, connected to an isolation channel to capture the single cells escaped from the tumor. 134

Figure A.1 Schematics of the preparation for the calibration test of the modified design to characterize interstitial flow through a cellular aggregate 


\section{List of Tables}

Table 3-1 Summary of surface treatment procedures used in the whole project ........................... 72

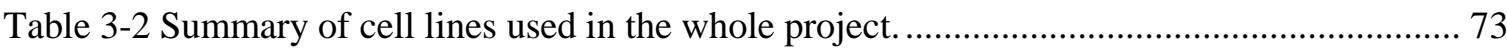




\section{List of Abbreviations}

CTCs

DNA

ECM

IFP

MEMS

PDMS

FRAP

MRI

FITC

PBS

DMEM

FBS

MCF-7

DMSO
Circulating Tumor Cells

Deoxyribonucleic Acid

Extracellular Matrix

Interstitial Fluid Pressure

Microelectromechanical systems

Polydimethylsiloxane

Fluorescent Recovery After Photobleaching

Magnetic Resonance Imaging

Fluorescein-5-isothiocyanate

Phosphate Buffered Saline

Dulbecco’s Modified Eagle Medium

Fetal Bovine Serum

Michigan Cancer Foundation-7

Dimethyl sulfoxide 


\section{Table of Contents}

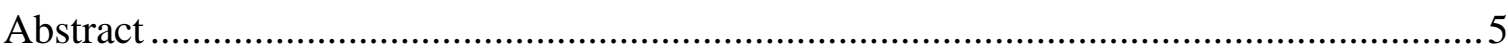

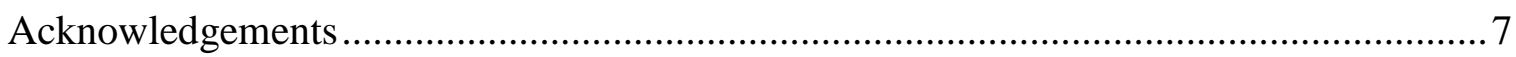

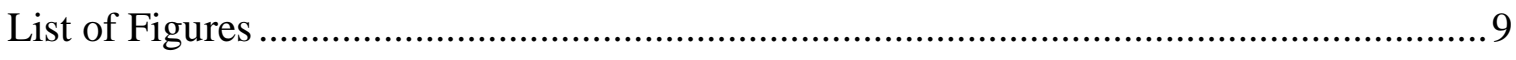

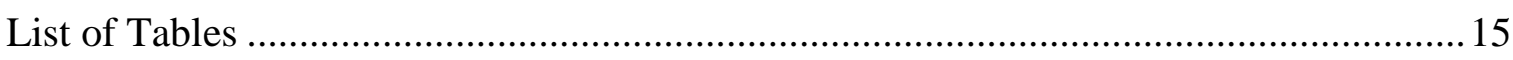

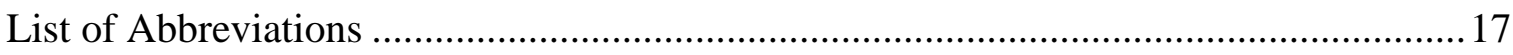

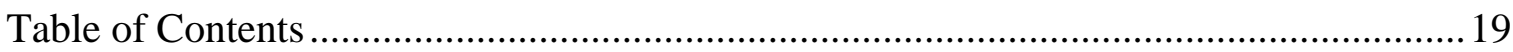

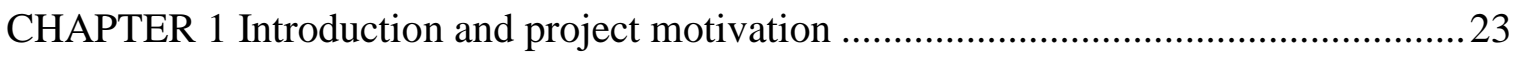

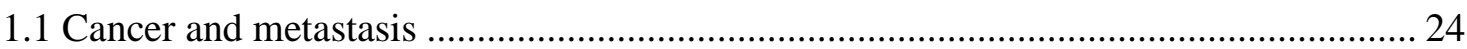

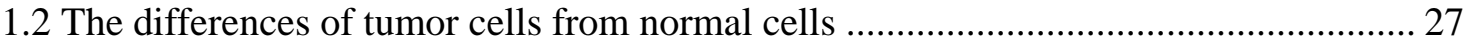

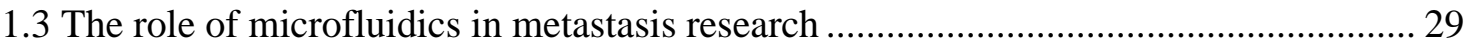

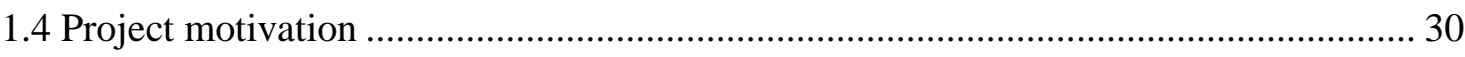

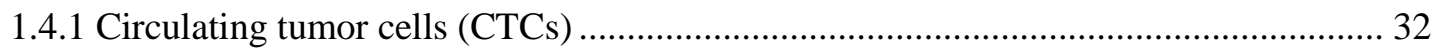

1.4.2 Interstitial flow and its effects in the tumor environment .......................................... 34

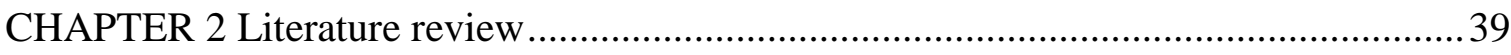

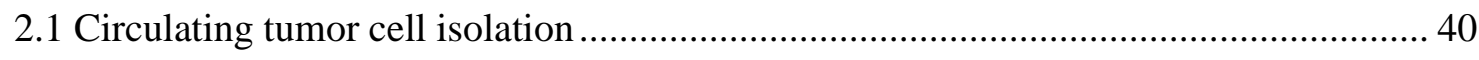

2.1.1 Previous studies using microfluidics on CTC isolation............................................. 40

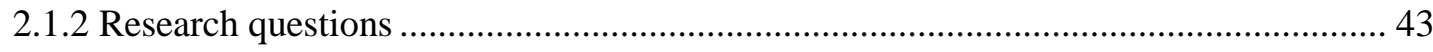

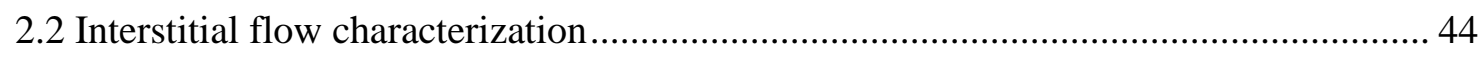

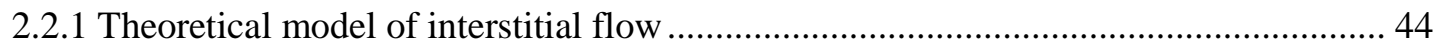

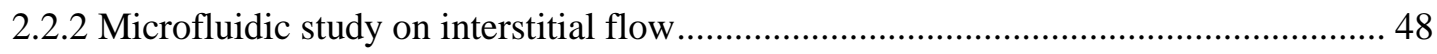

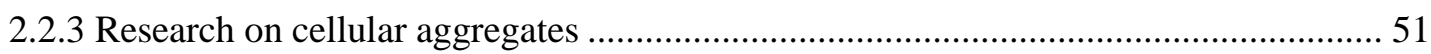




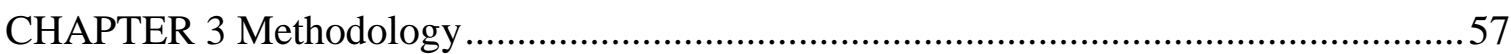

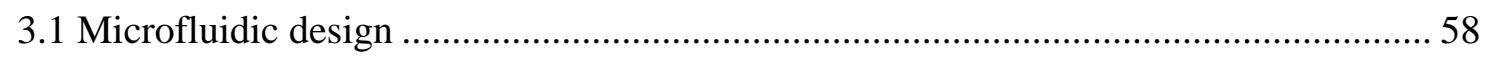

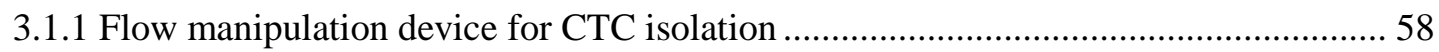

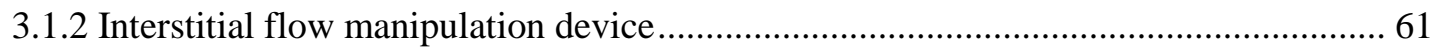

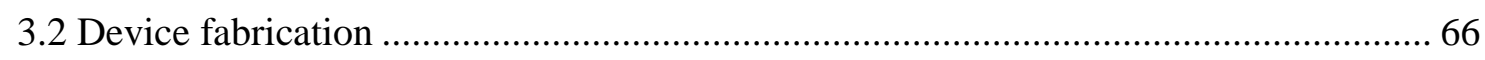

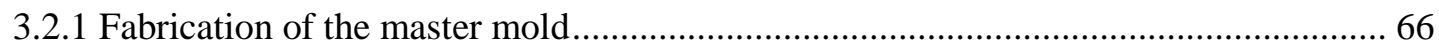

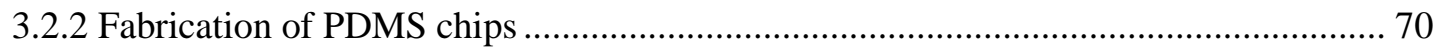

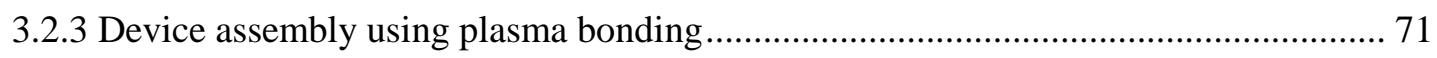

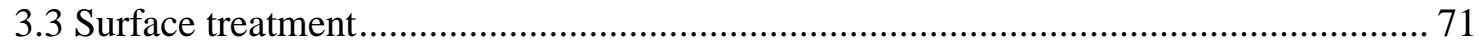

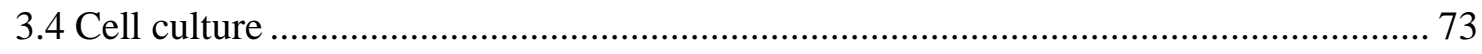

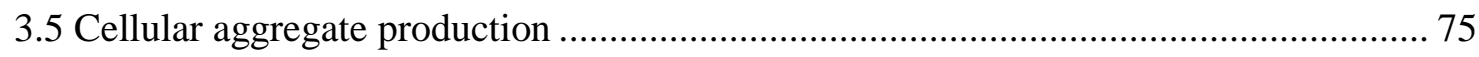

CHAPTER 4 Sequential isolation of floating cancer cells under continuous flow ........... 77

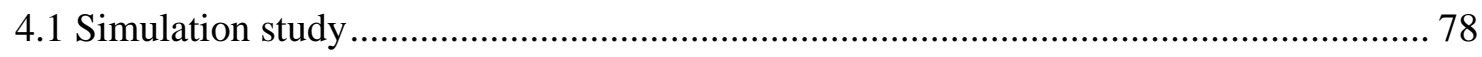

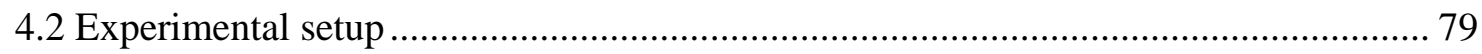

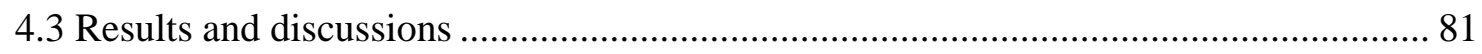

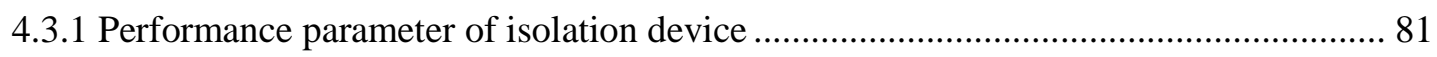

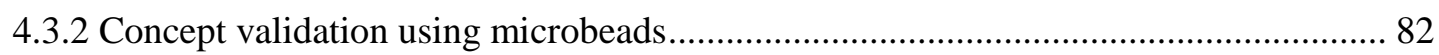

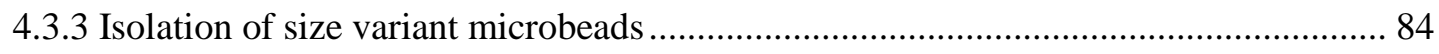

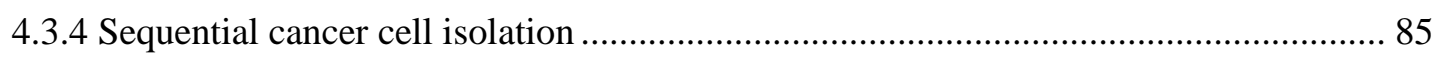

CHAPTER 5 Microfluidic study of interstitial flow through a model tumor....................91

5.1 Theoretical poroelastic model for flow through a cellular aggregate ........................... 92

5.2 First microfluidic design to characterize interstitial flow with constant flow rates ...... 98 
5.2.1 Experimental setup and working principle.

5.2.2 Calibration of the device with flow through an inert medium ................................ 101

5.2.3 First experiment with cellular aggregates............................................................. 104

5.2.4 Limitation of the microfluidic device for interstitial flow with constant flow rate .... 111

5.3 Second microfluidic design to characterize interstitial flow with continuously increasing flow rate

5.3.1 The advantages of the second design

5.3.2 Calibration of the new device

5.3.3 Second experiment with cell aggregates

5.4 Discussion

5.4.1 Simple microfluidic setup for interstitial flow characterization

5.4.2 Behaviors of cellular aggregates under interstitial flow 120

5.4.3 The dependence of cellular aggregate behavior on the flow loading rate

5.4.4 Validation of theoretical poroelastic model for the first regime of cellular aggregate behavior under interstitial flow . 122

CHAPTER 6 Conclusion and perspectives 125

6.1 Conclusion. 126

6.1.1 The flexibility of microfluidics on flow application in metastasis research. 126

6.1.2 The sequential isolation of floating tumor cells under continuous flow 126

6.1.3 The characterization of interstitial flow through a model tumor. 127

6.2 New perspectives. 128

6.2.1 Enhancement of CTC isolation ability in inertia-based sorting devices by combination of the sequential isolation device 128 
6.2.3 Improvement of the microfluidic design to characterize interstitial flow through the cellular aggregate with the ability of quantifying the aggregate permeability ..... 130

6.2.4 Characterizing flow through a cellular aggregate with an extracellular matrix 131

6.2.5 Developing theoretical model for two regimes of cellular aggregate behaviors 132

6.2.6 Investigation on the interdependence of interstitial flow effects and biochemical environment changes to a tumor 132

6.2.7 Integrated microfluidic device to quantify cancer cell detachment from a primary tumor 133

6.2.8 Overall vision and further approaches of the project 134

References

Appendix

Publication 153 


\section{CHAPTER 1 \\ Introduction and project motivation}

Every research project is inspired by some specific reasons. This PhD thesis begins by introducing about what motivated us to work on microfluidics and cancer research. This chapter gives the readers some basics about cancer and metastasis. We are going to find out why cancer is one of the top leading causes of death in the world.

The benefits of microfluidics on cancer research are also discussed. We also present our approach to metastasis research and why we choose microfluidics as a tool in our experiments.

At the last part of this chapter, the detailed questions in this project are focused. We are going to present two research topics that were targeted during this study: 'Sequential isolation of floating cancer cells under continuous flow' and 'Microfluidic study for interstitial flow through a model tumor'. 


\subsection{Cancer and metastasis}

We generally refer to cancer as one of the most dangerous group of diseases, which causes millions of deaths in the world [1]. Cancer is harmful not only because it is caused by many unidentified factors, but also because people having cancer at early stage sometimes do not feel any symptoms, or they usually underestimate those symptoms.

So, what is cancer and why is it fatal?

Human body is made of billions of cells. Those cells are arranged and connected together in order to form many groups of cells called tissues or organs. Different parts of body contain different types of cells, which have their own shapes. But basically all human cells have a similar structure consisting of a nucleus at the center, surrounded by cytoplasm, and a membrane bounding around [2]. The nucleus contains long strands of DNA which possess a huge set of genes. The genes are the brain of cells. They constitute the blueprints for creating cell structures, and control all cellular activities [2]. For instance, they can decide when the cell reproduces itself, when the cell will die, and other cellular activities.

Even if the probability is low, when the body is affected by certain external or internal factors, some gene mutations may occur during cell division [3, 4]. The accumulation of such mutations can make cells operate in an uncontrollable way. For example, a gene regulating the continuous cell doubling may be permanently switched on while other genes limiting the life-span of the cells can be permanently off [5]. However, the fact is that the process of a normal cell becoming a cancer cell is not as easy as it is thought. There have to be several mutations before a cancerous phenotype is attained [6, 7]. When experiencing a mutation, cells are usually killed by the immune system or even 
by themselves; only a small number of the mutated cells can survive and eventually turn into cancer cells [6].

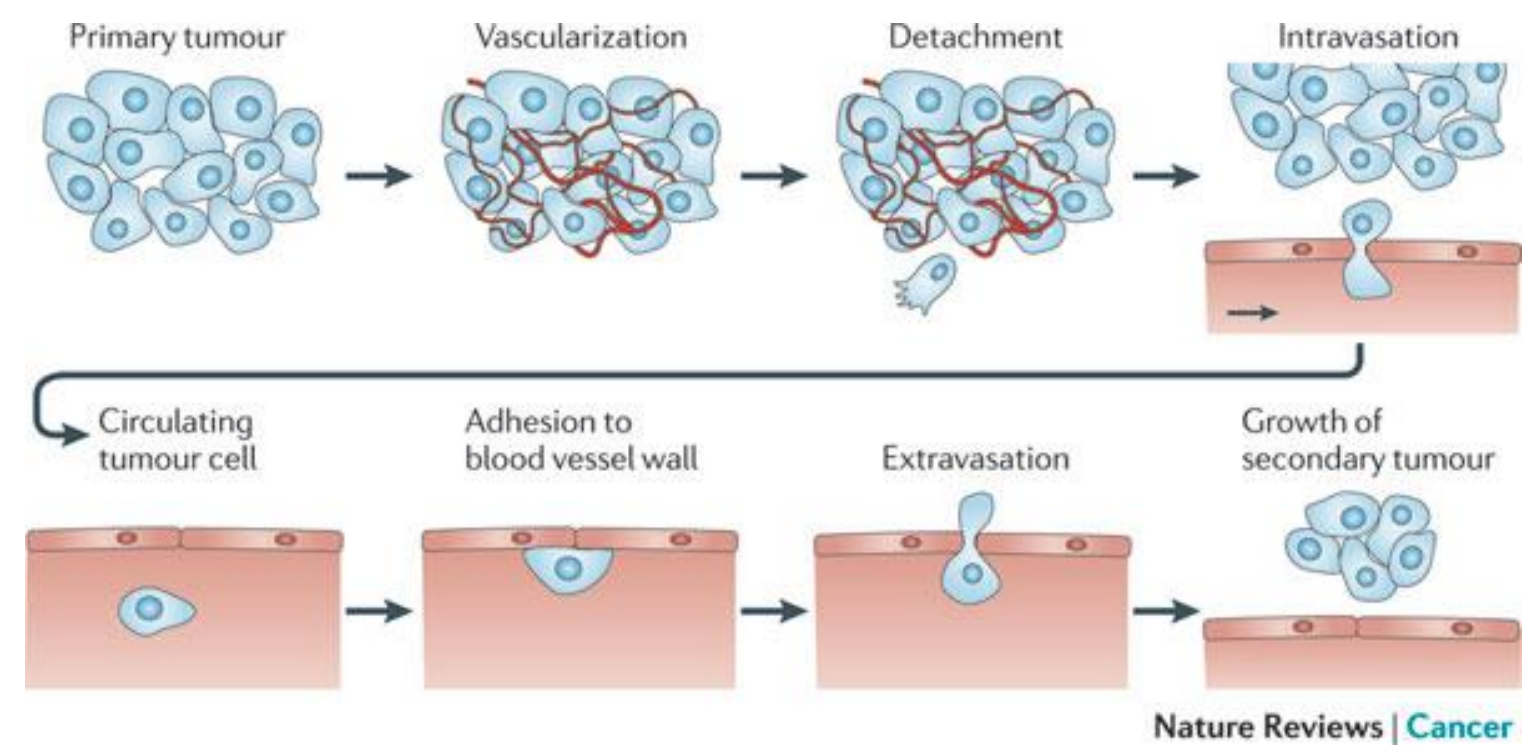

Figure 1.1 Metastasis process. The development of malignant tumor encourages the massive growth of vasculature and results in the detachment of cancer cells out of the tumor. The tumor cells migrate through ECM and escape to blood/lymphatic circulation systems. They can travel in circulation systems and potentially settle secondary tumors at a distant organ. Reprinted by permission from Macmillan Publishers Ltd: Nature Reviews Cancer, Wirtz et al. [8], Copyright 2011.

Those mutations induce characteristics of cancer cells that differ from those of normal cells. Cancer cells do not completely finish their growth but stay immature. Over many times of reproducing, they keep losing their genetic information [5]. This makes cancer cells reproduce more quickly and even badly-mutated. The incomplete reproduction can also make cancer cells lose the adhesion molecules on their surface that keep them attached to tissues nearby [9]. Therefore, cancer cells are less adhesive and able to potentially detach from their neighbors. In particular, the most important feature of cancer cells is that if being supplied enough nutrients, they can stop themselves from selfdestructing process, or they may self-destruct more slowly than reproducing $[10,11]$. So the number of cancer cells keeps on increasing until they form a tumor. In some cases, they grow not too fast and form a big but still controllable lump called a benign tumor [12]. In other cases, when the genes are more damaged, a malignant tumor [12] is formed 
and developed to an oversized tumor. The tumor cells have potential to spread to surrounding tissues and even migrate to other parts of body. That migration process is called metastasis $[8,13,14]$ (Figure 1.1), which causes 90\% deaths of cancer patients [15].

Metastasis originates from cancerous cells in malignant tumors [13]. As the cells there do not stop reproducing, they make the tumor become oversized, and take up increasingly more space in the body. The tumor soon grows onto surrounding tissues. This is called an invasion process $[16,17]$. When the tumor grows, it will generate forces on the normal tissue nearby and block the blood vessels in this area. These cancer cells can promote the vascular system to form new capillaries to provide more nutrients for them, a process called angiogenesis $[18,19]$. Moreover, cancer cells are able to detach from their tumor under effects of many cellular factors and gradients. A separated tumor cell can go through vascular cells to the blood circulation, or it can cross the stroma and drain into lymphatic system (intravasation process [20, 21]). From this stage, that tumor cell travels randomly, and it is very difficult to predict the way and how far the cell can travel from the primary tumor. However, in the blood circulation, it is not easy for that circulating tumor cell to survive while being battered by fast blood flow or being killed by the white blood cells [22]. There are just a small number of cancer cells that can stick to the platelets, and form clumps to get more protection [23-27]. This combination helps the cancer cells travel further to other capillary network. The cells can be stuck at small blood vessels and escape through the vessel wall. If the cancer cell is traveling in the lymphatic system, it can attach at the small channel inside lymph nodes (extravasation process [28, 29]). After getting out of the blood circulation or being attached at a lymph node, that cell settles and continues growing to form a secondary tumor at the new site. 
Each metastatic cell has the potential to begin a new tumor when it settles in a new site. When the number of tumors are increasing, the angiogenesis becomes enormous, which depletes nutrients of the patient body [30]. Moreover, the overwhelming growth of the malignant tumors also damage and disrupt the functions of the host organs [31]. These can lead to death if the patients do not seek for treatment opportunely. Nevertheless, metastasis treatment is challenging because the cancer cells are the own cells of the patients. This implies that treatments directed against cancer cells will also harm normal cells in the patient's body. For example, chemotherapies are used to kill rapidly growing cancer cells. But they also kill the normal cells that grow rapidly such as hair and stomach lining. Then, the treatment must be enough to kill the cancer cells, but not that much to kill the patient. Early detection to diagnose precancerous changes or early stage cancer greatly increases the chance of successful and effective treatment $[32$, 33].

\subsection{The differences of tumor cells from normal cells}

The mutation happened in genes results in the abnormal behaviors of tumor cells. The alterations of proteins that control the cellular activities will cause the tumor cells to induce physical and chemical characteristics that are different from those of the normal cells.

\section{Physical difference}

The body tissues grow by cell division [2]. One cell doubles by dividing itself into two new cells. When the body is grown up, the cells will reproduce and die in a certain time to maintain the stable number of cells. That comes from the operation of the genes. They can sense the bio-chemical signals from surrounding environment or from the neighbor cells. The body tissues and organs can retain their shapes because the cells have 
ability to stick to their neighbor cells and extracellular matrices [2]. In a normal way, the cells have to stay at their provided place. Otherwise, they will die immediately when settling at a wrong place. In cancer cells, the damaged genes can make the cells multiply without stopping. The number of cancer cells keeps on increasing rapidly until they form an oversized tumor [12]. The tumor can invade and occupy increasingly more space in the body. The tumor soon grows onto surrounding tissues $[16,17]$. When the tumor grows, it will generate forces on the normal tissue nearby and block the blood vessels in this area.

\section{Cell adhesion}

Cell adhesion is the mechanism of a cell binding to a non-cellular surface like an extracellular matrix or linking to the neighbor cells, by using adhesion protein molecules such as selectins, integrins and cadherins [2]. Moreover, cell adhesion is very important for the cells to maintain the tissues' and organs' morphology or the multicellular structure [34]. Cellular adhesion also involves in bio-chemical signal transduction as the adhesion proteins are distributed on the surface of cells [35].

The mutations occurred in the reproduction of cells may make them become immature or incompletely grown. This results in faster reproducing time and lack of many cell molecules. After long time incomplete reproducing, the cells lose many proteins on their surface like cadherins, integrins [5]. Those cancerous cells must be less adhesive and can have potential to detach from the tumor [9]. The loss of cadherins happened in some processes, such as epithelial-mesenchymal transition (EMT) which is involved in the cadherin expression reduction contributing to embryonic development and tumor growth, $[20,36,37]$, will worsen the level of malignancy of the cells. These cancer cells can promote the vascular system to form new capillaries to provide more nutrients for them, a process called angiogenesis $[18,19]$. 


\subsection{The role of microfluidics in metastasis research}

The danger of metastasis was not previously well-estimated. A previous study in European Journal of Cancer in 2010 pointed out that only five percent of cancer research funds were spent on metastases, while in fact it killed 90 percent of all cancer patients [15]. Nonetheless, research on metastasis has become more imperative in recent years with tremendous findings.

The development of MEMS and microfluidic devices provides scientists with more opportunities and powerful tools to study small scale biological aspects. Microfluidics with many different designs can provide various types of microenvironment which can be used to mimic the human tissue and tumor environment in metastasis [3842]. Many experiments simulating the metastasis stages such as the migration [43-45], intravasation [46-48], and extravasation [49-51] of cancer cells, and experiments to study the effect of anti-cancer drugs $[52,53]$ have been carried out recently.

Microfluidic platforms have the ability of precisely controlling flows or biochemical and biophysical gradients. Microchannels in microfluidics contain fluid in microliter volumes and smaller volumes, which makes the fluid flow always be laminar with low Reynolds number [54]. In such regime, the gradients of molecules can be wellcontrolled. For example, the application of microfluidic syringe pumps provides the high precision of flow manipulation $[55,56]$. Pressure gradients across a flow chamber can be induced by a simple setup of medium reservoirs in different height levels $[57,58]$ or can be accurately controlled by a peristaltic pump [45].

Additionally, microfluidic devices are usually associated with microscope systems that can perform live imaging or monitor the flows and gradients in metastasis assays. For instance, many studies have been carried out on observation of the migration of tumor 
cells through an extracellular matrix scaffold $[44,45,52]$, the motility of tumor cells to enter the blood circulation [46] or screening the effects of anti-cancer drugs on tumor cells $[52,59,60]$. Using technologies like Fluorescence Recovery After Photobleaching (FRAP) and Magnetic Resonance Imaging (MRI) can help quantifying the velocity of interstitial flow through a collagen gel scaffold [44, 61].

Microfluidic devices usually require the use of a clean room for micro-fabrication and the application of polydimethylsiloxane (PDMS) molding. The materials involved in microfluidic devices are commonly PDMS polymers and glass, which are biocompatible and low cost [54]. With the development of extracellular matrix protein coating, microfluidics is even able to easily mimic the tumor microenvironment. For example, the coating of collagen gel solution on a PDMS chamber helps creating a 3D gel scaffold for seeding cells directly on chip, which results in a 3D structure of tissue or tumor [43, 52, 57]; the coating of Fibronectin proteins on a flat, rigid surface induces a strong adhesion of cells onto that surface, which supports the cell culture on flat surfaces $[62,63]$. However, this method also comes with challenges in dealing with small sample volumes, low cell number and small-scale impacts.

\subsection{Project motivation}

As mentioned earlier, the detachment of single cells from the primary tumor is the origin of metastasis. This metastasis process is affected by many biochemical gradients and physical factors. One of the most important factors for metastasis progression is fluid flow. In this project, we focus on the fluid mechanic perspective of two important factors that are involved in metastasis derivation, which are circulating tumor cells (CTCs) in blood circulation system or lymphatic system, and interstitial flow in tumor environment. CTCs moving inside blood capillary experience the blood flow in their external while 
interstitial flow is the fluid flow within the internal of the tissue or tumor. In general, we carry out experiments to manipulate and investigate the effects of both external and internal flow through cells or tumors.

Circulating tumor cells (CTCs) have the potential to settle secondary metastatic sites, which can result in an uncontrollable spread of malignant tumors in cancer patients. As CTCs were found to exist at early stages of the disease [64, 65], detection of CTCs is a robust method that can contribute to early stage cancer diagnosis and treatment. Since the external flow through a CTC is considered as Stokes flow moving through a sphere, we can manipulate the flow to control the cell movement so that the cells get trapped intentionally, thus helping the CTC detection.

Additionally, interstitial flow resulted from the intratumoral environment has been proven to be one of the causes assisting the directions of cell detachment and migration in tumor environment. This detachment and migration can lead to CTCs and metastasis spreading. Thus, understanding the effects of interstitial flow in the tumor environment is crucial for metastasis research.

The benefits of microfluidics in metastasis assays have provided us with solutions for studies on CTCs detection and interstitial flow characterization. Microfluidics is a particularly appropriate technique to precisely control flows and gradients, which is very helpful in studies on CTCs and interstitial flow.

In this study, we present our microfluidic development to target two topics:

1) Isolation of circulating tumor cells with high efficiency.

This study aims to introduce a novel strategy to sequentially trap and isolate rare floating cells, like CTCs, in biosamples with up to $100 \%$ efficiency. The 
outcomes of this study contributes to CTC detection in early cancer diagnosis, or they can be applied in particle isolating and sorting assays.

2) Characterizing interstitial flow through a cancer tumor.

Through this study, we aim to investigate the effects of multiple interstitial flow profiles on a cancer tumor, and the behaviors of the tumor under the flow. This study provides further insights of the influences of interstitial flow in the tumor environment relating to cell detachment and migration phenomena.

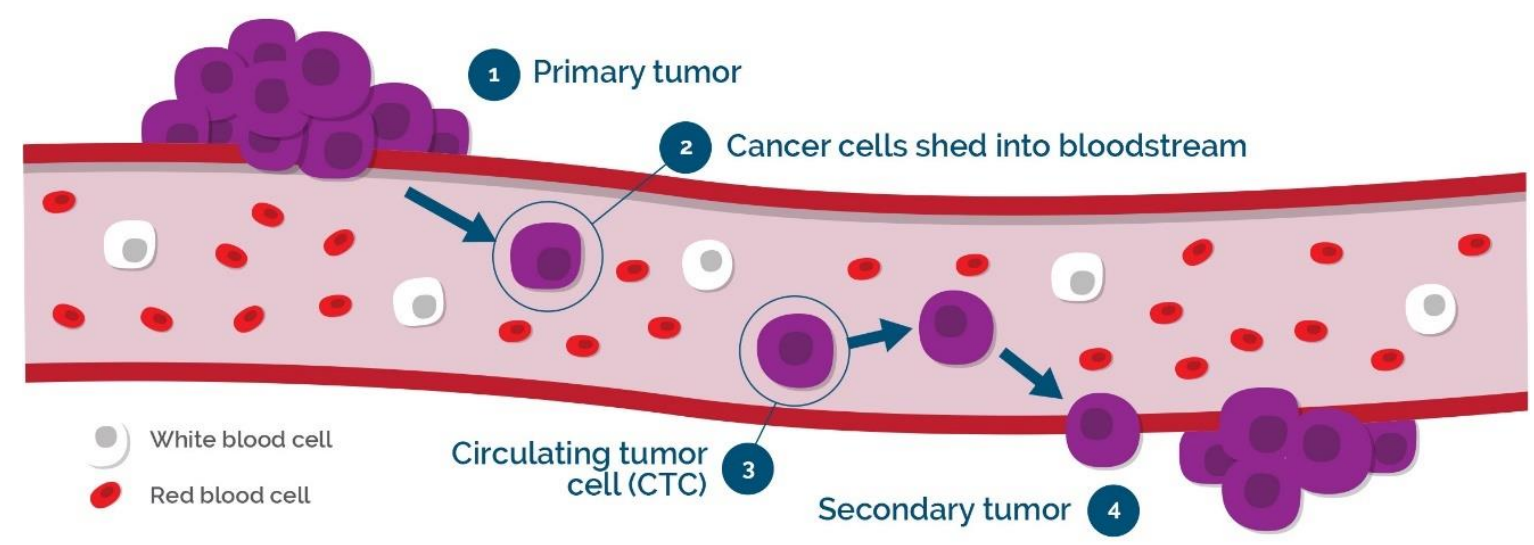

Figure 1.2 Tumor cells detached from primary tumor and circulating in blood circulation system before forming a secondary tumor at a distant organ. Figure is reproduced from [66], retrieved at http://vortexbiosciences.com/technology/.

\subsubsection{Circulating tumor cells (CTCs)}

Circulating tumor cells are single cells escaped from a primary tumor and traveling in the blood and lymphatic circulation systems (Figure 1.2). CTCs account for the subsequent growth of secondary tumors at new sites in patient body, which can become uncontrollable and lead to death [67].

Studies demonstrated that CTCs were found to exist at early stage of the disease $[64,65]$. Thus, CTC detection becomes a robust method for metastasis diagnosis to 
achieve more successful and effective treatment. Conventionally, the diagnosis of cancer requires surgical biopsy - a process of sampling cells or tissues from human body for examination. A part of the abnormal tissue is removed by either needles or surgery. The abnormal cells will then be analyzed and the CTC detection is indirectly indicated from analysis of the abnormal cells [68]. However, surgical biopsy cannot be performed frequently due to the invasive characteristic and the procedure may not be able to reflect current tumor dynamics and drug sensitivity [69]. On the contrary, the "liquid biopsy" involves finding the rare cells, such as the circulating tumor cells (CTCs), stems cells, and leukemic cells, in the peripheral blood for the diagnosis and prognosis of cancers $[69,70]$. However, the presence of CTCs is rare because they are either killed by the immune system or the high shear rate of the blood flow. In order to survive within the circulation systems, CTCs need to form a clump with platelets to get protected [23, 24]. These rare cells are naturally in low-abundance and typically in the order of $\sim 1$ to 100 rare cells per $10^{9}$ blood cells [71]. In other words, albeit pivotal for early metastatic cancer detection, recovering these rare cells from a patient's peripheral blood is like finding a needle in a haystack.

Nonetheless, the isolation and detection of CTCs are made possible with the advancement in microfluidics technology. The recent trends in microfluidics involve the integration of multiple traditional laboratory procedures into an automated micro total analysis system ( $\mu$ TAS) or lab-on-a-chip for a myriad of biological applications such as cancer screening, disease diagnosis, and drug test [72]. The miniaturization of these labon-a-chip devices offers the benefits of requiring less sample volume, faster analysis, precise fluid handling, and reduced biological and chemical wastes [73]. The main challenges in rare CTC isolation are in obtaining a high capture efficiency and high-level 
of statistical confidence for the detection of rare cells due to its low abundance nature [70].

\subsubsection{Interstitial flow and its effects in the tumor environment}

Metastasis is affected by many physical and bio-chemical factors. In addition to gene mutations inside individual cells or the lack of cell-cell adhesion within a malignant tumor, the tumor microenvironment has a big impact on cancer development as well. The environment biophysical factors such as forces, flows and gradients are increasingly regarded as to having significant effects on tumoral evolution. Among these biophysical phenomena, interstitial flow emerges to be the key factor that can alter the tumor microenvironment and play a central role in early stages of metastasis. Interstitial flow originated from interstitial fluid which exists within tissues, moving around cells and through the ECM's pores. Interstitial flow can affect the extracellular gradients [74-76]; apply mechanical effects to the cells or the ECM [77, 78], and transport antigens and soluble factors within the tumor environment [79].

In normal tissues, the hydrostatic and osmotic pressure differences between blood capillaries and local interstitial space, as well as the relative permeability of the interfaces between these different systems, result in a small fraction of fluid and macromolecules escaping from the capillaries and draining into tissue space. However, an estimated $90 \%$ of the exuded fluid is brought back to the post-capillary venules [80, 81]. As the effect of osmotic pressure differences, there is still a small amount of fluid remained and then absorbed by the lymphatic capillaries. Since blood and lymphatic capillary walls also have certain permeability to plasma proteins, the interstitial fluid turns to be lymph with nearly the same composition [80, 81]. The lymphatic capillaries with the initial and terminal sections working like one-way valves avoid the fluid from flowing reversely. 
They are connected to the ECM, receiving stresses to create the pressure needed, and then force lymphatic and interstitial flow through the tissue.

In cancer, tumor development brings about many alterations in the microenvironment. When the cancer tumor grows, it encourages angiogenesis, which generates the vascular system, to prematurely develop and provide it with more nutrients. This massive angiogenesis causes irregular and increased amount of interstitial fluid, resulting in a leaky tumor vasculature [82]. Additionally, the development of new lymphatic vessels, as known as lymphangiogenesis [83, 84], is minor and does not match the massive blood vessel development provided by angiogenesis [85]. Although these lymphatic vessels are changed to become larger and more permeable to be able to absorb the increased interstitial fluid [83, 84, 86, 87], they still do not have enough drainage capacity. This results in elevated tumor interstitial fluid pressure (IFP) which drives an interstitial flow out of the tumor and draining into the lymphatic system (Figure 1.3).

The interstitial flow velocity can be measured in vivo with Fluorescent Recovery After Photobleaching (FRAP) or Magnetic Resonance Imaging (MRI). These achievements help scientists quantify the flow. By using FRAP, Chary and Jain [88] found out that in normal tissues and organs, interstitial flow velocity ranged from 0.1 to $2.0 \mu \mathrm{m} \mathrm{s}^{-1}$; later studies have even revealed that the flow can even reach $4.0 \mu \mathrm{m} \mathrm{s}^{-1}$ [89]. Interstitial flow was proven to be elevated in the tumor [90]; the mathematical models also showed that the flow will be predominantly higher at the periphery than at the center of the tumor [91]. This elevated interstitial flow is due to the elevated tumor interstitial fluid pressure caused by the rapid and abnormal angiogenesis process, the peri-tumoral lymphangiogenesis process and the changes in the tumor-associated stroma [92-94]. 


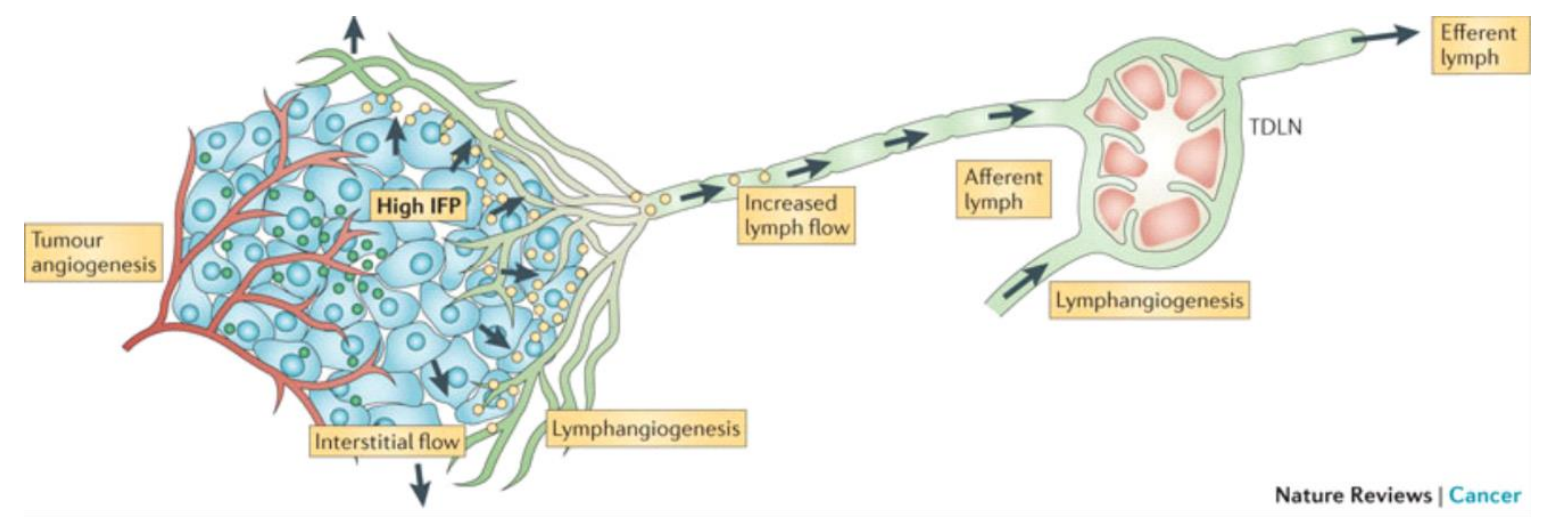

Figure 1.3 Fluid pathways in the tumor microenvironment. Adapted by permission from Macmillan Publishers Ltd: Nature Reviews Cancer, Swartz et al. [95], Copyright 2012.

Given its importance in early metastasis development, we need to find out what the effects of interstitial flow on the tumor development and tumor cell migration are. As reported to have a very slow velocity (from 0.1 to $2 \mu \mathrm{m} \mathrm{s}^{-1}$ ), interstitial flow exerts relatively low shear stresses on the tumor cells, which are covered by the ECM fibers. Therefore, it is reasonable to assume that convection and diffusion, rather than shear, have dominant effects in interstitial flow [82, 95, 96]. Convection and diffusion can transport solutes and create biochemical gradients to direct cell behaviors, such as the autologous chemotaxis mechanism $[75,76]$. In autologous chemotaxis, proteins secreted by the cells diffuse and convect in the flow around the cell. Then, the cell can use membrane receptors to sense the gradient of proteins created by the flow, and thus determine what direction downstream is. For example, due to flow convection of the proteins secreted by the cell, protein concentration will be larger downstream of the cell than upstream of it. The cell is able to sense such concentration differences and follow them $[44,76]$. A specific example of this mechanism is for exampled described by Shields et al. [76]. They suggested that, lymphatic vessels secrete the CCL21 chemokine that attracts tumor cells; while the tumor cells both express the receptor CCR7 and secrete its ligands CCL21. As interstitial flow resulted from the tumor and drains into lymphatic system, the flow forms local gradients of CCL21 under its direction. CCL21 from 
lymphatic vessels will diffuse to the tumor location while tumor cells move towards lymphatic vessels for a higher concentration of CCL21. This could explain how the tumor cells are guided to the lymphatic system (Figure 1.4).

We note that interstitial flow still can generate significant mechanical effects based on shear on the small pores of the tumor and on the extracellular matrix. Indeed, the small pore sizes in the tumoral environment and the high ECM density can induce a local increase of shear stress $[82,95]$. As a result, the flow can for example indirectly affect the tumor cells through the application of drag forces to the surrounding matrix, which stiffens the matrix and promotes the upstream migration of the tumor cells [44].

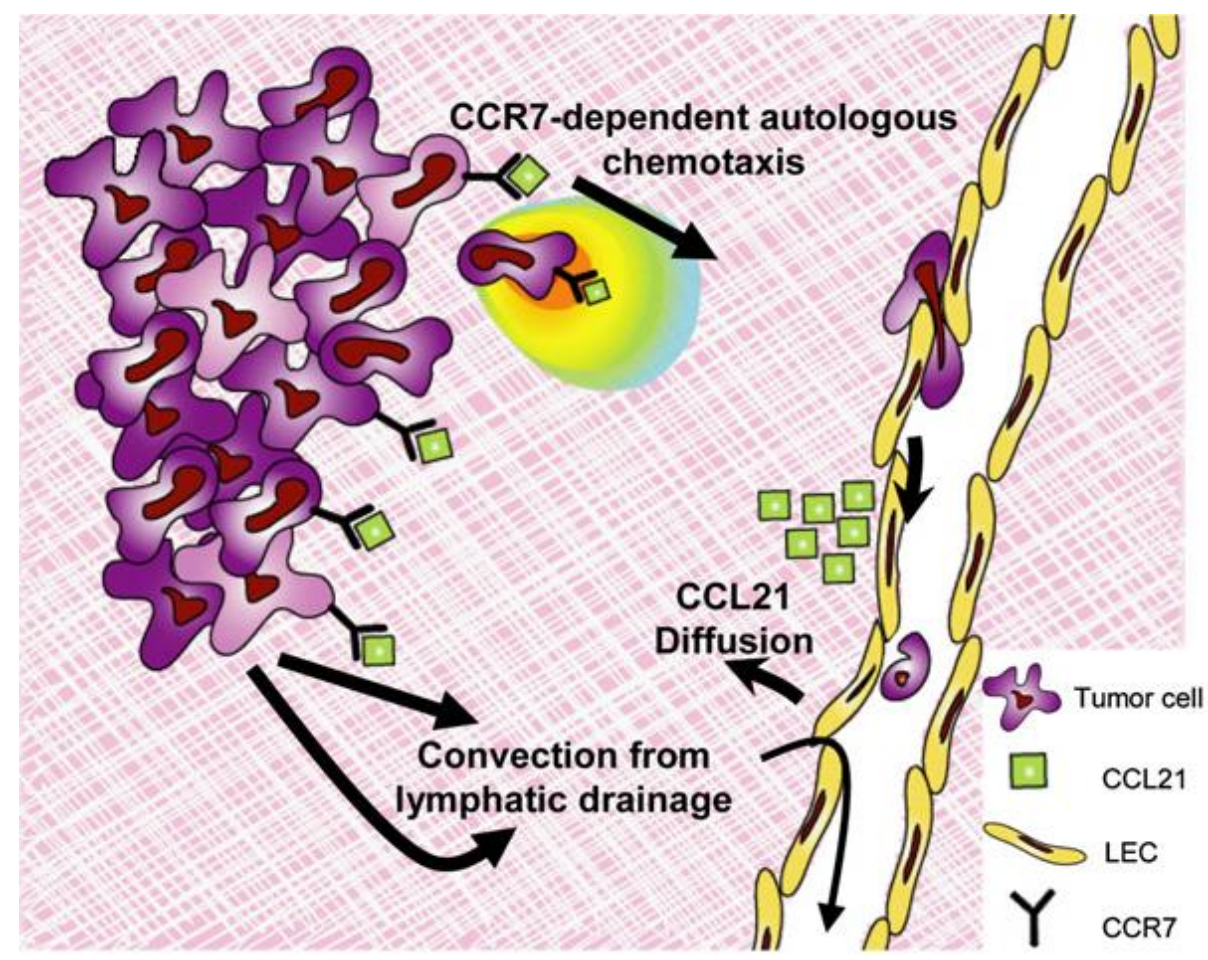

Figure 1.4 The schematic describes the autologous chemotaxis mechanism in tumor microenvironment when interstitial flow creates the CCL21 chemokine gradients guiding the tumor cells to lymphatic vessels. Adapted from Cancer Cell, Shields et al. [76], Copyright 2007, with permission from Elsevier.

The struggle between direct and indirect effects of interstitial flow on tumor progression results in different cell migration behaviors, which can vary depending on 
local flow characteristics and on interactions between cells with each other and with the tumor microenvironment.

In our studies on this topic, our goal is to characterize interstitial flow through a model tumor using a microfluidic platform to investigate the effects of interstitial flow on tumor and how the tumor behaves under multiple flow conditions. 


\section{CHAPTER 2}

\section{Literature review}

This chapter discusses previous research studies in the applications of microfluidics on circulating tumor cell isolation and interstitial flow manipulation.

At the first part of this chapter, some techniques and methods used for cell trapping and isolating are reviewed. Then, we present our research objectives of developing an isolating device that can capture single circulating tumor cells with high efficiency and contribute to metastasis detection, rare cell isolation and cell monitoring.

The second part presents a review of previous studies of interstitial flow. We introduce several designs of microfluidics that we have devised to study interstitial flow through a tumor. We also discuss the fabrication and characteristics of cellular aggregates, which are used in our experiments as a model of a cancer tumor. 


\subsection{Circulating tumor cell isolation}

\subsubsection{Previous studies using microfluidics on CTC isolation}

Microfluidics has been proven to be ideal for achieving a wide range of biological/clinical applications [97]. In particular, isolation and detection of floating cells in liquid biopsy [69] and the following single-cell analysis are made possible with the advancement in microfluidics technology. The main concerns in single-cell isolation are in obtaining a high capture efficiency and high-level of statistical confidence. Low cell capture efficiency warrants a large sample volume, which in turn increases the sample processing time. On the other hand, increasing the flow rate induces higher shear stress, which adversely affects the structural integrity of the cell membrane and significantly impacts the cell viability [98]. For instance, detection of rare cells like CTCs is very challenging due to its low abundance nature [70], in the order of $\sim 1-100$ circulating tumor cells (CTCs) per $10^{9}$ blood cells [71]. Therefore, the primary focus of this research is on the realization of a rapid, sensitive, robust, and microfluidics single-cell isolation device.

Over the past decades, there are many microfluidic methods developed to sort cells based on the dissimilarity in cell properties [99], which can be identified by immunochemical markers associated with different fluorescence [100] or magnetic [101] signals, and the interactions with the environment based on the intrinsic biophysical properties [102]. Although the immunochemical methods provide very promising and specific cell identification, the addition of the fluorescence signals would alter cell properties. Further cell manipulation schemes with external forces [103, 104] are often required which induce technical challenges for both the device operation and the system automation. On the other hand, some sorting schemes consider biophysical properties as the natural biomarkers describing cell conditions [70, 105]. Dielectrophoretic cell sorting techniques apply the natural differences in the dielectric constant of cell bodies, yet the 
external electrical field may trigger cell responses and alterations [106]. Many hydrodynamic (size, density, deformability and morphology) techniques and the related theories have also been developed for effective implementation of cell sorting $[99,107]$.

The inertia-based hydrodynamic cell isolation methods, such as the microvortices trapping of cells through a series of expansion reservoirs, have fast processing time. However, the method suffers a low isolation efficiency of approximately $65 \%$ [108]. On the other hand, separation of CTCs from blood with a spiral microchannel where the Dean flow fractionation results in inherent centrifugal forces for the migration of cancer cells achieved a higher cell retrieval efficiency of approximately 85\% [71]. Nevertheless, the fast movement of cells during the inertial trapping process makes it unable to track single cells, which is vital in some rare cell isolation assays. More recently, higher cell capture efficiency of $\sim 90-95 \%$ was achieved with a microfluidic device with conical-shaped microfilter [109]. However, while the cell trapping through an array of microfilter take delight in higher cell isolation efficiency, the method essentially employs a strategy of scouting for the cells via a "big fishing net", and thus require considerable effort in locating the trapped cells in random positions of the vast array of microtrappers [110]. Additionally, acoustic waves can be applied to achieve high-throughput separation of cells with different physical properties including size, density and compressibility combined as an acoustic radiation force pulling the cells aside to collection outlet [111]. It has also been reported that the cell size and deformation under hydrodynamic stretching at an inertial focusing position can be observed by an automated microscope installed with a high-speed image processing system [112]. Altogether, there remains a need for a cell isolation device that is capable of achieving high isolation efficiency, and with easily traceable cell positions. 
a

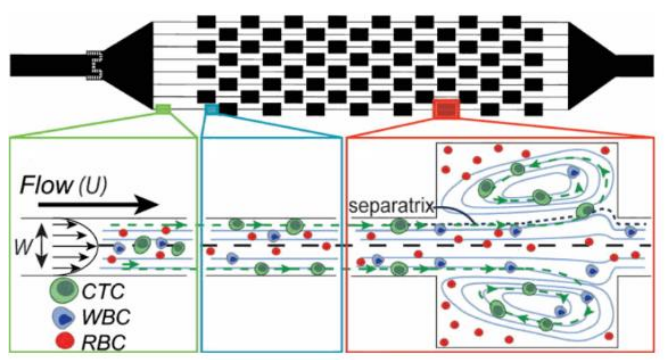

c

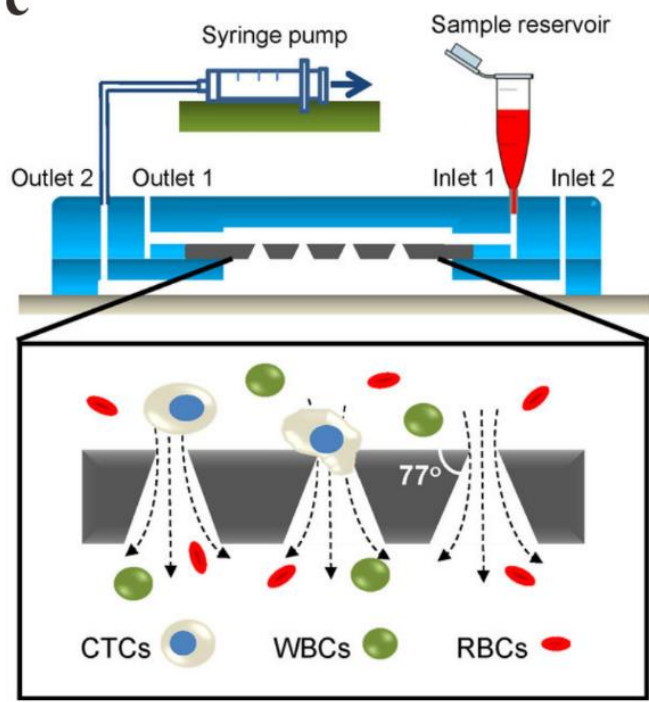

b
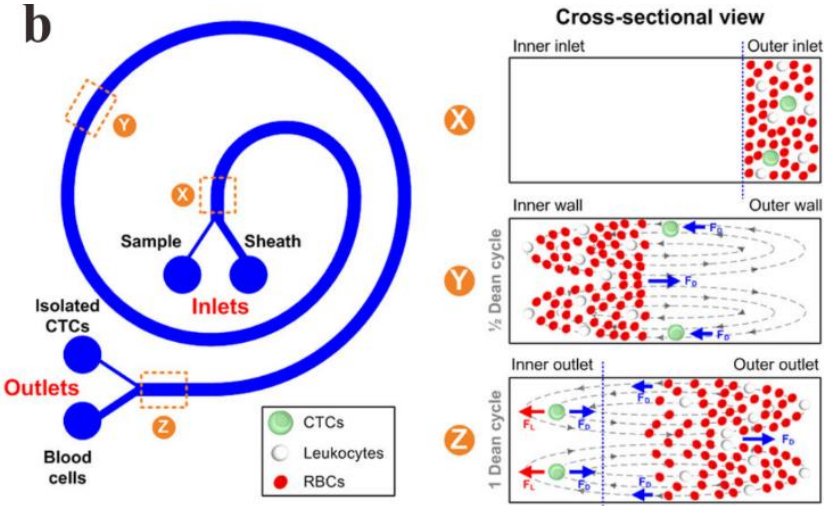

d

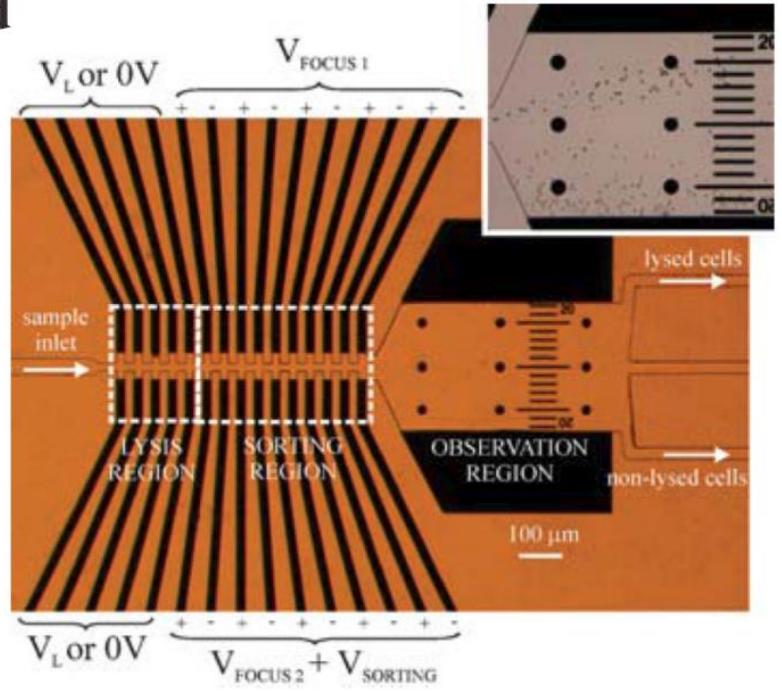

$\mathbf{e}$

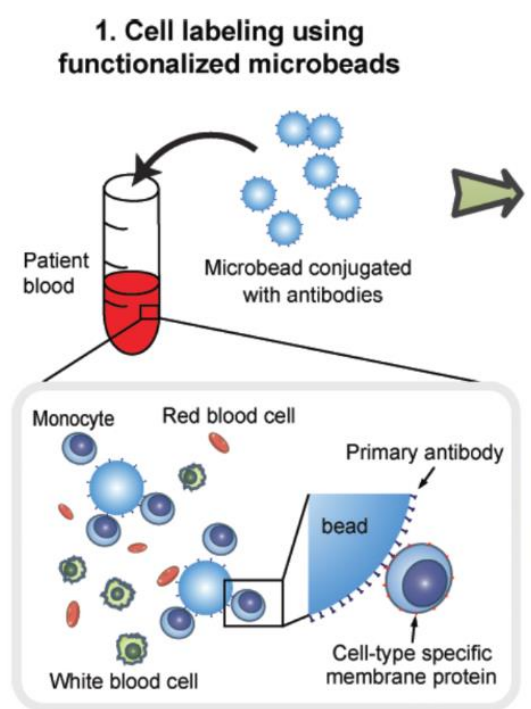

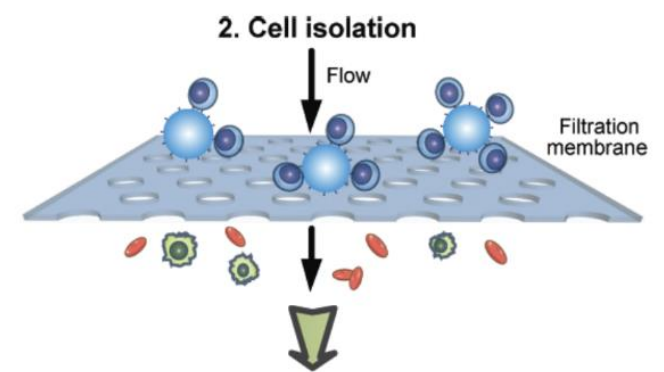

3. Detection of cell-secreted cytokines

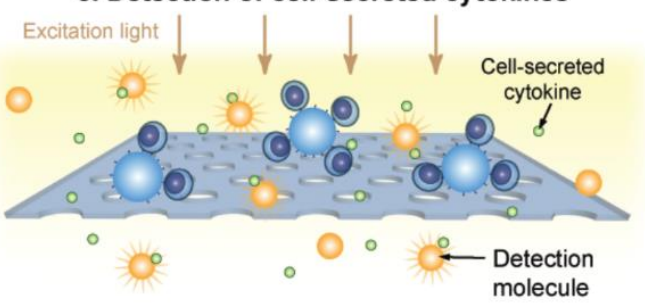

Figure 2.1 Microfluidic designs for cell isolation. (a) Cell isolation method by Dhar et al. [108] using inertia effect in microvortices to trap cells through a series of expansion reservoirs. This method can achieve the isolation efficiency of $\sim 65 \%$. (b) Microfluidic device from Hou et al. [71] separating CTCs from blood with a spiral channel. They achieve a high separating efficiency of $85 \%$. (c) Conical-shaped microfilters from a microfluidic design of Tang et al. [109] can capture cells with high efficiency of $290-95 \%$. (d) Cell sorting technique by Mernier et al. [106] applying dielectrophoresis but it may trigger cell responses and alterations. (e) Cell isolation method by a vast of microtrappers developed by Chen et al. [110]. 
Figure 2.1 (a) reprinted from Biomicrofluidics, Dhar et al. [108], with the permission of AIP Publishing.

Figure 2.1 (b) reprinted from Hou et al. [71], Scientific Reports, 2013.

Figure 2.1 (c) reprinted from Tang et al. [109], Scientific Reports, 2014.

Figure 2.1 (d) reproduced from Mernier et al. [106] with permission from The Royal Society of Chemistry.

Figure 2.1 (e) reproduced from Chen et al. [110] with permission from John Wiley and Sons.

\subsubsection{Research questions}

In this work, we aim to develop a lab-on-a-chip microfluidic device for label-free rapid cell isolation with high efficiency up to $100 \%$. Moreover, the device requires the ability to track the cell positions for further analysis. Therefore, we propose a simple design to isolate single cells in sequence.

As a proof-of-concept for effectiveness of the novel design for cell isolation, we performed a series of parametric study to investigate the trapping characteristics of polystyrene microbeads, and subsequently with a human breast cancer cell line. With the proposed design and framework of the innovative sequential cell isolation device, we are able to precisely isolate floating cells at the pre-determined positions of the device, and thus eliminating the need for exhaustive search and recovery effort required by other physical large-array microstructures trapper schemes for identifying the location of trapped cells.

Our main tasks for this study consist of:

- Come out with the working principle for sequential isolation of cells.

○ Validate the working principle with $80 \mu \mathrm{m}$ microbeads in 'enlarged scale' devices. Since cells are deformable and spheroidal, it will be easier to test the working principle with a simpler model such as microbeads, which are rigid and spherical. 
o Perform parametric tests to achieve the optimal isolation of microbeads in 'enlarged scale' devices.

- Scale down the optimal 'enlarged scale' design to the 'true scale' so that the 'true scale' devices can work with desired cell sizes.

○ Validate the 'true scale' device performance with $20 \mu \mathrm{m}$ microbeads. $20 \mu \mathrm{m}$ microbeads are chosen as they are approximately at the same size as the cells we used in this study.

- Perform the parametric tests to achieve the optimal isolation parameters for cells.

\subsection{Interstitial flow characterization}

\subsubsection{Theoretical model of interstitial flow}

As introduced in 1.4.2, interstitial fluid flow plays an important role in homeostasis and dynamics of living tissues. Interstitial fluid originates from the small portion of fluid that leaks from blood vessels through the endothelium and into intercellular spaces in tissues. Interstitial flow provides tissues with nutrients and evacuates cell waste. However, interstitial flow is also one of the key factors in many cancer progression processes [113]. It promotes the propagation of tumor cell invasion and directs cell migration through the so-called "autologous chemotaxis" mechanism [75]. The low shear stress characteristic of interstitial flow is hypothesized to alter the invasive cancer cell phenotype and enhance its metastatic potential [114]. Interstitial flow also contributes to enhance metastasis progression by activation of fibroblasts and tumor stroma [115]. Nonetheless, due to its ability to transport soluble factors within the tumor environment, interstitial flow is 
indispensable to the delivery of anti-cancerous drugs to tumors. Thus, many studies have recently been carried out to expand our understanding of interstitial flow in tumors.

Interstitial flow can be described mathematically. Considering first interstitial flow in healthy tissue, the fluid flux escaped from blood vessels and into tissue spaces is governed by Starling's equation [116, 117]:

$$
J=L_{P} \frac{S}{V}\left(\left[P_{c}-P_{i}\right]-\sigma\left[\pi_{c}-\pi_{i}\right]\right),
$$

where $J$ is the net fluid flux, $L_{P}$ is the permeability of the blood capillary wall, $S / V$ is the surface area of capillaries of per unit tissue volume, $P_{c}$ is the capillary hydrostatic pressure, $P_{i}$ is the interstitial fluid hydrostatic pressure, $\sigma$ is the reflection coefficient, $\pi_{c}$ is the capillary osmotic pressure and $\pi_{i}$ is the interstitial osmotic pressure. Starling's equation thus states that the fluid flux is driven by both hydrostatic pressure difference between the capillary and the tissue (first term on the left-hand side of Equation (2.1)) and osmotic pressure difference (second term).

The fluid influx into tissue spaces will be mostly returned to the venules, thus preventing an indefinite buildup of interstitial pressure. The difference between capillary influx and venular outflux is absorbed by lymphatic vessels. Between the leakage from capillaries and its capture by venules or lymphatic vessels, interstitial fluid travels through pores of the extracellular protein matrix and interstitial tissue spaces. This fluid transport can thus be mathematically modeled as flow through a porous medium, analogous to water flow through soils or sands. Porous media flow is governed by Darcy's law [118, 119]: 


$$
\tilde{v}=-\frac{K}{\phi \mu} \nabla P
$$

where $\tilde{v}$ is the velocity through pores of the medium or the interstitial velocity, $K$ is the permeability of the tissue, $\phi$ is the porosity of the tissue, $\mu$ is the fluid dynamic viscosity, and $P$ is the interstitial fluid pressure. It is more convenient to rewrite this equation in terms of the so-called Darcy velocity, the average velocity of interstitial flow over a cross section, rather than using the actual velocity in the pores. Darcy velocity or filtration velocity is defined as $v=\tilde{v} \phi$ and can be used to calculate the volumetric flow rate through the porous medium. Therefore, in the case of the one-dimensional flow through a porous medium, equation (2.2) becomes:

$$
v=-\frac{K}{\mu} \frac{\partial P}{\partial x} .
$$

According to this equation, a porous medium is characterized by its permeability, $K$. Experimentally, the permeability can be determined by measuring both the flow velocity and the pressure change through the porous medium, as can be done for example to characterize the permeability of a hydrogel $[52,58]$.

Cancer development brings about many alterations in the tumor microenvironment. When the cancer tumor grows, it encourages massive angiogenesis to provide it with more nutrients. This angiogenesis, together with the lack of lymphangiogenesis, results in an increased fluid influx inside tumor, leading to high overall fluid pressure at the center of the tumor and a significantly decreased pressure at the periphery. This elevated tumor pressure drives an interstitial flow out of the tumor. The mathematical model of Jain et al. [91] describes the distribution of pressure and the interstitial fluid velocity inside a tumor (Figure 2.2a, b). 
Other studies have described porous media flow through a tumor by numerical simulations based on Darcy's law. Numerical simulations provide a visualization and quantification of the distribution of interstitial flow and interstitial fluid pressure in a model tumor (Figure 2.2c, d) [120, 121].

a

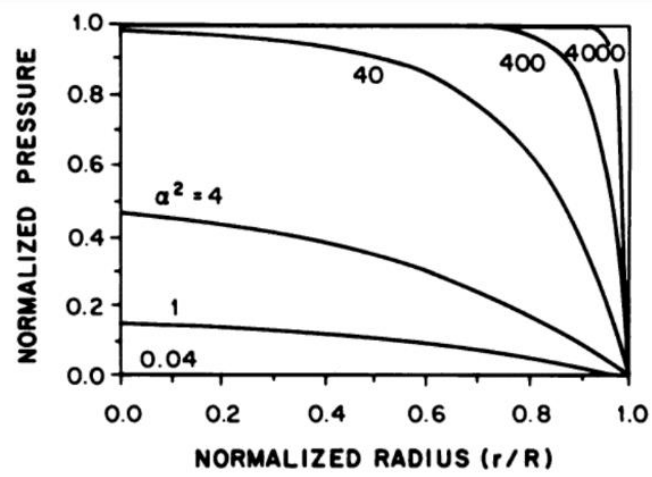

c

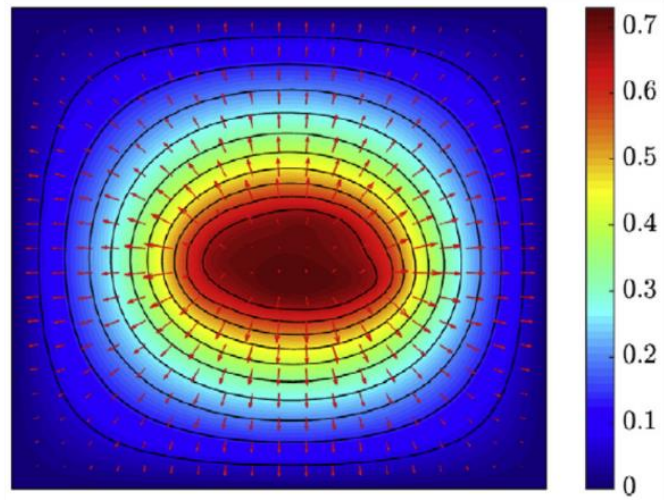

b

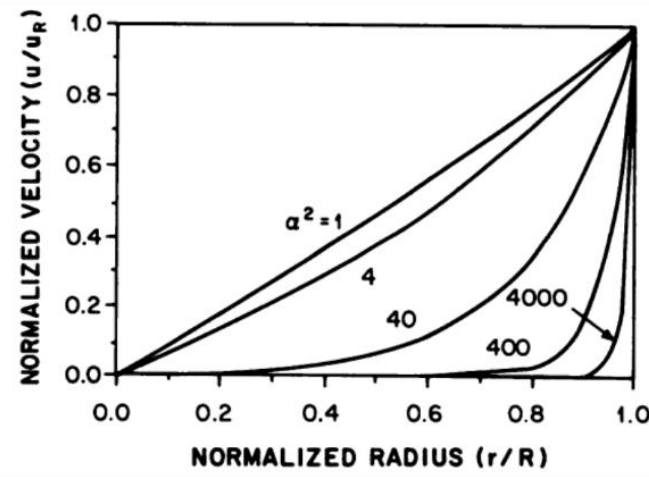

d

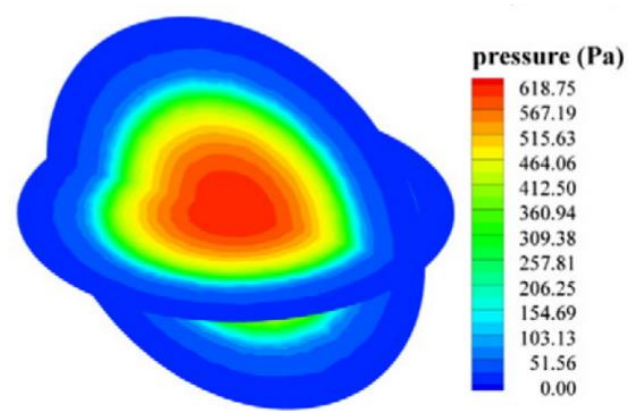

Normal tissue: Spherical Tumor: Spherical $\left(R_{1}=0.75 \mathrm{~mm}\right)$
$V=1.767 \mathrm{~mm}^{3}$ $V^{\prime} / V_{1}=3.913$ $K^{n t} K_{\text {baseline }}=1$

Figure 2.2 Theoretical models to characterize interstitial flow in cancer tumors. (a) The pressure profile and (b) interstitial flow velocity profile inside a tumor, as predicted by the mathematical model of Jain $e$ al. [91]. This model shows that the pressure is highest at the center of the tumor and it drives the interstitial flow out to the tumor periphery. (c) The numerical model by Mohammadi et al. [121] showing the interstitial fluid pressure distribution inside a tumor. Arrows show the direction and magnitude of interstitial fluid flow. (d) 3D numerical model for interstitial fluid pressure contours of the spherical tumor surrounded by a spherical normal tissue conducted by Moghadam et al. [120].

Figure (a), (b) adapted from Cancer Research, Jain et al. [91], with permission from AACR.

Figure (c) reprinted from Microvascular Research, Mohammadi et al. [121], with permission from Elsevier. Figure (d) adapted from Microvascular Research, Moghadam et al. [120], with permission from Elsevier.

Models such as those of Jain et al. and Mohammadi et al. (Figure 2.2) have founded the basis for theoretical studies of interstitial flow through tumors. They have demonstrated that interstitial flow in tumors can be described as flow through a porous medium. These models however use a common simplifying assumption, by describing the 
tumor as an undeformable porous medium of constant permeability. While this assumption allows for a simple model of interstitial flow that can be useful in certain contexts, in this chapter we will show that the mechanical behavior of a tumor mass can significantly differ from a porous medium of constant permeability.

\subsubsection{Microfluidic study on interstitial flow}

In this section, we discuss previous in vitro microfluidic studies to characterize the role of interstitial flow in tumor cell migration. Among many in vitro experimental methods to study cancer migration [38], microfluidics is shown to be the most effective approach in interstitial flow simulation. It is easy to operate fluid flow, precisely control flowrate and pressure, create mechanical and chemical gradients and induce cell-cell signaling while still providing the benefits of live imaging.

Microfluidic assays on interstitial flow have attained significant findings. Roger Kamm's group emerges to be one of the leading groups using microfluidics on metastasis research. They have investigated how interstitial flow influences tumor cell migration through a scaffold of hydrogel. They developed a simple but effective setup where interstitial flow is driven by the hydrostatic pressure difference between two reservoirs placed at the two ends of the hydrogel scaffold (Figure 2.3) [40, 43, 52, 57]. As hydrogel is an inert porous medium, it is feasible to apply Darcy's law to quantify its permeability [58]. Using this setup, they found that interstitial flow promotes the development of threedimensional structures of endothelial cells [58]. Moreover, interstitial flow induces tumor cell migration along flow streamlines, in a manner that depends on cell density, flow velocity and competing autologous chemotaxis [44]. They also investigated the effects of anti-migratory drugs on tumor cell migration under interstitial flow [52]. 
$\mathbf{a}$

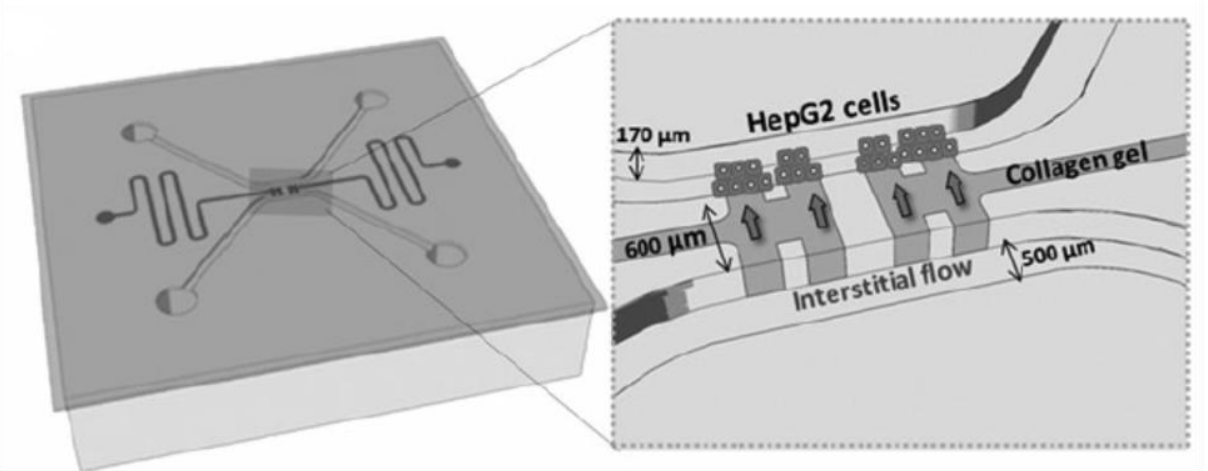

b
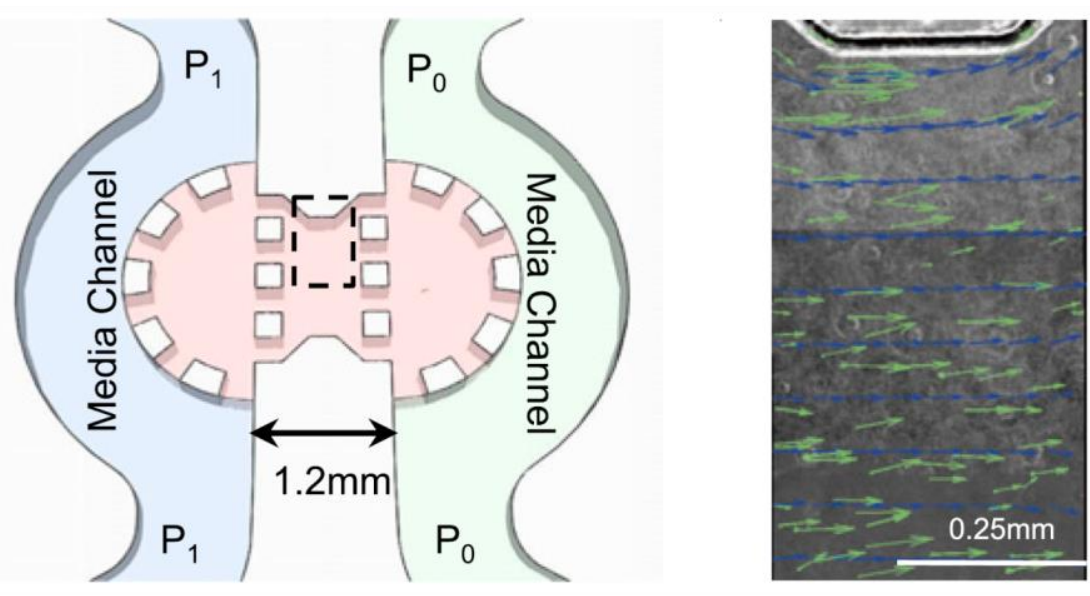

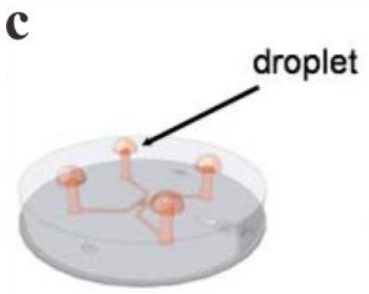

c1

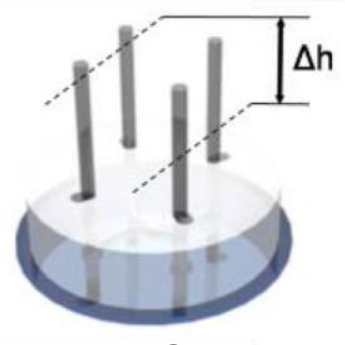

c2

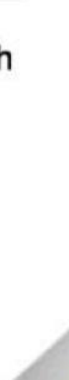

1

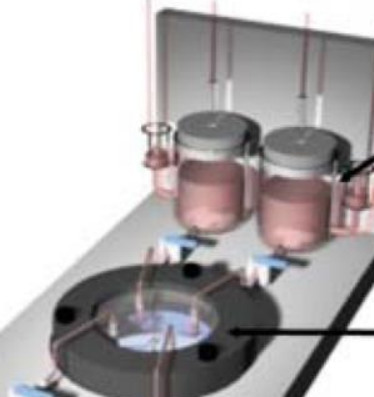

PDMS wafer in
mechanical

clamp

Figure 2.3 Assays from Roger Kamm's group, where they used microfluidics to generate interstitial flow through type I collagen gel scaffold. They characterized interstitial flow through the gel scaffold by using two reservoirs with different levels of medium at two ends of the gel. (a) The gel scaffold design in Kalchman et al. [52]. (b) Microfluidic design by Polacheck et al. on direction of tumor cell migration through a matrix of collagen gel [44]. (c) Flow configurations for static cultures (c1), differential pressure gradient (c2), and generating physiological levels of shear stress (c3) [57].

Figure (a) reprinted from Kalchman et al.[52] with permission of Springer.

Figure (b) adapted from Polacheck et al. [44], Proceedings of the National Academy of Sciences, Copyright 2011, www.pnas.org.

Figure (c) adapted from Vickerman et al. [57] with permission of The Royal Society of Chemistry. 
Melody Swartz's group has developed a microfluidic platform that uses a peristaltic pump to control desired interstitial flow through a variety of flow chambers filled with hydrogels (Figure 2.4a) [45]. They demonstrated that interstitial flow enhances tumor cell migration in $3 \mathrm{D}$ collagen flow chambers by directing the cells to lymphatic vessels. This microfluidic device is claimed to reduce the effort of micro-fabrication in clean room, and the peristaltic pump operation allows a fine tuning of different interstitial flow rates.

a
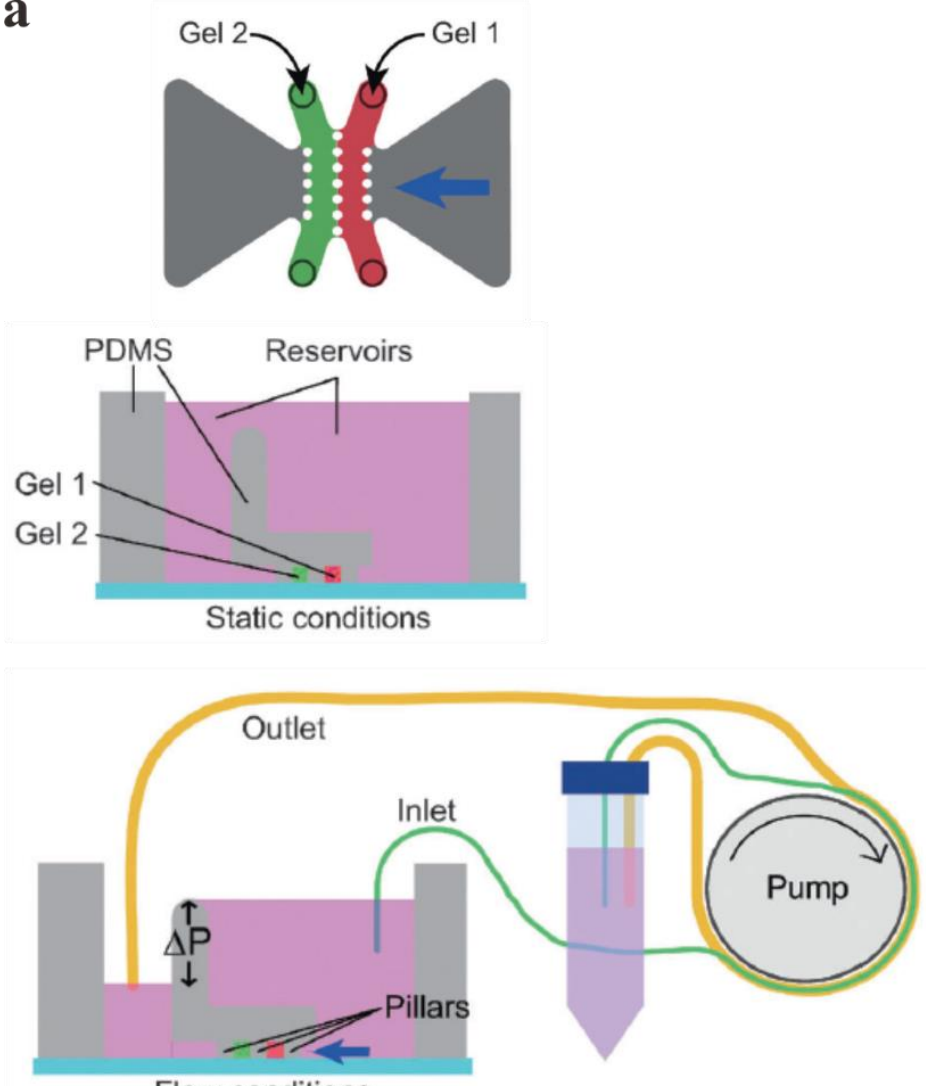

b
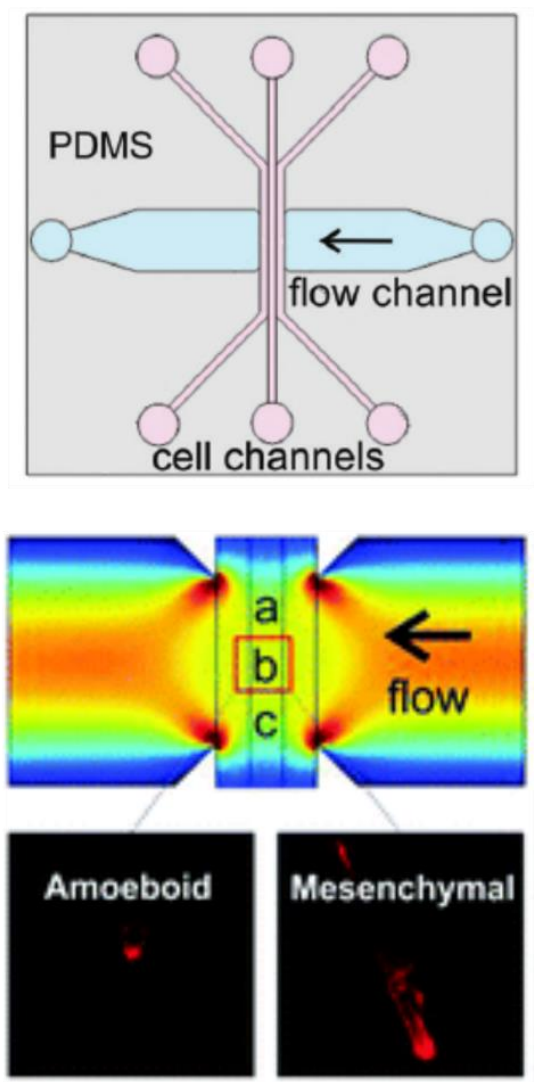

Figure 2.4 The microfluidic device of (a) Melody Swartz's with design of the butterfly-shaped flow chamber [45] and (b) Mingming Wu's group to investigate the major phenotype of cell motility in collagen gel [61].

Figure (a) adapted from Haessler et al. [45] with permission of The Royal Society of Chemistry. Figure (b) adapted from Huang et al. [61] with permission of The Royal Society of Chemistry.

Another remarkable example of application of microfluidics to interstitial flow has been developed by Mingming Wu's group, who uses syringe pumps to apply interstitial flow through tumor cells embedded in collagen gel channels [61]. FRAP method was 
used to measure the flow speed through the collagen channels. Through this experimental model, they have investigated that tumor cells exposed to interstitial flow preferentially migrate by adopting an amoeboid rather than mesenchymal motility phenotype (Figure 2.4b).

The studies discussed above are a few typical examples of microfluidics applications on interstitial flow simulation. This emphasizes the feasibility and flexibility of using microfluidics on interstitial flow research as well as in vitro metastasis assays.

\subsubsection{Research on cellular aggregates}

Following the objectives of the project, a model tumor is required for our study. 3D culture models stand out to be ideal for biomedical research. They overcome several limitations of conventional tissue models in 2D monolayer cultures, such as the lack of interactions between cell vs cell and cell vs extracellular matrix [122-124]. Among 3D culture models, spheroidal cellular aggregates have advantages such as their resemblance to real tissue and the controllable cell-cell adhesion [125].

Cellular aggregates have some specific features that are crucial for in vitro biomedical and biomechanical assays. Studies on transportation of nutrients, waste and therapeutic agents within the cellular aggregate spaces have determined an efficient diffusion distance of 100-200 $\mu \mathrm{m}$, which sets an upper size for cellular aggregate viability [126]. Cellular aggregates with a diameter smaller than about $500 \mu \mathrm{m}$ have been popularly used as models for studies on avascular tissue or tumor mass. By controlling the gene expression of the cells forming the aggregate, previous studies have used cellular aggregates to investigate the role of different genes in tumor behavior [127, 128]. Moreover, cellular aggregates are an ideal model for studies on cell-cell or cell-substrate interactions [129-132]. 
In order to understand the dynamic evolution of a tumor under physiological forcing, a first step is to characterize their material properties. For this reason, previous researchers have investigated the rheology and material behavior of cell aggregates. Recent studies have shown that soft matter models, such as viscoelastic liquids and polymers, can be used to mimic the behaviors of tissues and tumors under forces and gradient pressure. The founding study in this field was performed by Malcolm Steinberg in 1963, who proposed the Differential Adhesion Hypothesis (DAH) as the similarity in physical behavior between embryonic tissues and liquid drops [133]. Liquids consist of molecules with attractive interactions; similarly, tissues consist of cells with adhesive interactions. For this reason, tissues, same as liquids, exhibit a surface tension, which can be measured by micropipette aspiration (Figure 2.5a) $[134,135]$. Also similar to liquid drops on a wettable substrate, cellular aggregates may spread on a substrate depending on the chemical state of its surface tissue, thus displaying an array of wetting behaviors (Figure 2.5c) [136]. More surprisingly, tissue wetting behavior is also sensitive to the rigidity of the substrate [137]. Moreover, tissues exhibit viscoelastic behavior that can be characterized by an elastic modulus and a viscosity [134]. However, there are other physical responses of tissues to force that cannot be described as viscoelastic liquid behavior. For example, under significant forces, cell aggregates fracture like solids. Gonzalez-Rodriguez et al. have characterized the aggregate fracture depending on pulling speed and the intercellular adhesion energy (Figure 2.5b) [138]. These different analogies between the mechanics of tissues and soft matter models have been reviewed by Gonzalez-Rodriguez et al. [139]. 

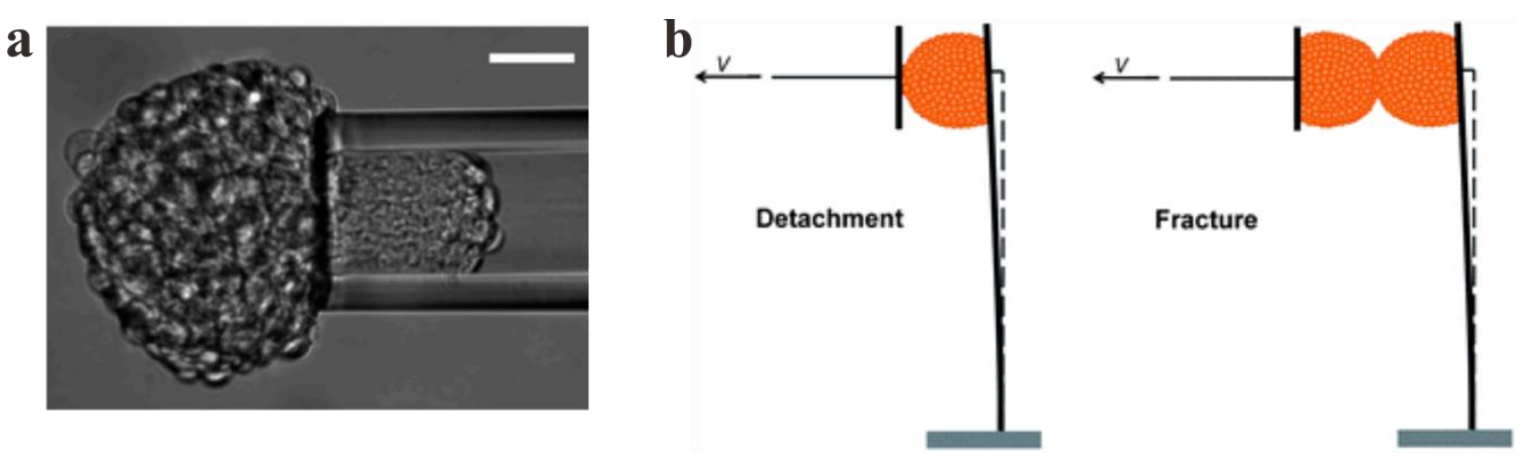

C

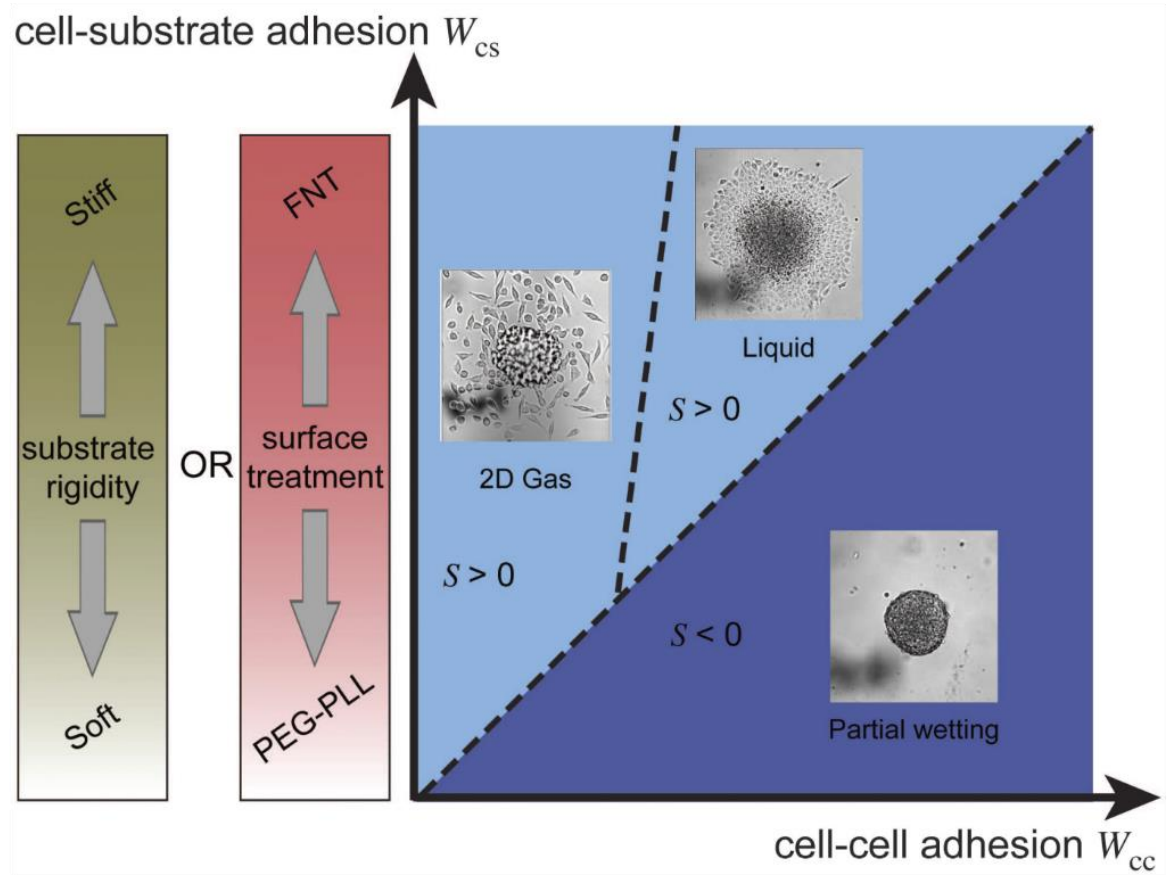

Figure 2.5 Studies on cellular aggregates. (a) Guevorkian et al. [134, 135] measured surface tension of the cellular aggregate by micropipetting aspiration. (b) Gonzalez-Rodriguez et al. investigated aggregate fracture induced by a constant pulling velocity [138]. (c) Tissue spreading assays by Douezan et al. [136, 137] indicate that the wetting behaviors of cellular aggregates are governed either by the competition between cell-cell adhesion vs cell-substrate adhesion or by substrate rigidity. These phenomena demonstrate cellular aggregates behave as both liquids and solids. This figure is introduced from the review of Gonzalez-Rodriguez et al. [139] summarizing tissue mechanical behavior.

Figure (a) adapted from Guevorkian et al. [135], Proceedings of the National Academy of Sciences, Copyright 2011, www.pnas.org..

Figure (b) adapted from Gonzalez-Rodriguez et al. [138] with permission of The Royal Society of Chemistry.

Figure (c) from Gonzalez-Rodriguez et al. [139]. Reprinted with permission from AAAS. 


\subsubsection{Research questions}

As presented in CHAPTER 1 , the combination of interstitial flow in tumors together with other biochemical factors such as tumor cell-cell adhesion changes $[140,141]$ and the differentiation of the extracellular matrix surrounding the tumor [34, 142, 143], simultaneously influences the metastasis progression. Interstitial flow is driven by the high interstitial fluid pressure inside the tumor and drains into the lymphatic system. The physical effects from the high interstitial fluid pressure and the biochemical gradients created by directional interstitial flow help promote the detachment and migration of tumor cells to lymphatic vessels $[76,82]$. The less expression of cell adhesiveness in tumor environment also contribute to the detachment of tumor cells from the primary tumor $[36,140]$. Additionally, interstitial flow can generate significant mechanical effects not only on the small pores of the tumor but on the extracellular matrix as well $[82,95]$. As a result, the flow can indirectly affect the tumor cells through the application of drag forces to the surrounding matrix, which stiffens the matrix and promotes the upstream migration of the tumor cells [44].

Interstitial flow plays an important role in metastasis. Whereas previous studies have focused on understanding the origin of interstitial flow and its role on the migration of cancer cells within the extracellular matrix, little attention has been given to the effects of interstitial flow acting on the tumor mass before cell detachment from the aggregate occurs. Thus, the aim of this study is to develop a microfluidic device to characterize interstitial flow through a tumor, using cellular aggregates as the model tumor. Further understanding the effects of interstitial flow on cancer tumor will provide better insights in the correlation of the physical factors with other biochemical factors happened in tumor environment. 
Our study addresses two central questions:

(1) How to design a microfluidic platform to characterize flow through a cellular aggregate?

(2) How do different flow conditions influence the cellular aggregate?

Upon answering these questions, the specific goals for this study are:

○ Develop of a microfluidic setup to generate different interstitial flow settings,

$\circ$ Design the setup to measure flow rates and pressure differences,

- Introduce cellular aggregates in the device, assuring cell adhesion to the walls and cell viability over time,

$\circ$ Use the setup to characterize flow through a cellular aggregate,

- Study the two-way interactions between flow and the physical response of the cell aggregate. 


\section{CHAPTER 3 Methodology}

This chapter presents the microfluidic design and fabrication for our devices in this project. The ideas and working principles are discussed in detail at the beginning of the chapter.

The device fabrication section shows the detailed procedures for each fabrication technique. We introduce two micro-fabrication techniques: 'Deep Reactive Ion Etching' for CTC isolation devices due to high aspect ratio patterns and 'Photolithography with SU-8' for interstitial flow manipulation devices.

The relevant procedures such as surface treatment, cell culture are also introduced. 


\subsection{Microfluidic design}

\subsubsection{Flow manipulation device for CTC isolation}

The microfluidic device for sequential isolation of floating cancer cells comprises of a series of microsieves positioned at defined locations along the continuous flow stream as described in Figure 3.1a. The novel arrangement of the microsieves enables the trapping of the floating cells in a deterministic and sequential manner. The sample solution is injected to the center inlet, while the buffer flows into the microchannel through the sidechannel inlet, which splits into two streams and sandwiches the sample flow as they progress downstream. As such, the cells would be focused along the axial center of the microchannel. The width of the flow coming out from the center inlet can be accurately controlled by adjusting the flowrate ratio between the two inlets [144, 145]. For example, if the side inlet flowrate is $Q_{s}$ and the center inlet flowrate is $Q_{c}$, due to mass conservation, the width of the center flow would occupy $Q_{c} /\left(Q_{s}+Q_{c}\right)$ of the total channel width. Thus, the cells in the sample solution will be hydrodynamically focused towards the axial center of the microchannel if $Q_{s} \gg Q_{c}$.

In low Reynolds number world (Stokes flow), inertial effect is negligible and the flow field in the microchannel is well defined [146, 147]. When a cell enters the microchannel, its trajectory follows the streamline under laminar regime $[148,149]$ and encounters the array of microsieves. Each microsieve comprises of a semicircular-arc structure with an opening at its center located along the flow channel, with precisely defined lateral offsets (along $y$-direction) and separation distance (along $x$-direction) between the microsieves. The cell, with physical dimension larger than the opening of the microsieve, will be trapped at the microsieve if the streamline passes through the opening. The geometry of the microsieve structures can be optimized for isolation of cells within a workable range of diameter. 
$\mathbf{a}$

Outlet

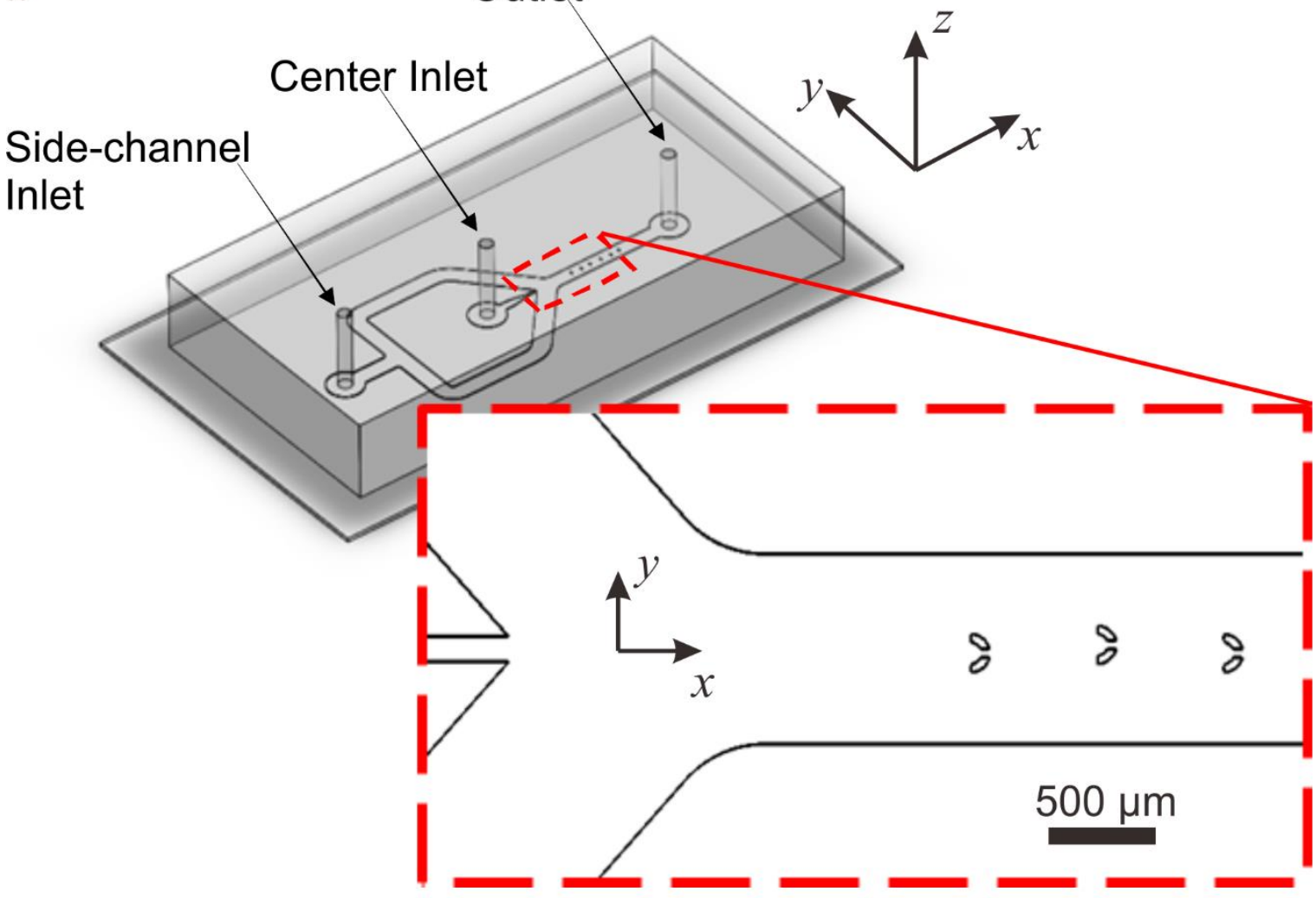

b

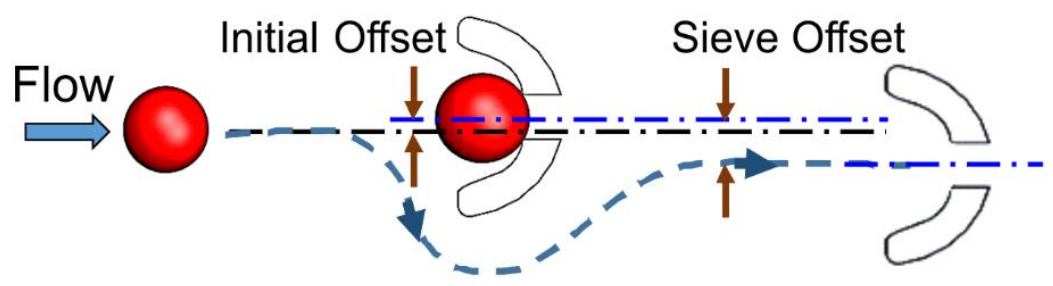

Microsieve \#1

Microsieve \#2

c

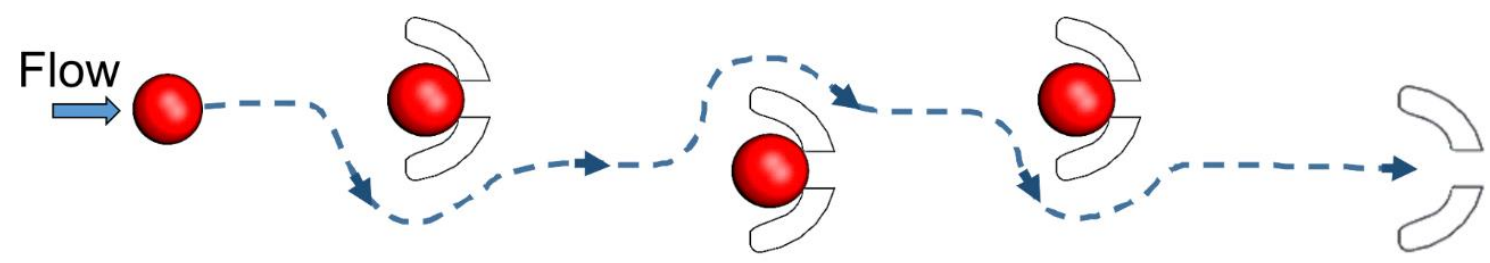

Figure 3.1 (a) Design of the sequential isolation microdevice for floating cells. (b) Configuration of the microsieve positions. With the lateral 'initial offset' from the center of the flow channel, the first floating cell be trapped in the first sieve; and the second cell would predominantly bypass through the negative $y$ side of the first sieve and be trapped at the second sieve position with a lateral 'sieve offset'. (c) The working principle of sequential cell trapping. Adapted from Tran et al. [56] with permission of The Royal Society of Chemistry.

In order to preferentially deflect the trajectory of the subsequent cells after the first microsieve is filled, we introduce a lateral 'initial offset' from the center of the 
microchannel in the positive $y$-direction (Figure 3.1b). The 'initial offset' should be small enough such that the first cell's streamline would still pass through the sieve opening and the cell would be trapped in the first microsieve. The second incoming cell would bypass through the negative $y$-direction of the first sieve due to the aforementioned offset. Subsequently, we introduce another lateral offset, known as the 'sieve offset' to the second microsieve such that the second incoming cell trajectory passes through the sieve opening of the second sieve for the isolation of the second cell. The 'sieve offset' simply represents the lateral distance between the axis of symmetry of the first and second sieves. Note that, the sieve offset needs to be larger than the initial offset but smaller than the outer radius of the sieve, such that the third incoming cell, after bypassing the second microsieve, will move to the positive $y$-direction and encounter the third microsieve.

The positioning of the third and fourth sieves is a repeat of the first and second sieves (Figure 3.1c). The number of sieves can be increased up to the total number of desired number of cells isolation using the mentioned pattern. In this work, we demonstrate beads and cells sequential isolation with a total of 50 microsieves. The microsieve lateral offsets - 'initial offset' and 'sieve offset' are varied in search for the optimal trapping efficiency. As the flow conditions are well defined at low Reynolds number, the microfluidic device can achieve high-yield, high-repeatability cell isolation at defined positions.

We first validated the sequential cell isolation concept using the 'enlarged' device to demonstrate the isolation of $80 \mu \mathrm{m}$ diameter polystyrene microbeads. The device consists of 50 identical microsieves (inner diameter: $100 \mu \mathrm{m}$; outer diameter: $140 \mu \mathrm{m}$; gap width: $40 \mu \mathrm{m}$ ) located along the flow channel (width: $900 \mu \mathrm{m}$; thickness: $120 \mu \mathrm{m}$ ) of the device. Subsequently, the dimension of the microfluidic device is scaled down by a factor 
of four to establish the 'true-scale' isolation device with a sieve opening of $10 \mu \mathrm{m}$ for isolation of $20 \mu \mathrm{m}$ diameter polystyrene microbeads and cancer cells.

\subsubsection{Interstitial flow manipulation device}

In tissue or tumor environment, interstitial flow starts from blood vessels covering one side of the tissue/tumor and drains into lymphatic vessels at the other side of the tissue/tumor (as described in Figure 1.3). We first chose cellular aggregates as the model system to represent a tumor. To characterize the interstitial flow through a tumor in a microfluidic device, our design idea is creating a chamber to contain the cellular aggregate and a channel going through the chamber to apply flow through. The channel at the one side of the chamber plays as the source of interstitial fluid escaped from the blood vessels. The channel at the other side of the chamber acts as the sink for the fluid, just like lymphatic vessels.

In this study, our initial hypothesis is that the cellular aggregate behaves as a porous medium. In order to investigate the question of how different flow conditions influencing the cellular aggregate, we introduced two designs to characterize two types of flow: 'constant flow rate' and 'continuously increasing flow rate'.

\section{First design of microfluidic device for interstitial flow with constant flow rates}

This device includes 5 microchannels connected to a central chamber in square shape with dimensions of $500 \times 500 \mu \mathrm{m}$. (Figure 3.2). This chamber is the place that contains and holds a cellular aggregate through which flow will be applied. We also have an alternative design for the chamber that includes pillars to hold the contents of the chamber, which can be used for hydrogel assays (see inset in Figure 3.2). Channels 1 and 5 are, respectively, the input and output channels for the main flow through the chamber. These channels have a width of $300 \mu \mathrm{m}$ for the cellular aggregate version, and $400 \mu \mathrm{m}$ for 
hydrogel version. Channel 3 is the path to introduce a cellular aggregate into the chamber, previous to the start of the flow experiment. The width of channel 3 should be chosen so that the cellular aggregate can easily recover its shape once arrived at the central chamber. Thus, the width of channel 3 is set to $300 \mu \mathrm{m}$. Channels 2 and 4 are $100 \mu \mathrm{m}$ wide and are used for measuring the pressure drop across the chamber while the flow is applied. These two channels connect the two ends of the chamber to two digital manometers to monitor the pressure difference across the chamber in real time.

For this device, different constant flow rates are applied from the channel 1 through the cell chamber. We can acquire the flow rate through the aggregate and measure the pressure drop across the aggregate. If the aggregate behaves as a simple porous medium, we can then apply Darcy's law to estimate the aggregate's permeability.
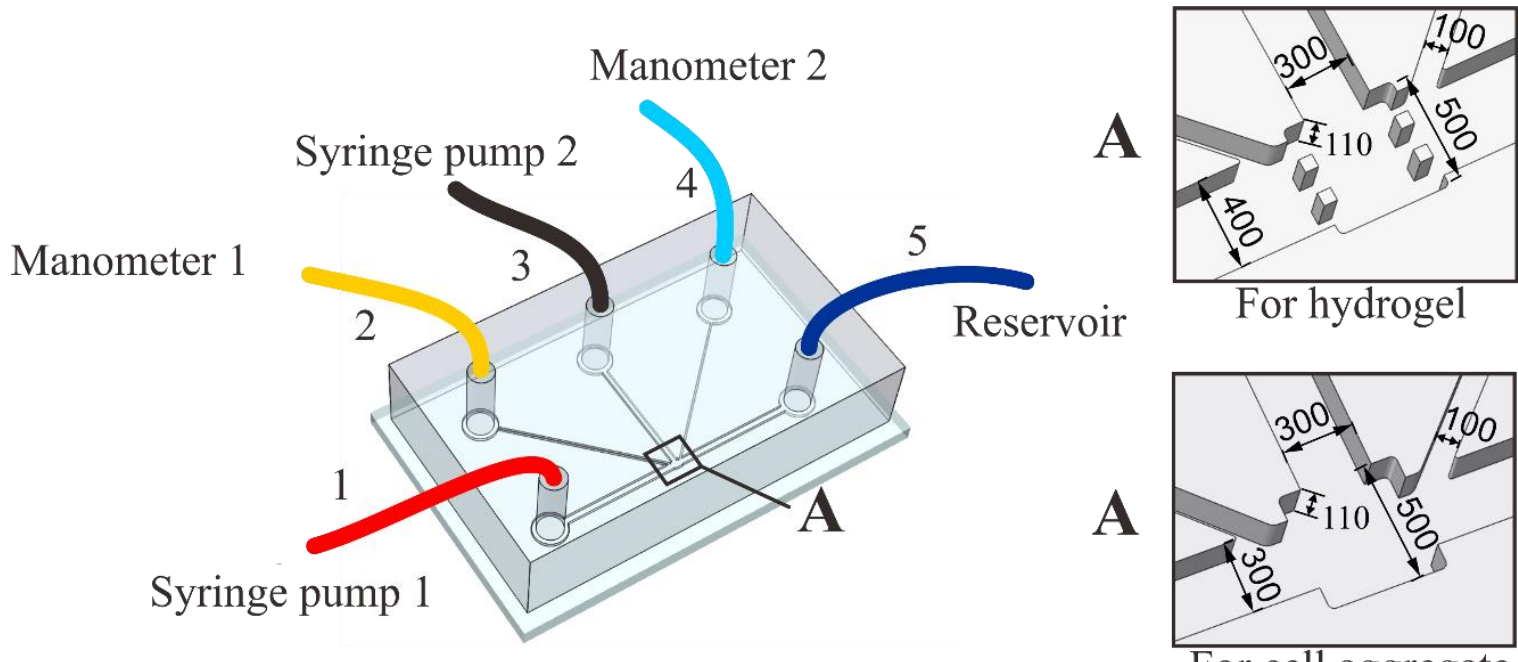

For hydrogel

A

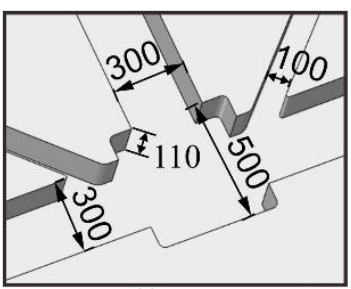

For cell aggregate

Figure 3.2 Microfluidic design to characterize interstitial flow with constant flow rates. The device consists of five microchannels connecting to a central chamber. There are two designs for the chamber: chamber with pillars for hydrogel assays (top right) and without pillars for cellular aggregate assays (bottom right). The device is connected with two syringe pumps and two manometers during experiments. Dimensions are in $\mu \mathrm{m}$.

Physiologically, blood flow is brought to the core of a tumor by angiogenic vessels, then it leaks through the tumor, and finally it is collected by the lymphatic vasculature. Our microfluidic model intends to represent the leaked flow from the high- 
pressure region inside the tumor, to the outside of the tumor. From a biological perspective, our microfluidic model must not be interpreted literally as flow crossing a tumor, but rather as a model of the radially outgoing flow from the tumor core (represented by the high-pressure side of the microchannel) to the outside (the lowpressure side). The cellular aggregate is thus not strictly speaking a representation of the whole tumor, but rather of a rather section from its core to its periphery.

\section{Second design of microfluidic device for interstitial flow with continuously increasing flow rate}

The design for continuously increasing flow rate is modified from the constant flow rate design. This design consists of the cell chamber connected to the input, output and cell aggregate entrance microchannels, same as in the previous design. The modification in this new design is the addition of a thin side channel in parallel with the cell chamber, with the desired hydrodynamic resistance to the flow (Figure 3.3a).

$\mathbf{a}$

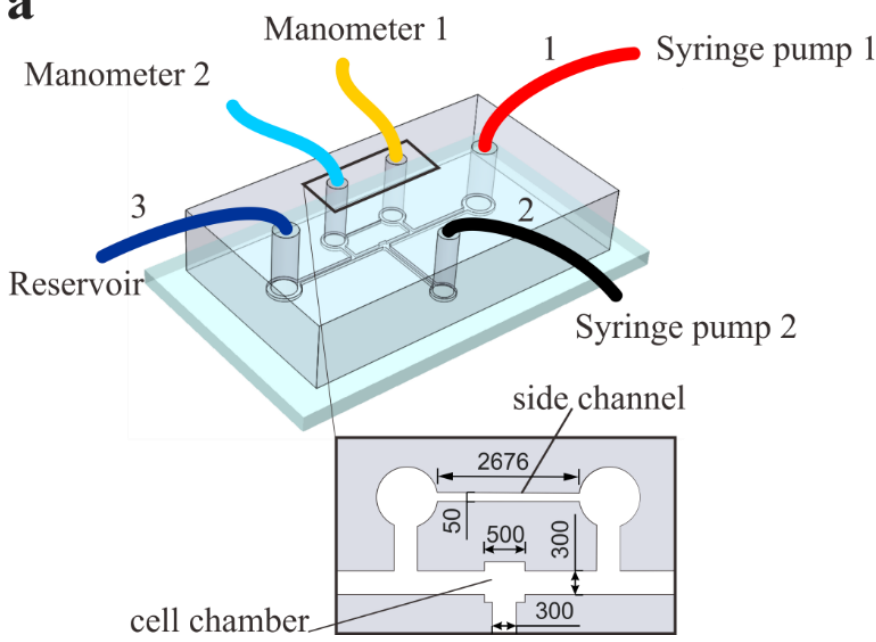

b

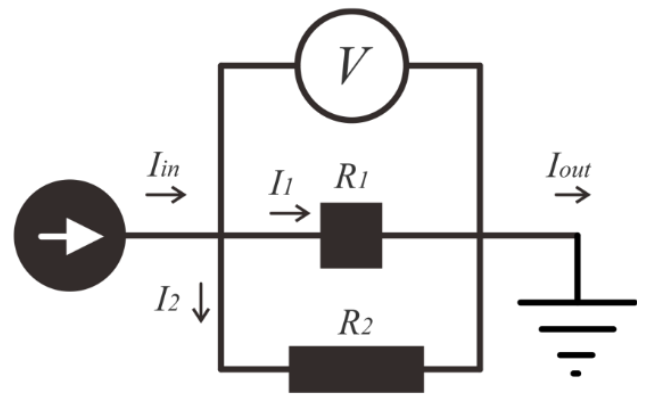

Figure 3.3 (a) Schematics of the second design with the side channel in parallel with the cell chamber. Dimensions are in $\mu \mathrm{m}$. (b) The equivalent electric circuit to the second design. Here, $R_{I}$ is the resistance of the cell chamber, $R_{2}$ is the resistance of the side channel. Being parallel to each other, the resistances receive the same voltage, and similarly the channels receive the same pressure difference. 
When applying a flow rate to the input channel, a large portion of the input flow preferentially moves to the side channel, which has been designed to have a lower hydraulic resistance than the cell aggregate. This results in a pressure difference at two ends of the side channel. Due to the parallel-channel design, this pressure difference across the side channel is equal to the pressure difference across the cell chamber. Since the side channel can be calibrated and its hydraulic resistance determined, the pressure difference can be determined from the flow rate going through the side channel.

The ratio of flow rate going through the cell chamber and the side channel is the inverse of the ratio between the hydraulic resistance of the side channel and the resistance of the cell aggregate inside the chamber. For this reason, we first need to measure the hydrodynamic resistance of the side channel, so that it can then be used to measure the pressure difference across the cell chamber, thus replacing the manometer.

This design is analogous to an electrical circuit with two parallel resistances (Figure 3.3b). In such a circuit, the voltage applied to both resistances is the same. If resistance $R_{1}$ is much higher than resistance $R_{2}$, the current will mostly go through $R_{2}$, whereas only a small current will go through $R_{l}$. The voltage can be flexibly varied by the current through $R_{2}$. Applying these ideas to our second design, we have a microfluidic device which can apply a slow flow rate through a cell chamber while the pressure difference can respond very fast with a small change of the flow.

We keep the thickness of all microchannels at $\sim 100-110 \mu \mathrm{m}$, same as in the first design. The size of the chamber remains at $500 \times 500 \mu \mathrm{m}$. Channels 1,2 and 3 also remain at a width of $300 \mu \mathrm{m}$. The side channel is $50 \mu \mathrm{m}$ wide, $2.676 \mathrm{~mm}$ long and was designed to have a pre-defined hydrodynamic resistance to water at $30 \mathrm{~Pa}^{\mathrm{min}} \mu \mathrm{l}^{-1}$. The hydrodynamic resistance is defined as: 


$$
\Delta P=Q \times R_{h},
$$

where $\Delta P$ is the pressure drop across the channel and $Q$ is the flow rate through the channel.

For a microchannel with rectangular cross-section, the channel resistance can be calculated as:

$$
R_{h} \approx \frac{12 \mu L}{w h^{3}(1-0.63 h / w)}(\text { with } h<w)
$$

where $R_{h}$ is the hydrodynamic resistance of the microchannel, $\mu$ is the viscosity of the fluid flow, the channel with height $h$ and width $w, L$ is the length of the channel.

The side channel resistance is designed to be $30 \mathrm{~Pa} \mathrm{~min} \mu \mathrm{l}^{-1}$ as we want to achieve a pressure difference of $30 \mathrm{~Pa}$ when applying an input flow rate of $1 \mu 1 \mathrm{~min}^{-1}$.

The main downside of this design is that we cannot simultaneously measure pressure difference and flow rate going through the cell aggregate. Indeed, the applied flow rate is mainly diverted through the side channel, thus rendering the determination of the flow rate through the cell aggregate very imprecise. This design cannot thus be used to estimate aggregate permeability. However, the fast response of pressure value following the small changes of flow rates helps us constantly observe the pressure difference value across the cell chamber under different loading rates of the flow. 


\subsection{Device fabrication}

\subsubsection{Fabrication of the master mold}

In this step, we used micro-fabrication techniques to produce the master containing our design patterns. We fabricated the master for CTC isolation devices using Deep Reactive Ion Etching method and Photolithography with SU-8 for interstitial flow manipulation devices.

\section{Micro-fabrication using Deep Reactive Ion Etching}

For the CTC isolation devices, the master mold is fabricated with Deep Reactive Ion Etching (DRIE). DRIE is necessary because of the high aspect ratio of the microsieve structures. The aspect ratio between the width of the microsieve and its thickness is 1:6, which is unable to fabricate by the standard photo-lithography method. We transferred the design of the microfluidic sequential microsieves drawn with AutoCAD (Autodesk, USA) to a mask for microfabrication of a negative silicon mold. For the 'enlarged' devices, we used a plastic mask with printing resolution 1/16 inch. For the 'true scale' devices, we had to use a Chrome mask since the smallest micro-structures in the design are smaller than the resolution of the plastic mask.

The detail of fabrication process is as following:

1) Deposit a 5- $\mu \mathrm{m}$ thin layer of AZ-9260 positive photoresist onto a silicon wafer substrate to create a photoresist mask for the etching process (Figure 3.4a-c).

- Cleaning a wafer with Acetone and Isopropanol. Then rinse it with distilled water. Use nitrogen blower to dry the wafer surface.

- Place the wafer on a hot plate at $110^{\circ} \mathrm{C}$ in 7 minutes to dry it. 
- Spin coating a layer of AZ-9260 onto the wafer

i. First spin: $500 \mathrm{rpm}$, at $100 \mathrm{rpm} \mathrm{s}^{-1}$ for 10 seconds

ii. Final spin: $3500 \mathrm{rpm}$ at $300 \mathrm{rpm} \mathrm{s}^{-1}$ for 30 seconds

- Softbake the wafer at $110^{\circ} \mathrm{C}$ for 80 seconds. After baking, let the wafer cool down slowly to room temperature.

- Use the chrome mask with designed patterns aligning with the wafer by Mask Aligner machine. Karl Suss Mask Aligner MA6 was used in this fabrication.

- Expose the photoresist under UV. Choose contact type as hard. The exposure dose and exposure time is followed AZ-9260 manual.

- Develop the wafer in 1 minute. We achieve the result as the photoresist covering all the wafer area except the design pattern (Figure 3.4c).

2) Apply DRIE to engrave the microfluidic pattern on the silicon wafer. The process resulted in a negative silicon master with $\sim 110 \mu \mathrm{m}$ deep patterns for the "enlarged scale' devices and $\sim 30 \mu \mathrm{m}$ for the 'true scale' devices (Figure 3.4d).

3) The etched wafer was cleaned with Acetone to remove the AZ-9260 photoresist mask.

4) The DRIE causes high roughness on the etched surfaces. This affects the quality of the next molding process. Therefore, the wafer was surface-treated with oxygen plasma activation (energy: $5 \mathrm{~kJ}$; Harrick plasma cleaner PDC-002) and deposition of a molecular monolayer of trichloro $(1 \mathrm{H}, 1 \mathrm{H}, 2 \mathrm{H}, 2 \mathrm{H}$-perfluoro-octyl)silane (Sigma-Aldrich, St. Louis, MO). This is to facilitate the release of the cross-linked PDMS (Sylgard 184, Dow Chemical, Midland, MI, USA) from the silicon master. 


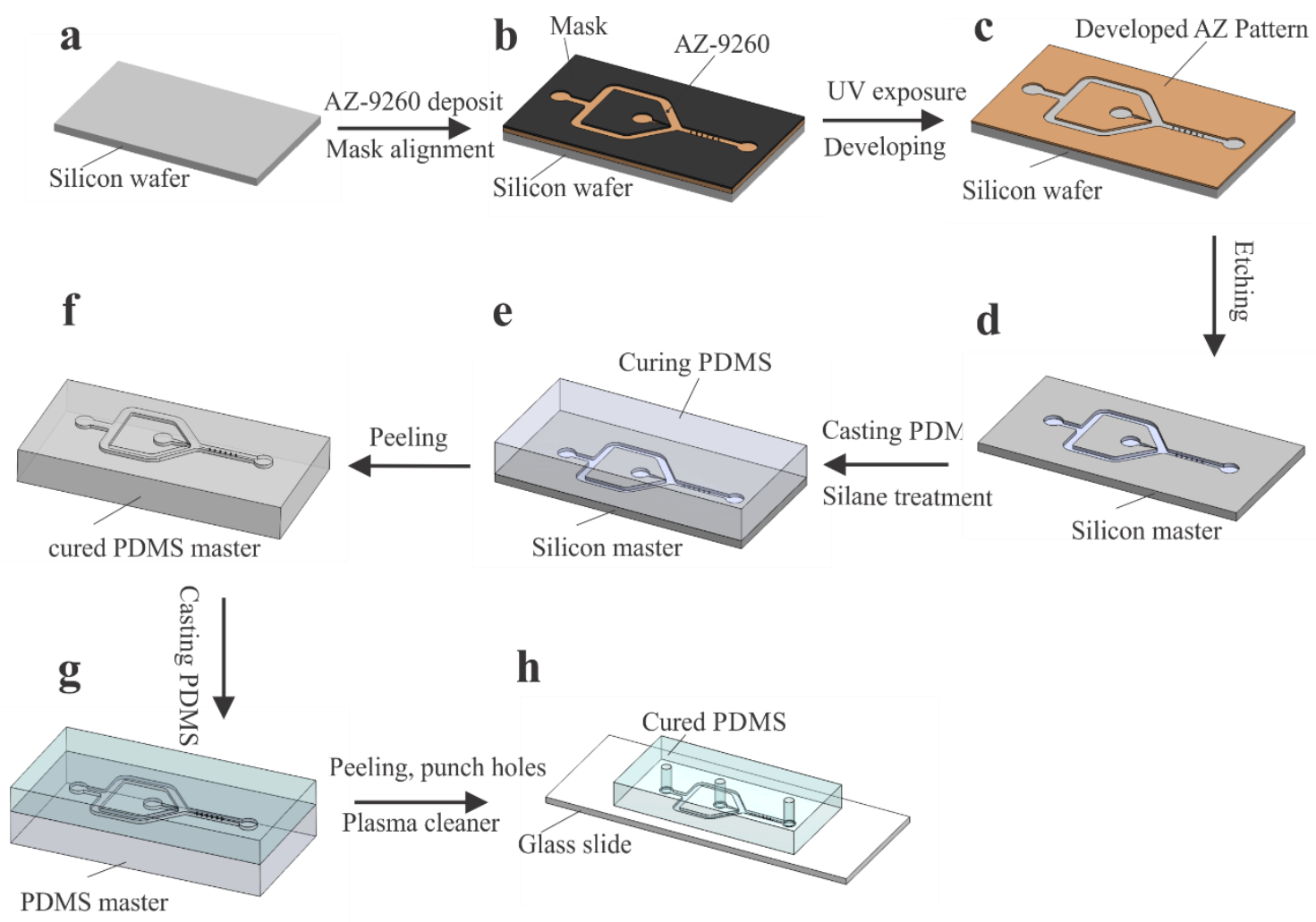

Figure 3.4 Cell isolation device fabrication procedure. (a-c) photolithography with AZ-9260 to deposit a positive photoresist pattern on a wafer. (c-d) Deep-Reactive-Ion-Etching to engrave the designed pattern onto the silicon master. (e-f) Produce the PDMS master by two-stage replica molding. (g-h) Casting PDMS, and plasma bonding with a glass slide to produce the final device.

\section{Photo-lithography fabrication process with SU-8 2100}

For the interstitial flow characterization devices, we did not face the issue of high aspect ratio patterns. Thus, the master mold in this study was fabricated by the standard photolithography method to deposit photoresist patterns onto a silicon wafer. The negative photoresist SU8-2100 (MicroChem, Newton, Massachusetts, USA) was used to achieve a master mold including microchannel patterns with the desired thickness of $\sim 100-110 \mu \mathrm{m}$. 


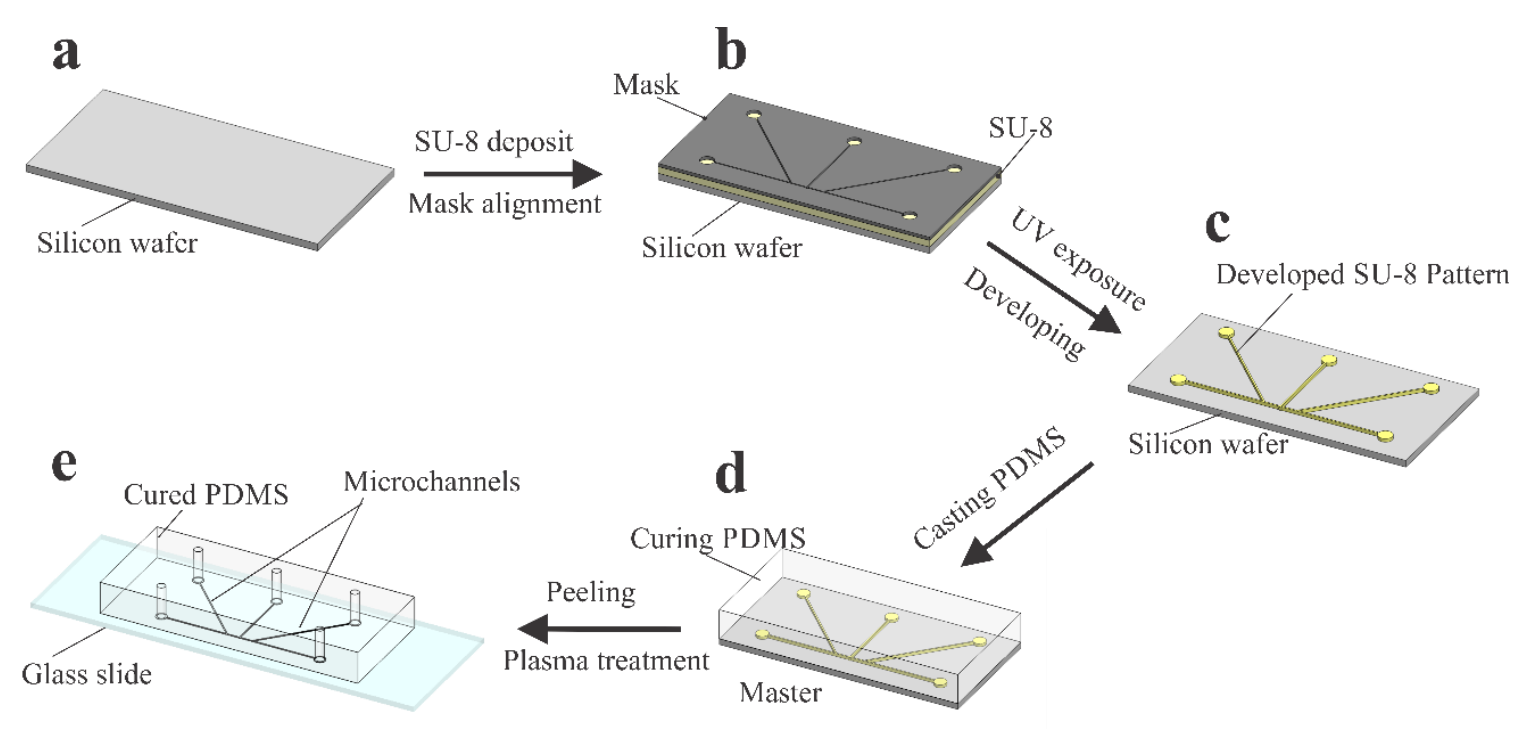

Figure 3.5 Schematics of microfabrication procedure for cellular aggregate microfluidic device. (a-c) Photolithography process with negative photoresist SU-8 2100 to deposit a pattern of $\sim 100-110 \mu \mathrm{m}$ in thickness. (d-e) Soft lithography process with PDMS bonding with a glass slide to form a microfluidic device.

Detail fabrication procedure as following:

1. Cleaning a wafer with Acetone and Isopropanol. Then rinse it with distilled water. Use nitrogen blower to dry the wafer surface.

2. Place the wafer on a hot plate at $110^{\circ} \mathrm{C}$ in 7 minutes.

3. Spin coating a layer of SU-8 onto the wafer

- First spin: $500 \mathrm{rpm}$, at $100 \mathrm{rpm} \mathrm{s}^{-1}$ for 10 seconds

- Final spin: $2000 \mathrm{rpm}$ at $300 \mathrm{rpm} \mathrm{s}^{-1}$ for 30 seconds

* With SU-8 2100, the spin speed is chosen from Microchem manual for SU8-2100 to achieve $100-\mu \mathrm{m}$ thickness.

4. Prebake the wafer at $65^{\circ} \mathrm{C}$ for 10 minutes and then bake at $95{ }^{\circ} \mathrm{C}$ for 25 minutes. After baking, let the wafer cool down slowly to room temperature.

5. Use a transparent mask with designed patterns aligning with the wafer by Mask Aligner machine. Here we use Karl Suss Mask Aligner MA6. 
6. Expose the photoresist under UV. Choose the UV exposure dose based on MicroChem manual with the contact type as hard.

7. After exposure, bake the wafer at $65^{\circ} \mathrm{C}$ in 10 minutes then continue baking at $95^{\circ} \mathrm{C}$ in 15 minutes on the hot plate.

8. Develop the wafer in 10 minutes.

9. Rinse the wafer with Isopropanol and distilled water, and then dry it by nitrogen blower or by wafer dryer machine.

\subsubsection{Fabrication of PDMS chips}

Our devices were fabricated using soft lithography [150, 151]. The master is molded in Polydimethylsiloxane (PDMS; Sylgard 184, Dow Chemical, Midland, MI, USA) to form a 5-mm-thick PDMS chip. The detail procedure as following:

1) Determine the necessary amount of PDMS by estimating the volume of PDMS needed and consider the PDMS density is $965 \mathrm{~kg} \mathrm{~m}^{-3}$.

2) Mix the PDMS monomer with the curing agent at ratio 10:1.

3) Put the blended PDMS inside a degasser, wait until all the bubbles are gone.

4) Place the clean patterned wafer in a petri-dish, and then gently pour the degassed PDMS solution to the petri-dish.

5) Leave the petri-dish inside an oven at $60{ }^{\circ} \mathrm{C}$, the PDMS will be cured in 2-4 hours.

6) Cut the cured PDMS in patterned areas.

7) Punch the holes for the PDMS chips by using Harris Unicore puncher.

8) Soak the PDMS chips into distilled water for 24 hours to deionize them.

Notice: The silicon master for the CTC isolation devices is a 'negative' master mold. Therefore, the PDMS substrate for CTC isolation devices was fabricated by the two-stage replica molding technique using PDMS solution with 10:1 mixture ratio [150, 152-154] 
(Figure 3.4e-f). First, the 'negative' silicon master is molded with PDMS solution to produce the 'positive' PDMS master mold. Then, the 'positive' PDMS master mold will be used to mold the PDMS substrate.

\subsubsection{Device assembly using plasma bonding}

After the PDMS chips are produced, we need to assembly them with substrates. Here the PDMS chips are bonded with glass slides using Plasma Cleaner machine. The procedure is described below.

1) Prepare glass slides, and clean them with acetone, then rinse with isopropanol and distilled water.

2) If the PDMS chips get dirty, we also need to clean them with isopropanol and distilled water.

3) Put the PDMS chips and glass slides in a petri-dish and place inside an oven at $80{ }^{\circ} \mathrm{C}$ for $15-30$ minutes to make them completely dry.

4) We use the Plasma Cleaner (Harrick Plasma PDC-002) for this process. Apply air plasma treatment with power $30 \mathrm{~W}$ for 30 to 45 seconds to activate the surfaces of PDMS and glass slides.

5) Gently place two activated surfaces against each other, and press slightly.

6) Put the bonded device into an oven at $80^{\circ} \mathrm{C}$ in at least 30 minutes to secure the bonding.

\subsection{Surface treatment}

According to different purposes of the experiments, the inner surfaces of the microchannels need to be treated with suitable coating solutions. PDMS and glass surfaces can be coated with various reagents, polymers and extra-cellular matrix proteins. 
Table 3-1 Summary of surface treatment procedures used in the whole project

\begin{tabular}{|c|c|c|c|}
\hline Coating solutions & Experimental objects & Coating conditions & Experiment purposes \\
\hline $\begin{array}{l}6 \% \text { BSA in PBS } \\
\text { solution }\end{array}$ & $\begin{array}{l}\text { Polystyrene beads, } \\
\text { cells }\end{array}$ & $\begin{array}{l}\text { Incubate at } 20-25^{\circ} \mathrm{C} \text { in } \\
1 \text { hour }\end{array}$ & $\begin{array}{l}\text { - Used in CTC isolation test } \\
\text { - Minimize the adhesion } \\
\text { between beads/cells and the } \\
\text { channel walls }\end{array}$ \\
\hline $\begin{array}{l}\text { Fibronectin solution, } \\
100 \mu \mathrm{g} \mathrm{ml}^{-1}\end{array}$ & $\begin{array}{l}\text { Epithelial cells, cancer } \\
\text { cells }\end{array}$ & $\begin{array}{l}\text { Incubate at } 37^{\circ} \mathrm{C} \text { in more } \\
\text { than } 4 \text { hours }\end{array}$ & $\begin{array}{l}\text { - Used in interstitial flow } \\
\text { characterization experiments } \\
\text { - Create the adhesion } \\
\text { between cells and channel } \\
\text { walls }\end{array}$ \\
\hline $\begin{array}{l}\text { Poly-D-Lysine } \\
\text { (PDL) solution, } \\
1 \mathrm{mg} \mathrm{ml}^{-1}\end{array}$ & Collagen gel type I & $\begin{array}{l}\text { Incubate at } 37^{\circ} \mathrm{C} \text { in more } \\
\text { than } 4 \text { hours }\end{array}$ & $\begin{array}{l}\text { - Create the strong adhesion } \\
\text { between collagen gel and } \\
\text { channel walls } \\
\text { - Used in calibration test } \\
\text { where collagen gel plays as } \\
\text { an inert porous medium }\end{array}$ \\
\hline
\end{tabular}

The surface coating is conducted following these steps:

1) Prepare the coating solution according to experiment purposes. Table 3-1 indicates the usage of surface coating solutions with different purposes in this project.

2) Inject $50-100 \mu 1$ of coating solution into the channels.

3) Incubate the microfluidic device for a suitable duration (Refer to Table 3-1).

4) Withdraw the coating solution from the channels after incubating.

5) Wash the channels with sterile distilled water or PBS solution. 


\subsection{Cell culture}

In this project, we used the human breast cancer cells as the tumor cell models for our experiments. As being one of the most common types of cancer worldwide [155], the biological basis of breast cancer cells has been studied massively and become wellunderstood [156-158]. This provides us with proper and standard procedures and materials to work with breast cancer cell lines. Additionally, the well-studied breast cancer cell lines help us compare and validate the behaviors of the cells under variety of conditions.

We used two breast cancer cell lines MDA-MB-231 and MCF-7 (Michigan Cancer Foundation-7) from ATCC (Manassas, VA) in our in vitro experiments. Table 3-2 summarizes the usage of these cell lines in the experiments.

Table 3-2 Summary of cell lines used in the whole project.

\begin{tabular}{|l|l|l|l|}
\hline \multicolumn{1}{|c|}{ Cell lines } & \multicolumn{1}{|c|}{ Cell types } & \multicolumn{1}{c|}{ Culture medium } & \multicolumn{1}{c|}{ Experiment usage } \\
\hline MDA-MB-231, & Epithelial cells & $89 \%$ DMEM-12, 10\% & Used in CTC isolation tests. \\
breast cancer cells & & FBS, and 1\% Penicillin. & \\
breast cancer cell & Epithelial cells & $89 \%$ DMEM, 10\% FBS, & Used to form the cellular \\
& & and 1\% Penicillin- & aggregates in interstitial flow \\
& & Streptomycin & experiment. \\
\hline
\end{tabular}

\section{MDA-MB-231 Breast cancer cells}

The MDA-MB-231 human breast cancer cell line (ATCC, Manassas, VA) was cultured in the DMEM-12 medium containing 10\% Fetal Bovine Serum (FBS) and 1\% Penicillin (Thermo Fisher Scientific, Singapore). The cells were maintained at $37{ }^{\circ} \mathrm{C}$ with $5 \% \mathrm{CO}_{2}$ supply, with refreshment of the culture media every day. Once a 90\% confluent cell 
population is obtained, cell passage was performed with $0.25 \%$ trypsin-EDTA (Thermo Fisher Scientific, Singapore).

\section{MCF-7 Breast cancer cells}

The culture medium consisted of a mix of $89 \%$ Dulbecco's Modified Eagle Medium (DMEM), 10\% Fetal Bovine Serum (FBS), and 1\% Penicillin-Streptomycin. The cells were cultured in a $75 \mathrm{~cm}^{2}$ culture flask placed inside a $5 \% \mathrm{CO}_{2}$ incubator. When reaching confluency, the cells were carried on to aggregation production.

\section{Cell culture procedure}

1) The $75-\mathrm{cm}^{2}$ flask is used for this protocol; proportionally reduce or increase amount of dissociation medium for culture vessels of other sizes.

2) Remove the culture medium inside the flask.

3) Wash the attached cells with $10 \mathrm{ml}$ Phosphate Buffer Saline (PBS) for 3 times.

4) Add $3 \mathrm{ml}$ of $0.25 \%(\mathrm{w} / \mathrm{v})$ Trypsin $-0.53 \mathrm{mM}$ EDTA solution into the flask. Then put the flask in an incubator at $37^{\circ} \mathrm{C}$ and wait for the cells to detach from the flask surface in 3 to 5 minutes.

5) Observe the cells under an inverted microscope to determine when the cell layer is separated. Do not shake the flask to force the cells to be removed from the surface.

6) After that, add 6 to $8 \mathrm{ml}$ of the culture medium into the flask to deactivate trypsin, and aspirate the cells blended in the medium to a centrifuge tube.

7) Put the centrifuge tube including the suspension of cells and medium in a centrifuge machine, and spin at $1500 \mathrm{rpm}$ in 7 minutes.

8) Remove the supernatant.

9) Re-suspend the cells in $2 \mathrm{ml}$ of the fresh culture medium. 
10) Do the cells counting using hemocytometer: (1) Aspirate $10 \mu \mathrm{l}$ of cells suspension in trypan blue solution with the final concentration of trypan blue at $90 \%$.

(2) Transfer it into the hemocytometer and count at 4 corners.

11) Add appropriate aliquots of cell suspension to a new culture flask.

12) Put the flask into the incubator at $37{ }^{\circ} \mathrm{C}$ and $5 \% \mathrm{CO}_{2}$.

\subsection{Cellular aggregate production}

There are several methods to produce cellular aggregates [125]. Here we used the method proposed by Ryan et al. [159] as implemented by Douezan et al. [136].
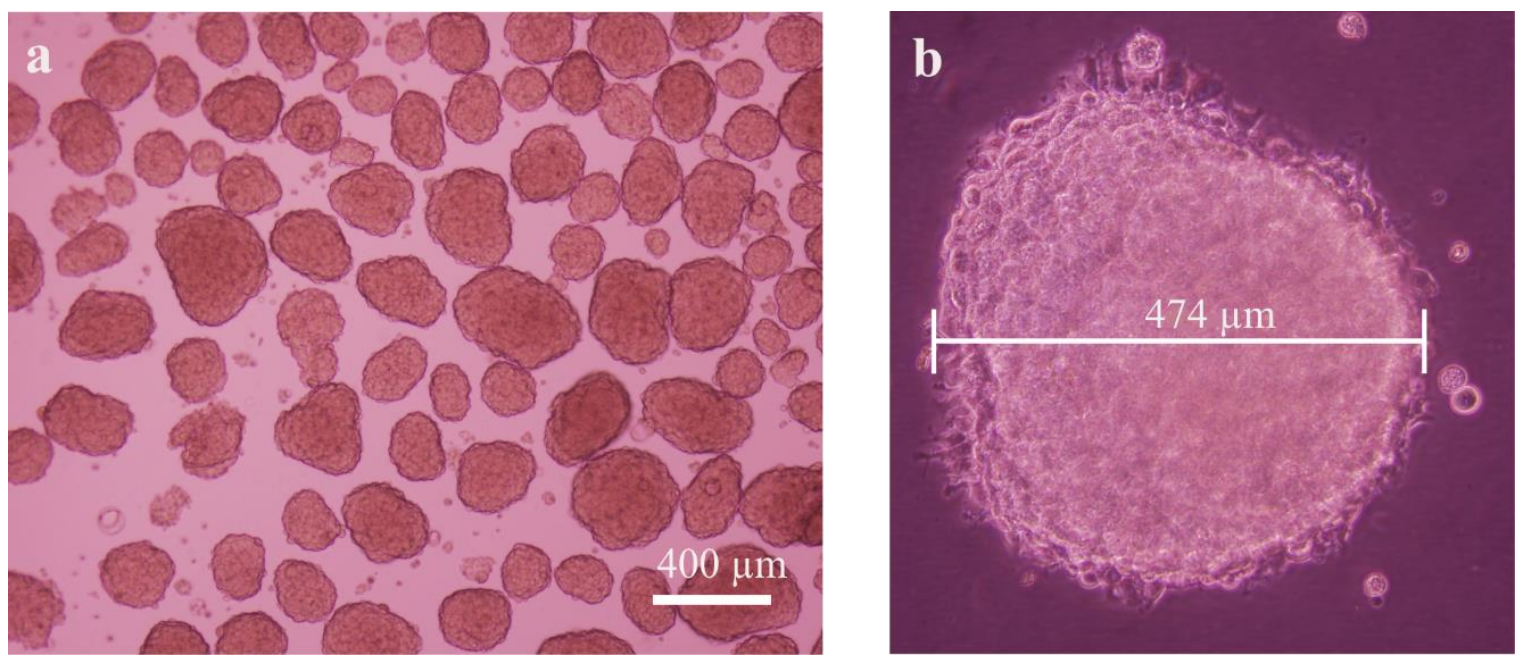

Figure 3.6 Cellular aggregates formed by orbital shaking. (a) Spheroidal cellular aggregates with dimensions ranging from $50-450 \mu \mathrm{m}$ in diameter. (b) Isolated view of a single cellular aggregate that was selected for experiments.

The detail procedure is described as following:

1) When reaching confluent, cells in the culture flask are treated with $0.25 \%(\mathrm{w} / \mathrm{v})$ Trypsin - $0.53 \mathrm{mM}$ EDTA and washed with culture medium to deactivate Trypsin. 
2) The cells are then re-suspended in $2 \mathrm{ml}$ fresh culture medium and transferred to a 25-ml Erlenmeyer flask containing $5 \mathrm{ml} \mathrm{CO}_{2}$-equilibrated culture medium at a concentration of $2 \times 10^{5}$ cell ml$^{-1}$.

3) Place the Erlenmeyer flask inside an incubated orbital shaker at $37^{\circ} \mathrm{C}$ spinning at 70 rpm for 24 hours.

4) The cell aggregates were achieved in spheroidal shapes with diameter ranging from 50 to $450 \mu \mathrm{m}$ (Figure 3.6). The aggregates were selected and transferred to a 35-mm petri dish to be ready for experiments. 


\section{CHAPTER 4}

\section{Sequential isolation of floating cancer cells under continuous flow}

This chapter introduces our development of a microfluidic device to isolate floating cancer cells automatically and sequentially under continuous flow.

We carry out a simulation study to investigate the preliminary parameters for the microfluidic design. We begin our study by validating our isolating theory with $80 \mu \mathrm{m}$ microbeads isolation in an 'enlarged device', before coming up with the 'true scale' device for cancer cell isolation.

In the last part of this chapter, the results of the device successfully isolating either the proof-of-concept microbeads and the real cancer cells are shown with $100 \%$ trapping yield and > $95 \%$ efficiency. This device also demonstrates its sorting ability of particles in different sizes, which can be feasible in circulating tumor cell detection and particle separation. 


\subsection{Simulation study}

We performed numerical simulations to optimize the sieve separation distance by calculating the flow field around a microsieve trapped with a particle, located in the middle of a straight channel. The simulation was applied with the assumptions that the flow is a fully developed Stokes' flow, the fluid is a Newtonian fluid. In the absence of the microsieve, the flow everywhere in the channel is unidirectional. The presence of a microsieve forces the upstream unidirectional streamlines to bend around the sieve and the flow becomes unidirectional again further downstream. The unidirectionality of the flow at any arbitrary location $(x, y, z)$ can be quantified by introducing a dimensionless unidirectional factor,

$$
\varepsilon_{v}(x, y, z)=\sqrt{\left(v^{2}+w^{2}\right) /\left(u^{2}+v^{2}+w^{2}\right)},
$$

where the velocity components $u, v, w$ are in the $x, y$ and $z$ directions, respectively. When the flow field is only in the $x$-direction, $v$ and $w$ are zeros, and thus the dimensionless unidirectional factor, $\varepsilon_{v}=0$. On the other hand, a non-zero $\varepsilon_{v}$ corresponds to nonunidirectional flow field. We define $x_{\text {sieve }}$ as the reference $x$ position measured from the center of the microsieve (Figure 4.1a). We calculated the average $\varepsilon_{V}$ over the cross section area,

$$
\bar{\varepsilon}_{v}\left(x_{\text {sieve }}\right)=\iint \varepsilon_{v}\left(x_{\text {sieve }}, y, z\right) d y d z
$$

The result indicates that a separation of $>500 \mu \mathrm{m}$ can ensure the flow to recover unidirectionally where $\bar{\varepsilon}_{V}\left(x_{\text {sieve }}\right)<0.005$ (Figure $4.1 \mathrm{~b}$ ). Therefore, the second microsieve has to be located at least $500 \mu \mathrm{m}$ downstream from the first one. 
a

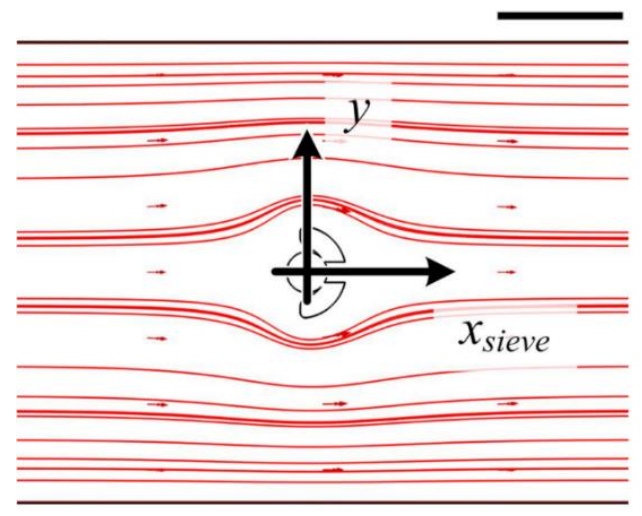

b

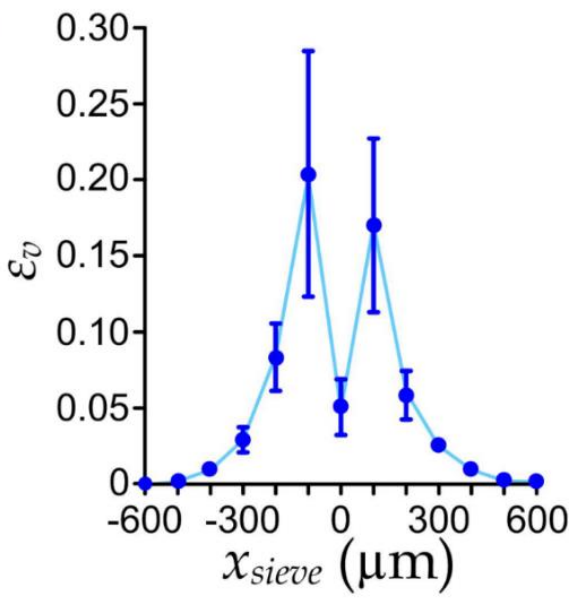

Figure 4.1 (a) Velocity profile at the mid-height plane around a microsieve structure at the flow rate of 100 $\mu 1 \mathrm{~min}^{-1}$. (b) dimensionless unidirectional factor $\varepsilon_{v}$ as a function of $x_{\text {sieve }}$. Scale bar: $200 \mu \mathrm{m}$. Error bars are standard deviations. Adapted from Tran et al. [56] with permission of the Royal Society of Chemistry.

\section{Flow profile simulation}

We applied simulation software COMSOL Multiphysics (COMSOL, Burlington, MA) to quantify the stabilized streamlines and local velocities of a microchannel section containing a microsieve structure. The total flow rate was set as $100 \mu 1 \mathrm{~min}^{-1}$. We adopted the dimensions of the 'enlarged device' for the numerical simulation.

\subsection{Experimental setup}

\section{Validation test with microbeads using 'enlarged device'}

For the microbead isolation experiments with the 'enlarged device', we prepared the microbead sample by diluting a stock solution of $80 \mu \mathrm{m}$ diameter polystyrene beads (Duke 4K-80, size distribution of $79.4 \pm 0.5 \mu \mathrm{m}$, ThermoFisher Scientific, Pittsburgh, PA, USA) down to a bead density of $3 \times 10^{3}$ bead $\mathrm{ml}^{-1}$. Polystyrene microbeads were used in this study as they are produced in spherical shape with a wide range of sizes in high precision but at low cost. The density of polystyrene beads is $1.05 \mathrm{~g} \mathrm{ml}^{-1}$, which makes the beads stay floating when being moved by the flow and have less contact with the 
substrate of the device. Moreover, since polystyrene beads are standardized and resistant to acids, bases and alcohol, they are commonly used in various applications and experiments related to particle counting and tracing.

We injected water as buffer through the side-channel inlet at $10 \mu 1 \mathrm{~min}^{-1}$, while clamping the tubing of the center inlet and the outlet ports for approximately 3 minutes, to remove the trapped air bubbles. Once all bubbles disappeared, we removed the clamps and injected the sample to the center inlet of the trapper device at a flow rate of $2 \mu 1 \mathrm{~min}^{-1}$ with a syringe pump (NE-1002X, New Era Pump Systems, Farmingdale, NY). At the same time, we injected water as buffer to the side inlet of the device at a flow rate of $40 \mu 1 \mathrm{~min}^{-1}$. The sample flow was sandwiched by the side buffers, and focused along the axial center of the microchannel. The trapping process was recorded using a high speed camera (Axiocam 506 Color, Zeiss, Jena, Germany) installed to an inverted microscope (Axio Observer.Z1, Zeiss). The experiments ended when all microsieves were filled with the polystyrene beads which occurred in approximately 5-10 minutes.

\section{Isolation test with microbeads and cancer cells using 'true-scale' device}

Similarly, for the validation of the 'true-scale' sequential microtrapper, we conducted the isolation experiment with $20 \mu \mathrm{m}$ diameter fluorescent polystyrene microbeads (cat\# FP-20052-5, size distribution of $18-24.9 \mu \mathrm{m}$, Spherotech, Lake Forest, IL, USA) and MDA-MB-231 human breast cancer cell. The experimental procedures were the same as previously described for the 'enlarged device' experiments, with flow rates of $2 \mu 1 \mathrm{~min}^{-1}$ and $40 \mu 1 \mathrm{~min}^{-1}$ for the bead sample and side liquid (water), respectively. For the breast cancer cell isolation experiments, the sample solution was prepared by harvesting MDA cells using $0.25 \%$ trypsin-EDTA (Thermo Fisher Scientific) and diluted the cells to a density of $5 \times 10^{4}$ cell $\mathrm{ml}^{-1}$ in culture media. 


\section{Live/dead cell test}

After the sequential cell isolation experiment at a continuous flow rate of $3 \mu 1 \mathrm{~min}^{-1}$ for 30 min, the sample solution was replaced by reagents of the LIVE/DEAD Cell Viability kit (cat\# L-3224, Thermo Fisher Scientific) with a continuous flow from both the sample inlet (flow rate: $0.1 \mu 1 \mathrm{~min}^{-1}$ ) and buffer inlet (flow rate: $2 \mu 1 \mathrm{~min}^{-1}$ ) to stain different fluorescence signals for live and dead cells. The flow of staining solution was maintained for $20 \mathrm{~min}$, followed by replacing the solution with water for capturing fluorescence images (Axiocam 506 Color, Zeiss, Jena, Germany) under an inverted fluorescence microscope (Axio Observer.Z1, Zeiss).

\subsection{Results and discussions}

\subsubsection{Performance parameter of isolation device}

For optimization of the designed microsieves, trapping rate is an important readout since it determines the performance of the device. The trapping rate is defined as the percentage of cells/beads flowing into the device isolated in the correct sequence. All the devices have 50 microsieves along the main channel, and the number of cells/beads trapped and escaped throughout the 50 microsieves will determine the trapping rate of each device, as followed by this equation:

$$
\text { Trapping rate, } \eta=\frac{\text { Particles }_{\text {trapped in sequence }}}{\text { Particles }_{\text {trapped }}+\text { Particles }_{\text {escaped }}} \times 100 \% \text {. }
$$

As the bead and cell concentration had been diluted in the preparation step, the cells/beads flowing into the main channel were trapped in sequence. The trapping rate counts until all the microsieves are filled. As the trapping rate only counts the cells/beads 
isolated in sequence, we also define isolation yield as a parameter to estimate the trapping performance of the devices without the need of sequential isolation.

$$
\text { Isolation yield }=\frac{\text { Particles }_{\text {trapped }}}{\text { Particles }_{\text {trapped }}+\text { Particles }_{\text {escaped }}} .
$$

\subsubsection{Concept validation using microbeads}

We validated with experiments, for the isolation of $80 \mu \mathrm{m}$ beads, that an initial offset of $15 \mu \mathrm{m}$ in the positive $y$-direction could achieve isolation of the first microbead, while deflecting the flow path of the subsequent incoming beads to the intended trajectory through the first sieve in the negative $y$-direction (data not shown). As a proof-of-concept, we first configured the sieve offset to be $45 \mu \mathrm{m}$ and performed the bead isolation experiment. The key snapshots of the sequential isolation are shown in Figure 4.2a. This result demonstrates the feasibility of our sequential isolation strategy.

We performed a parametric study to characterize the trapping rate as a function of the 'sieve offset' ranging from $35 \mu \mathrm{m}$ to $50 \mu \mathrm{m}$ (Figure $4.2 \mathrm{~b}$ ). Our results show that the 'sieve offset' of $40 \mu \mathrm{m}$ can achieve the highest trapping rate of $\sim 98 \%$ for microbeads with a diameter of $80 \mu \mathrm{m}$. 
a
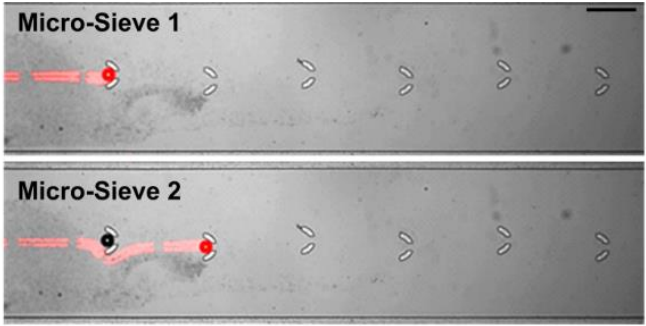

Micro-Sieve 6 b

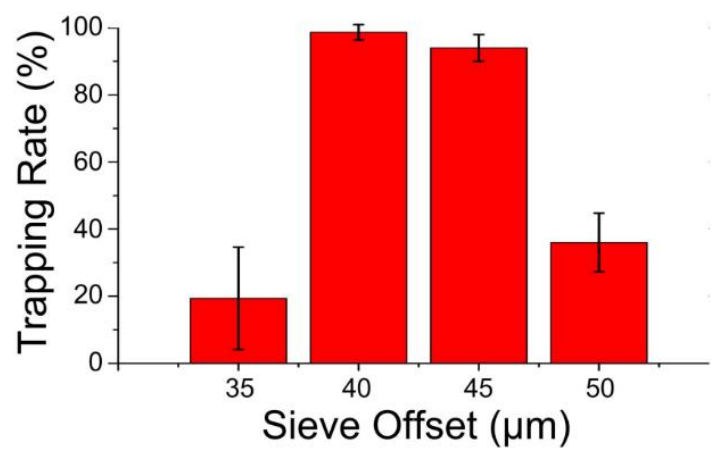

Figure 4.2 (a) Sequential isolation process of microbeads. The first bead enters was trapped in microsieve 1; and subsequently the second bead bypassed the first filled trapper and was trapped in microsieve 2 . This process continued until all the trappers are filled in a sequential manner. Trace of the bead movements was highlighted in red. Scale bar: $200 \mu \mathrm{m}$. (b) Trapping rate as a function of sieve offset for the 'enlarged device'. Error bars are standard deviations. $N \geq 5$. Adapted from Tran et al. [56] with permission of the Royal Society of Chemistry.

Having demonstrated the sequential isolation concept using the larger microbeads of $80 \mu \mathrm{m}$, we proceeded to fabricate and test the sequential isolation of $20 \mu \mathrm{m}$-diameter microbeads using the 'true-scale' microdevice by scaling down the model device with a 4:1 geometric ratio (i.e. $225 \mu \mathrm{m}$ channel width, $30 \mu \mathrm{m}$ channel height, inner sieve diameter: $25 \mu \mathrm{m}$, outer sieve diameter: $35 \mu \mathrm{m}$; sieve gap width: $10 \mu \mathrm{m}$, initial offset: $3.75 \mu \mathrm{m}$, sieve offset: $10 \mu \mathrm{m}$ and separation distance: $150 \mu \mathrm{m})$. The corresponding scaled Reynolds number was very small and therefore the flow streamlines were identical to the enlarged device. To verify the sequential isolation capability, microbeads with the diameter of $20 \mu \mathrm{m}$ (density: $5 \times 10^{3}$ bead $\mathrm{ml}^{-1}$ ) were injected into the true-scale device with the sample flow rate of $2 \mu 1 \mathrm{~min}^{-1}$ and buffer flow rate of $40 \mu 1 \mathrm{~min}^{-1}$. The results (Figure 4.3) indicate an effective sequential isolation of this device with a similar trapping rate $(\geq 96 \%)$. 

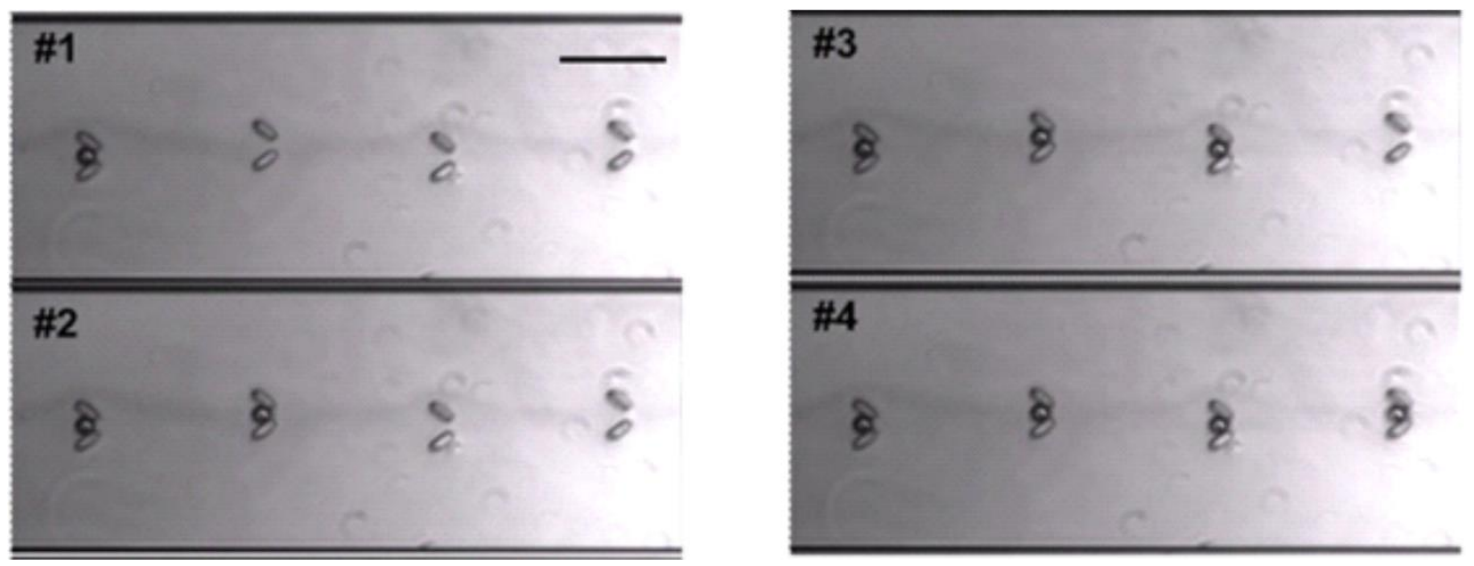

Figure 4.3 Sequential isolation of microbeads with a $20 \mu \mathrm{m}$ diameter in micro-sieves (top to bottom) using a 'true-scale' microdevice. Scale bar: $100 \mu \mathrm{m}$. Adapted from Tran et al. [56] with permission of the Royal Society of Chemistry.

\subsubsection{Isolation of size variant microbeads}

We further applied the device to examine the feasibility of the sequential isolation of a mixture containing microbeads of different sizes. We prepared two mixture samples by mixing $80 \mu \mathrm{m}$ microbeads $\left(3 \times 10^{3}\right.$ bead $\left.\mathrm{ml}^{-1}\right)$ with $20 \mu \mathrm{m}$ microbeads $\left(3 \times 10^{4}\right.$ bead ml$\left.^{-1}\right)$ and $8 \mu \mathrm{m}$ microbeads $\left(10^{6}\right.$ bead $\left.\mathrm{ml}^{-1}\right)$, respectively; and the representative micrographs of the cell isolation are shown in Figure 4.4. For this test, the $80 \mu \mathrm{m}$ and $20 \mu \mathrm{m}$ microbeads are used from the same types as the previous tests, the $8 \mu \mathrm{m}$ microbeads is from Duke $4 \mathrm{k}$, cat\# 4208A, ThermoFisher Scientific, Pittsburgh, PA, USA, with size distribution of $7.979 \pm 0.055 \mu \mathrm{m}$. With the presence of the $40 \mu \mathrm{m}$ gap at the center of each microsieve, the beads which are smaller than the gap width will flow through the microsieve without being trapped. When the $8 \mu \mathrm{m}$ and $20 \mu \mathrm{m}$ microbeads move to the empty (or unfilled) microsieves, they will pass through the gaps of the empty microsieves. When moving to a microsieve which is currently filled with an $80 \mu \mathrm{m}$ bead, the $8 \mu \mathrm{m}$ and $20 \mu \mathrm{m}$ microbeads will bypass that microsieve. Our experimental results indicate that all the $80 \mu \mathrm{m}$ beads in the mixture samples were captured in the microsieves while all the smaller microbeads flow to the outlet without being trapped by the microsieve structures. Therefore, the presence of the smaller microbeads would not induce significant flow variations large 
enough to affect the trajectories of the $80 \mu \mathrm{m}$ beads, and thus not impacting the isolation performance of the larger particles.

Notably, the diameter of CTCs is typically $15-25 \mu \mathrm{m}[160,161]$, red blood cells has an effective diameter of $5-6 \mu \mathrm{m}$ (as the major diameter is $6-8 \mu \mathrm{m}$ and the thickness is $\sim 3 \mu \mathrm{m}$ ) [162] and the diameter of platelets is $2-3 \mu \mathrm{m}$ [163]. On the other hand, approximately $95 \%$ of leukocytes have dimensions less than $15 \mu \mathrm{m}$ (neutrophil $\sim 62 \%$, $10-14 \mu \mathrm{m}$; eosinophil $\sim 2.3 \%, 10-14 \mu \mathrm{m}$; basophil $\sim 0.4 \%, 10-14 \mu \mathrm{m}$; lymphocyte $\sim 30 \%, 8-10 \mu \mathrm{m}$; and monocyte $\sim 5.3 \%, 15-20 \mu \mathrm{m}$ ) [164]. Thus, the proposed sequential isolation scheme is applicable to extract CTCs from a blood sample containing abundant red blood cells, platelets, and majority of the white blood cells. The small amount of white blood cells trapped can be further differentiated from the CTCs through on-chip fluorescence immuno- staining [71], fluorescence- activated cell sorting (FACS) [165], or immuno- magnetic separation [165], or density gradient centrifugation [166].

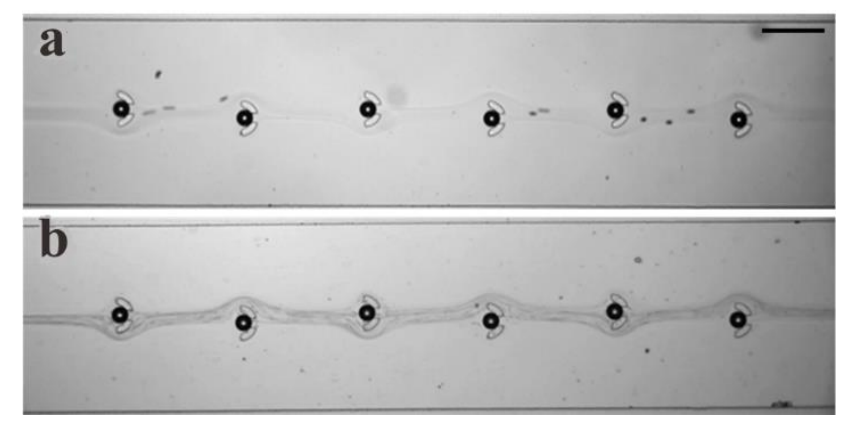

Figure 4.4 Isolation of $80 \mu \mathrm{m}$ microbeads from (a) the mixture of $80 \mu \mathrm{m}$ beads and $20 \mu \mathrm{m}$ beads, and (b) the mixture of $80 \mu \mathrm{m}$ beads and $8 \mu \mathrm{m}$ beads, implemented on the enlarged device. Scale bar: $200 \mu \mathrm{m}$. Adapted from Tran et al. [56] with permission of the Royal Society of Chemistry.

\subsubsection{Sequential cancer cell isolation}

Unlike rigid microbeads, live-cells are deformable in the microsieves under hydrodynamic pressure; and such deformation is time-dependent due to the cell viscoelasticity $[167,168]$. Therefore, it is necessary to investigate the allowable flow rate 
and duration for the successful live-cell encapsulation. We performed the sequential isolation experiments of human breast cancer cells (MDA-MB-231) using the 'true-scale' microdevice under different sample flow rates $\left(3 \mu 1 \mathrm{~min}^{-1}, 6 \mu 1 \mathrm{~min}^{-1}\right.$ and $\left.9 \mu 1 \mathrm{~min}^{-1}\right)$. We used the same 'true-scale' device in the $20 \mu \mathrm{m}$ microbeads isolation test ( $225 \mu \mathrm{m}$ channel width, $30 \mu \mathrm{m}$ channel height, inner sieve diameter: $25 \mu \mathrm{m}$, outer sieve diameter: $35 \mu \mathrm{m}$; sieve gap width: $10 \mu \mathrm{m}$, initial offset: $3.75 \mu \mathrm{m}$, sieve offset: $10 \mu \mathrm{m}$ and separation distance: $150 \mu \mathrm{m})$. We configured the buffer flow rates to be 20 times the sample flows, adopting the same volumetric ratios as the 'enlarged' device. The average velocity $V_{\text {avg }}$ of the flow at the main channel in cases of applying sample flow rates of $3 \mu 1 \mathrm{~min}^{-1}$, $6 \mu 1 \mathrm{~min}^{-1}$ and $9 \mu 1 \mathrm{~min}^{-1}$ is calculated to be $155.5 \mathrm{~mm} \mathrm{~s}^{-1}, 311.1 \mathrm{~mm} \mathrm{~s}^{-1}$ and $466 \mathrm{~mm} \mathrm{~s}^{-1}$, respectively. Since the main microchannel has the aspect ratio between channel width and channel height at 7.5:1, we can consider the flow in this thin channel as plane Poiseuille flow. Thus, the average shear rate $\overline{\dot{\gamma}}$ of the flow induced in the channel is calculated as:

$$
\overline{\dot{\gamma}}=\frac{3 V_{\text {avg }}}{2 h}
$$

where $h$ is the channel height. With the sample flow rates of $3 \mu 1 \mathrm{~min}^{-1}, 6 \mu 1 \mathrm{~min}^{-1}$ and $9 \mu 1 \mathrm{~min}^{-1}$, the average shear rate in the channel is $7775 \mathrm{~s}^{-1}, 15555 \mathrm{~s}^{-1}$ and $23300 \mathrm{~s}^{-1}$, respectively.

Here, we consider the encapsulated cell remaining in the microsieve for more than $30 \mathrm{~min}$ as a successful isolation. Our results (Figure 4.5a) show that the sample flow rate of $3 \mu 1 \mathrm{~min}^{-1}$ with the average shear rate of $7775 \mathrm{~s}^{-1}$ achieved the required isolation, whereas the flow rate of $9 \mu 1 \mathrm{~min}^{-1}$ with the average shear rate of $23300 \mathrm{~s}^{-1}$ was clearly beyond the workable pressure range. The flow rate of $6 \mu 1 \mathrm{~min}^{-1}$ with the average shear rate of $15555 \mathrm{~s}^{-1}$ induced an unfavorable cell condition after $30 \mathrm{~min}$, where the cell body 
stayed partially outside the microsieve. This indicates that the microsieve gap was likely to squeeze the inner cellular structures including the nucleus.

a
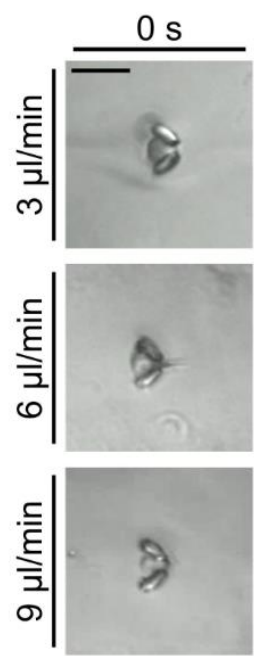
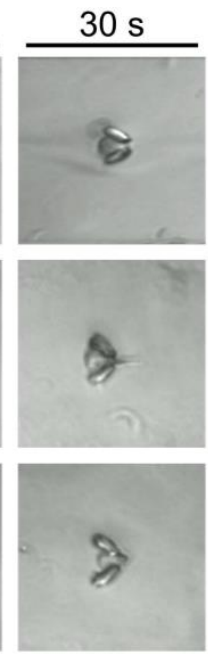
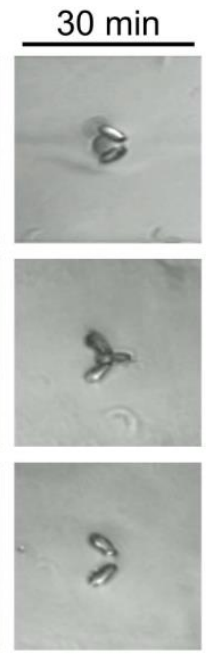

b

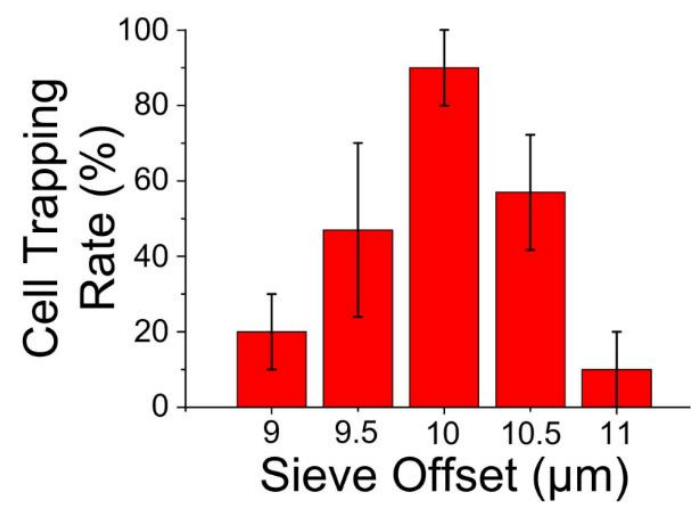

Figure 4.5 (a) Microscopic snapshots of cells trapped in 'true-scale' microsieves for different durations of encapsulation ( $0 \mathrm{~s}, 30 \mathrm{~s}$ and $30 \mathrm{~min})$ under different sample flow rates $\left(3 \mu 1 \mathrm{~min}^{-1}, 6 \mu 1 \mathrm{~min}^{-1}\right.$ and $\left.9 \mu 1 \mathrm{~min}^{-1}\right)$ corresponding with different average shear rates $\left(7775 \mathrm{~s}^{-1}, 15555 \mathrm{~s}^{-1}\right.$ and $\left.23300 \mathrm{~s}^{-1}\right)$. Scale bar: $50 \mu \mathrm{m}$. (b) Trapping rate of cancer cells MDA-MB-231 as a function of the sieve offset, ranging $9-11 \mu \mathrm{m}$. Error bars are standard deviations. $N \geq 5$. Adapted from Tran et al. [56] with permission of the Royal Society of Chemistry.

We characterize the cell trapping rate of MDA-MB-231 for different sieve offsets, ranging from $9 \mu \mathrm{m}$ to $11 \mu \mathrm{m}$ (with steps of $0.5 \mu \mathrm{m}$ ) under the sample flow rate of $3 \mu 1 \mathrm{~min}^{-1}$ and the buffer flow rate of $60 \mu 1 \mathrm{~min}^{-1}$, with the procedures described in 4.2 . The isolation performance of the 'true-scale' device (Figure $4.5 \mathrm{~b}$ ) perfectly agrees with the 'enlarged device' (Figure 4.2b). The 'enlarged device' has an optimal sieve offset of $40 \mu \mathrm{m}$ with trapping rate of $\sim 98 \%$; while the scaled down 'true scale' device with $4: 1$ geometric ratio, has an optimal sieve offset of $10 \mu \mathrm{m}$ with the highest cell trapping rate of $\sim 90 \%$. Occasionally, we observed a few cells were not trapped in the device in sequence. This could be due to a suboptimal sieve offset, and other factors including the Brownian motion effect of microparticles and the pressure fluctuations generated in the syringe pump. The trapping rate represents the percentage of cell isolation in the correct 
sequence. The escaped cells are recaptured in the following microsieves downstream in the device. The total number of microsieves for the 'true-scale' device is 50 .

Furthermore, we examined the effect of continuous flow in the microfluidic isolation on the cell viability by examining the ratios of live/dead cells before injection and after isolation (under a continuous flow of $\geq 30 \mathrm{~min}$ ) in the microdevice (both included $\sim 100 \%$ of live cells in populations). Figure 4.6 shows a representative brightfield and fluorescent micrographs of MDA cells after treatment of the live/dead cell viability assay. We observed that the sequential cell isolation does not affect the cell viability. It has been reported by Barnes et al. that $\sim 100 \%$ viability of cancer cells can be maintained for shear stress $<100$ dyne $\mathrm{cm}^{-2}[169,170]$, which agrees perfectly with the cell viability result in the sequential isolation microdevice with induced shear stresses on trapped cells in the scale of $\sim 3.7 \mathrm{dyne}^{-2}$ (considering the average flow velocity was $\sim 11.1 \mathrm{~mm} \mathrm{~s}^{-1}$ and the characteristic length was the channel height $\left.30 \mu \mathrm{m}\right)$. More importantly, the microdevice can provide a unique advantage of knowing the predefined cell positions after isolation, implying that efforts for scanning and cells monitoring operations can be largely reduced. For instance, microscopic images of the trapped cells can be implemented by scanning along the microsieve positions in sequence. The cell viability test has also demonstrated the compatibility of this microdevice in general cell analysis applications, including drug treatments and specific staining techniques [171].

Alternative continuous-flow particle focusing techniques based on acoustic [172] or physical [173] effects can be further integrated with the sequential isolation scheme reported here such that no side buffer flow is required and the throughput of each of the microdevices can be increased to $\sim 63 \mu 1 \mathrm{~min}^{-1}$. The cell isolation throughput can be further enhanced by pumping the sample into the multiple (around $5-10$ ) microdevices 
working in parallel, in order to reach the required standard blood processing volume

$(\geq 7.5 \mathrm{ml})$ within a manageable timeframe of $\sim 1$ hour [174].

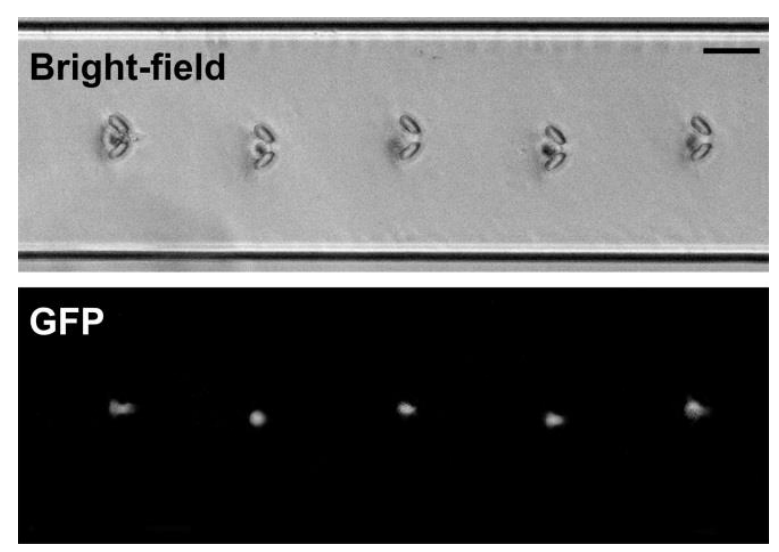

Figure 4.6 Representative bright-field (upper) and fluorescence (lower) microscopic images of MDA cells after treatment of the live/dead cell viability assay. Presence of fluorescence signals for all the trapped cells indicates their viability. Scale bar: $50 \mu \mathrm{m}$. Reproduced from Tran et al. [56] with permission of the Royal Society of Chemistry. 


\section{CHAPTER 5}

\section{Microfluidic study of interstitial flow through a model tumor}

This chapter presents our research on interstitial flow and its biophysical effects on the dynamics of a model tumor.

The chapter starts by developing a theoretical model to characterize a flow moving through a simple poroelastic material model.

In the next part, we introduced two experiments with two designs of microfluidics to characterize interstitial flow in two conditions: constant flow rate and continuously increasing flow rate.

Our findings on the effects of interstitial flow on the behavior of a model tumor are discussed in the last part of this chapter. Our experimental results demonstrate the existence of two mechanical regimes in the behavior of a tumor mass under flow. The transition between these two regimes corresponds to flow-induced fracture occurring inside the tumor. These findings will serve as a basis to model the mechanics of flow through a tumor and to understand how interstitial flow contributes to the detachment of cells from the tumor mass, a key step in cancer metastasis. 


\subsection{Theoretical poroelastic model for flow through a cellular aggregate}

We started the study by developing a theoretical model for a cell aggregate considering the aggregate to behave as a poroelastic material under interstitial flow.

First, we consider a model including a poroelastic cylindrical tube with radius $R$ and length $L$. This poroelastic tube is axially covered by a rigid outer shell. A fluid with dynamic viscosity $\mu$ flowing through the tube with an average velocity $v$, resulted from a pressure difference $\left(P-P_{0}\right)$ at two ends of the tube (with $P_{0}$ is the background pressure).
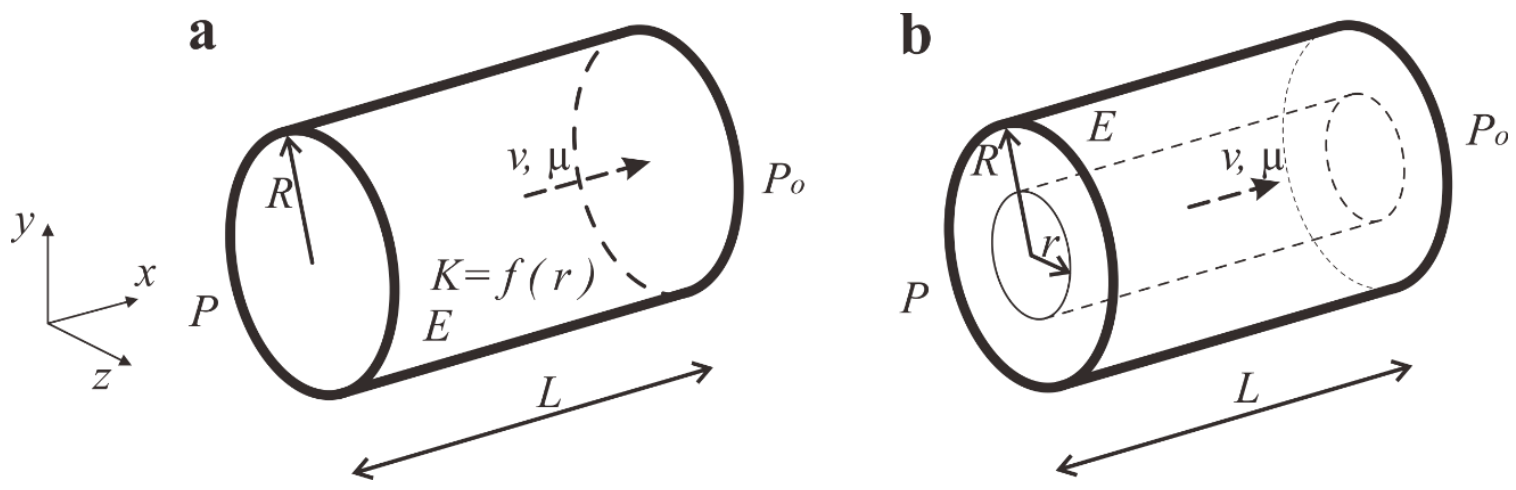

Figure 5.1 Our theoretical model using poroelasticity to explain the behavior of the cellular aggregate under the flow. (a) The model in the point of view of a porous media flow. It includes a homogeneous porous cylindrical tube with radius $R$ and permeability $K$ where $K$ is a function of $r$, as described by Equation (3.6). (b) The model in the point of view of elasticity. It is an elastic cylindrical tube of radius $R$ with an inner empty hole of radius $r$ and an outer layer of elasticity $E$. This hole of radius $r$ is a simplified representation of the total pores of the model. A pressure difference $\left(P-P_{0}\right)$ is applied through it. Assume that the deformation in all direction is equal.

From the point of view of a porous media flow, the tube is considered as a homogeneous porous cylinder of radius $R$ with an effective permeability $K$.

From the point of view of elasticity, we assume the model is a cylinder with an inner empty hole of radius $r$ and an outer layer of elasticity with Young Modulus $E$. This hole of radius $r$ is a simplified representation of all the pores in the porous material. Its cross-sectional area is equal to the sum of all cross-sectional areas of pores in the real material. Thus, the porosity of the real material is defined as an equivalent global 
porosity, corresponding to the permeability given by the ratio between the hole size and the total cylinder size.

Assume that the deformation in all directions is equal. Starting from Darcy's law for one-dimensional flow through a porous medium with constant cross-section area, the average velocity of fluid going through the tube follows equation (2.3):

$$
v=-\frac{K}{\mu} \frac{\partial P}{\partial x}
$$

Assume that

$$
-\frac{\partial P}{\partial x}=\frac{\left(P-P_{0}\right)}{L}=\frac{\Delta P}{L}
$$

and

$$
v=\frac{Q}{\pi R^{2}}
$$

where $Q$ is the flow rate across the poroelastic tube.

The permeability of the porous material can be further simplified from the standard Kozeny-Carman equation [175-177], resulting in:

$$
K=\alpha \frac{r^{6}}{R^{6}}
$$

The Kozeny-Carman equation assumes flow to occur through a parallel ensemble of tortuous tubes of similar length, the dimensions of which correspond to the pores in a packed bed of spherical particles. Laminar flow in the tubes is assumed. Moreover, the version of the equation used in the text is simplified by assuming porosity $\phi$ to be much smaller than 1 (low porosity limit). The Kozeny-Carman equation is a simple model to describe permeability, and we use as a first attempt to characterize our system. 
Equation (2.3) then becomes:

$$
\frac{Q}{\pi R^{2}}=\frac{\alpha}{\mu L} \frac{r^{6}}{R^{6}} \Delta P
$$

We define $r_{i}$ as the initial radius of the inner empty hole of the model in elasticity perspective, before any deformation:

$$
r=r_{i} \text { when } P=P_{0}=0 \text {. }
$$

The pressure difference causes a linear elastic deformation to the tube, which is defined as:

$$
\varepsilon=\frac{\sigma}{E}=\frac{P}{E}
$$

with

$$
\varepsilon=\frac{r-r_{i}}{R-r_{i}}
$$

where $\varepsilon$ is the relative displacement of the elastic region, $\sigma$ is the compressing stress of the pressure on the elastic region.

We then non-dimensionalize the variables as:

$$
\tilde{p}=\frac{P}{E}, \tilde{r}=\frac{r}{R},
$$

and achieve:

$$
\tilde{r}=\tilde{r}_{i}+\left(\tilde{p}_{0}+\Delta \tilde{p}\right)\left(1-\tilde{r}_{i}\right) \text {, note that } \tilde{r} \leq 1 ; \tilde{p} \leq 1 \text {. }
$$

Substitute (5.8) to (5.4), we have: 


$$
\frac{Q \mu L}{\alpha R^{2} E}=\pi \tilde{r}^{6} \Delta \tilde{p}
$$

We continue to non-dimensionalize

$$
\tilde{Q}=\frac{Q \mu L}{\alpha R^{2} E}
$$

and combine (5.9) with (5.10) to achieve:

$$
\tilde{Q}=\pi \tilde{r}^{6} \Delta \tilde{p}=\pi \Delta \tilde{p}\left[\tilde{r}_{i}+\left(\tilde{p}_{0}+\Delta \tilde{p}\right)\left(1-\tilde{r}_{i}\right)\right]^{6}
$$

We look at some limiting cases of the model:

i. When $\tilde{r}_{i} \rightarrow 0$, the empty hole is insignificant initially or the total volume of all the pores inside the material is close to zero. Then, equation (5.12) becomes:

$$
\tilde{Q}=\pi \Delta \tilde{p}\left(\tilde{p}_{0}+\Delta \tilde{p}\right)^{6}
$$

a) If $\tilde{p}_{0} \rightarrow 0$, the background pressure $P_{0}$ is atmospheric pressure and it does not create any deformation in the tube. As the pore fraction is insignificant at the beginning, the pressure difference will first result in an elastic deformation of the whole tube in all directions. This makes the pore fraction increase with the square of radius $\tilde{r}=\Delta \tilde{p}=\varepsilon$. The flow rate across the tube in this case is calculated as:

$$
\tilde{Q}=\pi \Delta \tilde{p}^{7}
$$


b) If $\tilde{p}_{0} \rightarrow 1$, The elastic region will be fully compressed. Our linear elastic model is no longer valid in this limit as we need to use non-linear elasticity for the large deformation.

The tube then becomes an empty tube (i.e., an infinitely porous tube) with a rigid shell at radius $\tilde{r}_{i}=1$. The flow going through the tube will behave as Poiseuille flow:

$$
\tilde{Q}=\pi \Delta \tilde{p}
$$

ii. When $\tilde{r}_{i} \rightarrow 1$, the whole tube is an empty region. This case is thus formally identical to case i.b:

$$
\tilde{Q}=\pi \Delta \tilde{p}, \text { with } r=r_{i}=R \text {. }
$$

iii. When $\tilde{r}_{i} \rightarrow 0.5$, the model tube now consists of a total pore area with radius $r_{i}=R / 2$ at the beginning. Equation (5.12) becomes:

$$
\tilde{Q}=\frac{\pi}{2^{6}} \Delta \tilde{p}\left(1+\tilde{p}_{0}+\Delta \tilde{p}\right)^{6}
$$

a) If $\tilde{p}_{0} \rightarrow 0, P_{0}$ is atmospheric pressure and the tube is not initially compressed. The pressure difference applied to the tube will expand the pore fraction to radius $\tilde{r}=0.5(1+\Delta \tilde{p})=0.5(1+\varepsilon)$. The flow rate going through the tube is:

$$
\tilde{Q}=\frac{\pi}{2^{6}} \Delta \tilde{p}(1+\Delta \tilde{p})^{6}
$$


b) If $\tilde{p}_{0} \rightarrow 1$, we again recover case i.b.

$$
\tilde{Q}=\pi \Delta \tilde{p}
$$

The results from the theoretical poroelastic model are plotted in Figure 5.2. Same as in the cell aggregate experiment, we plot the relation between flow rate and the pressure difference across the poroelastic medium (black curves). We also show how the radius of the pore region varies with applied pressure difference (red curves). Corresponding to the discussion above, we present results for two values of the background pressure $\left(\tilde{p}_{0}=0\right.$ and $\left.\tilde{p}_{0}=0.5\right)$ and for three different values of the initial equivalent pore radius $\left(r_{i}=0, r_{i}=R / 2\right.$ and $\left.r_{i}=R\right)$. First, we observe that the equivalent pore radius $r$ increases linearly with applied pressure, until it becomes equal to the radius of the tube.

$\mathbf{a}$

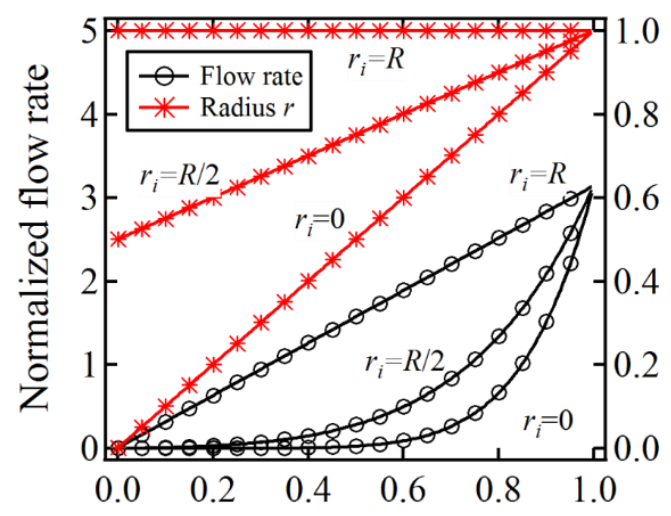

Normalized pressure difference b

$$
\tilde{p}_{0}=0.5
$$

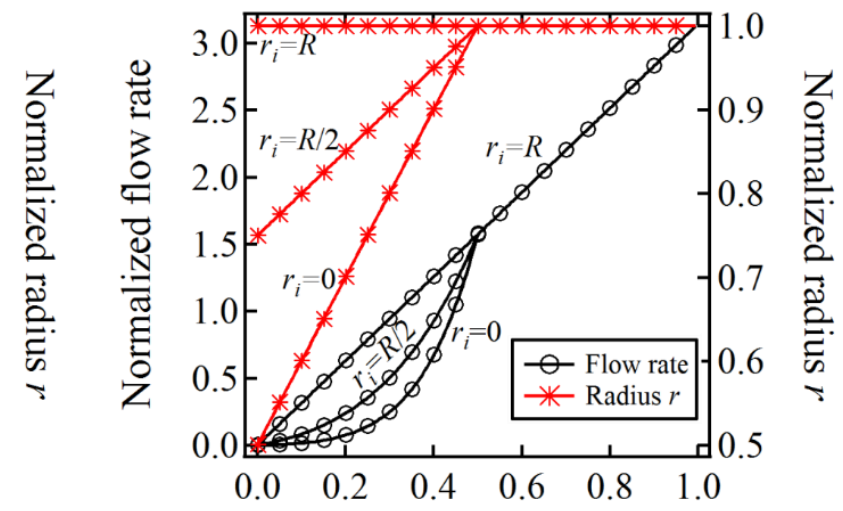

Normalized pressure difference

Figure 5.2 Results from theoretical model of interstitial flow through a cylindrical poroelastic tube including a porous core with radius $r$ and an elastic cylindrical annulus region. The graphs of $\tilde{Q}$ and $\tilde{r}$ versus $\Delta \tilde{P}$ are plotted with (a) $\tilde{p}_{0}=0$ and (b) $\tilde{p}_{0}=0.5$. 
The initial radius $r_{i}$ of the porous core is critical for this poroelastic model. $r_{i}$ represents the proportion of pores in the system and controls whether the applied pressure difference results mainly in flow or mainly in deformation. When $r_{i}=0$ and the pressure difference is small, the applied pressure mostly results in elastic deformation of the system and little flow throughout. Correspondingly, the flow rate increases slowly with applied pressure. Then, as $\Delta \tilde{p}$ becomes significant, the flow rate starts to rapidly increase, due to its $7^{\text {th }}$-degree polynomial relation with the pressure difference. When $r_{i}$ increases to $R$ or the whole tube is a pore, the model becomes a simple cylindrical empty tube, and we achieve a linear relation between flow rate and pressure difference as described by Poiseuille's law.

We developed this poroelastic model to investigate behaviors of a cellular aggregate under interstitial flow within the elastic limit of the cellular aggregate. The characteristic curve between pressure difference and flow rate will depend on the background pressure and the proportion of the porous behavior over elastic behavior.

\subsection{First microfluidic design to characterize interstitial flow with constant flow rates}

\subsubsection{Experimental setup and working principle}

\section{Experimental setup}

At the beginning, we verified the absence of leakage in each device by applying a constant flow rate of distilled water $(5 \mu \mathrm{lmin}-1)$ to the input channel, connecting channel 4 to the manometer, and closed all the other channels. We observed a linear increase of pressure in the manometer reading and stopped the flow when the pressure reached 
2000 Pa. The devices for which pressure remains constant after 15 minutes are considered as having no leakage and will be retained for the next step of experiments.

The microfluidic device used for experiments was placed inside a heating stage (Pecon heating Insert P Lab-Tek ${ }^{\mathrm{TM}} \mathrm{S} 1$ ) of an inverted fluorescent microscope (Axio Observer.Z1, Zeiss, Germany). The temperature of the stage is adjustable by a controller (Pecon TempController 2000-2). The images of the collagen gel chambers were taken by a high speed camera (Axiocam 506 Color, Zeiss, Germany).

In the experiments, the tubing connection follows the schematics in Figure 5.3. We used two digital manometers (HD 755-0.5 psi, Extech Instrument, USA) connected to channel 2 and 4 to monitor the pressure drop across the chamber in real time. These manometers have a piezoelectric sensor and are able to measure the pressure between $-3.447 \mathrm{kPa}$ to $3.447 \mathrm{kPa}$ with the resolution of $0.001 \mathrm{kPa}$ and the basic accuracy of $\pm 0.3 \%$ full scale. Channel 5 is connected to a reservoir of medium. Two syringe pumps (KDS Legato 210P, KD Scientific) were connected to channel 1 and 3, respectively. Syringe pump 1 was used to generate flow to the main channel, while syringe pump 2 was used to move a cellular aggregate into the chamber. Additionally, each syringe pump has a "shortcut" to the reservoir controlled by a tubing clamp. This configuration helped us move cellular aggregates into the chamber more easily and maintain a static pressure across all the channels at "no flow" condition. In detail, at no flow condition, K1 and K2 are unlocked so that all the channels are connected to the reservoir and stand the same static pressure. Note that the tubing clamp being locked means the tubing is disconnected. When moving the cellular aggregate into the chamber, $\mathrm{K} 1$ is locked and $\mathrm{K} 2$ is unlocked. When applying flow to the main channel, both $\mathrm{K} 1$ and $\mathrm{K} 2$ are locked. 


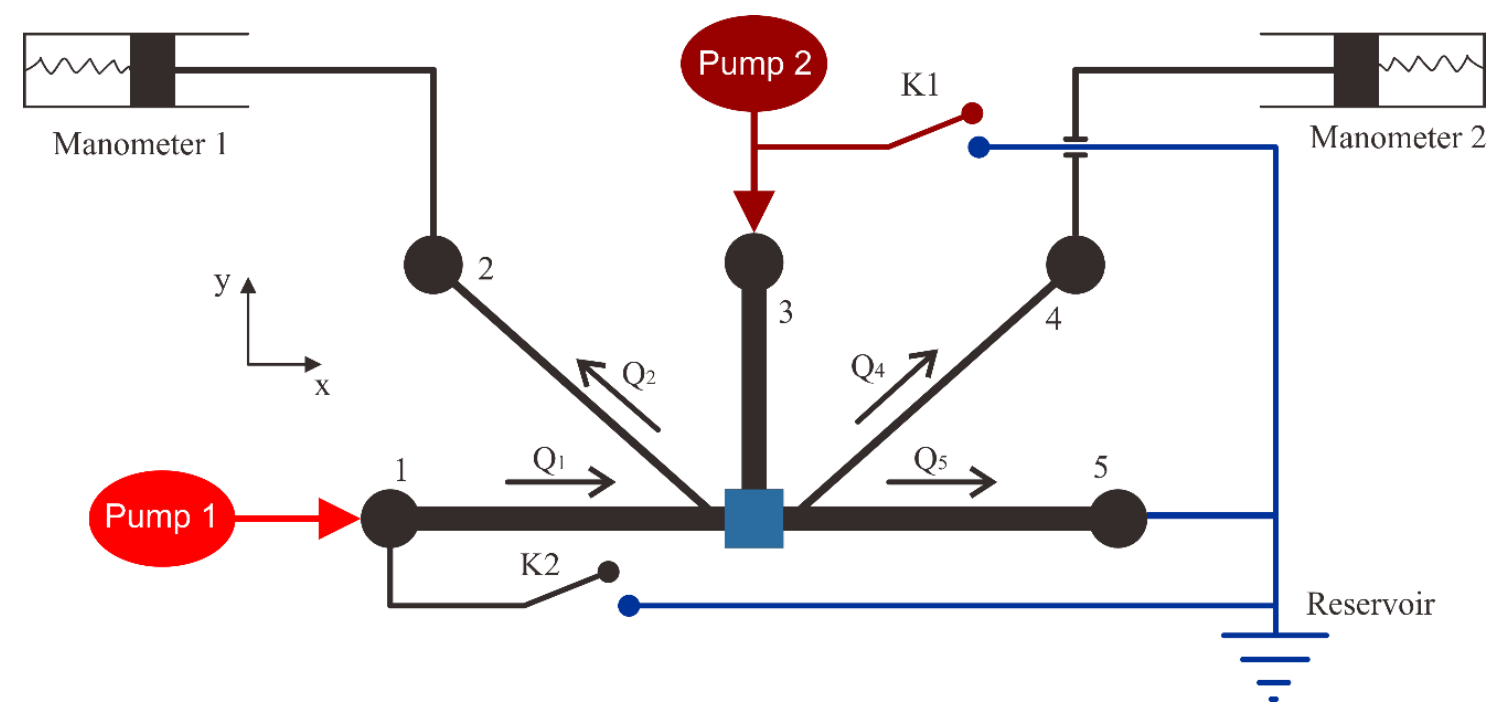

Figure 5.3 The schematics describes the working principle of the first design of microfluidic system to generate constant flow rates. K1 and K2 are the tubing clamps. K1 and K2 are unlocked for "no flow" condition. When using Pump 2 to move a cellular aggregate into the chamber, K1 is locked and K2 is unlocked. When applying flow through the main channel, both K1 and K2 are locked. Note: A 'locked' clamp means that the tubing is disconnected.

\section{Working principle}

This setup allowed us to characterize flow through a square cell chamber. In the first step, a cellular aggregate was moved to the chamber. We secured the aggregate inside the chamber by performing a surface coating of the microchannel, as discussed below. Then, flow would be applied through the main channel to the cell chamber. This flow caused a pressure difference across the cell chamber, which was measured by the manometers. At each run of the experiment, we applied a single constant input flow (resulting in a constant average flow velocity at the input channel) to the cell chamber.

When applying the flow, we first observed a transient regime where the pressure difference across the chamber increased over time. As the main channel was being mostly blocked by the cellular aggregate, the flow preferentially moved to channel 2 and made pressure in channel 2 side increase. When the flow kept moving to channel 2, the pressure here also kept on increasing, while the pressure in channel 4 and 5 was maintained by the 
reservoir. This caused the pressure difference across the cell chamber increasing, resulting in the increase of flow rate creeping through the cellular aggregate. Eventually, the flow rate of input flow was equal to the flow rate passing through the cell chamber, and thus the system reached a steady state, where the pressure reading remained constant over time.

\subsubsection{Calibration of the device with flow through an inert medium}

According to Darcy's Law, flow through a porous medium results in a linear relation between the flow rate and the pressure drop across the medium. Based on this theory, we verify our system by characterizing the flow through a chamber of type I collagen gel, a material which is known to behave as a porous medium. The gel permeability is also quantified using Darcy’s law equation.

\section{Preparation of type I collagen gel and Poly-D-Lysine (PDL) coating}

For the calibration tests, we coated the microchannels with Poly-D-Lysine solution at $1 \mathrm{mg} \mathrm{ml}^{-1}$ (PDL, Sigma Aldrich, Singapore). $50 \mu 1$ of PDL solution was injected to the microchannels. The devices were then placed inside a $37^{\circ} \mathrm{C}$ incubator for more than 4 hours. After the coating, we aspirated the PDL solution and washed the devices 3 times with distilled water. The devices were put in an oven at $80{ }^{\circ} \mathrm{C}$ for 24 hours for PDMS surface hydrophobicity restoration [40]. This step helped preventing the injected gel solution from wetting and spreading out of the chamber.

We prepared two batches of bovine type I collagen gel solution (cat\# A10644-01, Thermo Fisher Scientific, Singapore) at two different concentrations: $2 \mathrm{mg} \mathrm{ml}^{-1}$ and $3 \mathrm{mg} \mathrm{ml}^{-1}$ (see Appendix 1). The gel solutions are then mixed with $2 \mu \mathrm{l}$ of FITC fluorescein dye solution $\left(1 \mathrm{mg} \mathrm{ml}^{-1}\right)$ to image the gel shape formed inside the microfluidic device. We introduced the gel solution to the central chamber of our device by 
micropipetting through channel 3. Thanks to the pillars placed inside the central chamber, the gel solution was contained in the chamber by its surface tension. Channel 3 was then clamped to prevent the gel flowing and to hold the gel securely inside the chamber. The device containing the gel solution was placed inside a $\mathrm{CO}_{2}$ incubator at $37^{\circ} \mathrm{C}$. The gel resulted in a rigid chamber-shaped region after 30 minutes of incubation (Figure 5.4a).

\section{Calibration run}

When running the calibration, the microscope stage was set at $37^{\circ} \mathrm{C}$. We started the calibration by applying an input flow with constant average velocity to the gel chamber. The pressure difference across the gel chamber was observed to increase to a constant value at the first steady state (Figure 5.4b). When the pressure reached a constant value, we moved to a higher value of the input flow rate, in order to acquire a new steadystate value of the pressure difference at this higher flow level. We name this protocol "stepping flow rates". During the calibration, we used distilled water as the fluid flow. A stepping flow rate from 0 to $700 \mathrm{nl} \mathrm{min}^{-1}$, with each step of $100 \mathrm{nl} \mathrm{min}-1$, was applied to the main channel. As expected, the pressure difference collected at each input flow rate is linearly proportional to the imposed flow rate (Figure 5.4c). This confirms that the gel behaves as a porous medium and thus supports the reliability of our system to characterize porous media flow.

From the slope of the pressure-flow relationship, we quantified the gel permeability through experiment data. The collagen gel permeability in our experiments ranges from $1.6 \times 10^{-13}$ to $5 \times 10^{-13} \mathrm{~m}^{2}$ for different batches of gels, a variability that can be attributed to differences in the gelification process. Our average permeability value agrees with previous studies on the permeability of this type of gel, thus confirming the validity of our technique [38, 44, 178]. 
$\mathbf{a}$
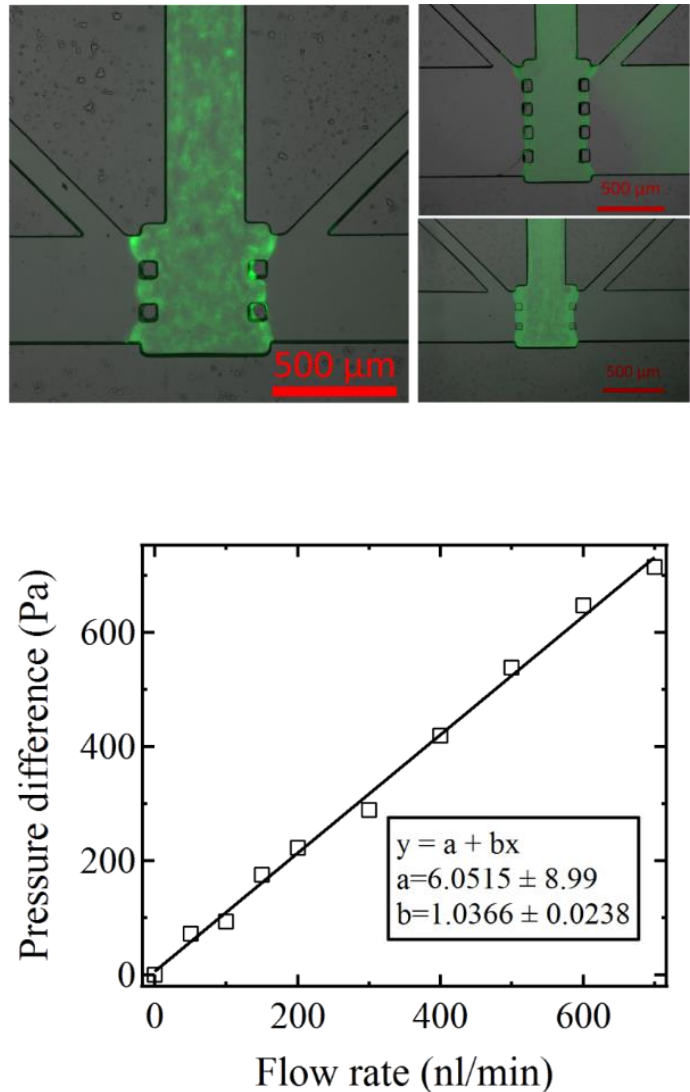
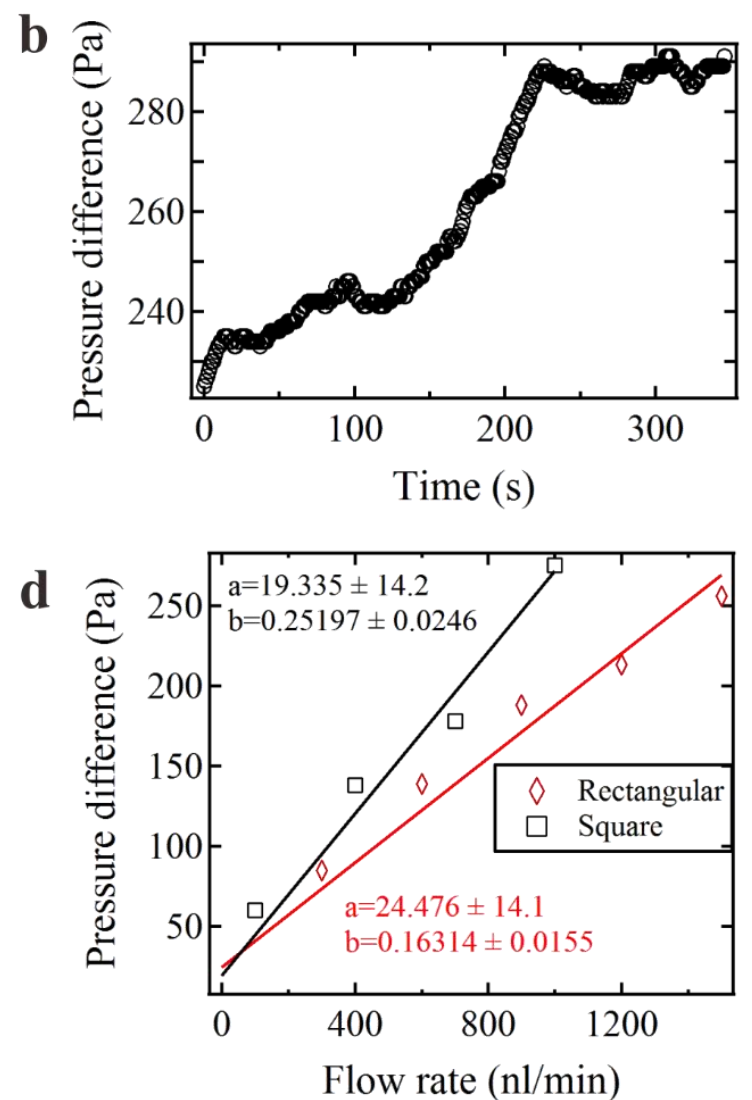

Figure 5.4 Experimental results of device calibration for the first design with type I collagen gel. (a) The images of collagen gel rigidified inside the chamber, (left) the gel in square chamber is made from $3 \mathrm{mg} \mathrm{ml}^{-1}$ gel solution; (top right) the $2 \mathrm{mg} \mathrm{ml}^{-1}$ gel solution was shaped in rectangular chamber; (bottom right) the $2 \mathrm{mg} \mathrm{ml}^{-1}$ gel solution was shaped in square chamber; scale bar is $500 \mu \mathrm{m}$. (b) The increase of pressure difference to a constant value at steady state when a constant flow rate of $150 \mathrm{nl} \mathrm{min}^{-1}$ was applied through a $3 \mathrm{mg} \mathrm{ml}^{-1}$ square gel chamber (c) The linear relation between pressure difference and flow rate across the $3 \mathrm{mg} \mathrm{ml}^{-1}$ gel chamber at each steady state, showing the hydrodynamic resistance of the collagen gel. From here, using Darcy's law equation, we calculate the permeability of this collagen gel is $1.62 \times 10^{-13} \mathrm{~m}^{2}$. (d) The comparison of hydrodynamic resistance between collagen gel in square shape $(500 \mu \mathrm{m} \times 500 \mu \mathrm{m})$ and rectangle shape $(500 \mu \mathrm{m} \times 1000 \mu \mathrm{m})$. Two different shapes of gel resulted in 2 different resistance values, but the permeability is the same due to the same batch of $2 \mathrm{mg} \mathrm{ml}^{-1}$ gel $\left(6.11 \times 10^{-13} \mathrm{~m}^{2}\right.$ and $5.17 \times 10^{-13} \mathrm{~m}^{2}$, respectively). Note: the fitting curves in the graph have a linear function of $y=a+b x$.

In a different experiment, we wanted to test if the shape of gel region affects the gel permeability in the same batch of gel. We made a batch of collagen gel, and inject it into two devices which have two different shapes of gel region (Figure 5.4d). All the devices exhibited a linear relation between flow rate and pressure difference and also resulted in virtually identical values of gel permeability, thus indicating that our method is robust and sufficiently insensitive to details of the geometry of the device. The experiments with collagen gel allowed us confirming the reliability, repeatability and feasibility of our system. 


\subsubsection{First experiment with cellular aggregates}

After having successfully described flow through an inert porous medium using Darcy's law, we next moved to experiments with cellular aggregates.

\section{Preparation}

Before starting the cell aggregate experiment, we coated the microchannels with bovine fibronectin solution (F1141, Sigma Aldrich, Singapore) at a concentration of $100 \mu \mathrm{g} \mathrm{ml}^{-1}$. The microchannels were left to incubate at $37^{\circ} \mathrm{C}$ with fibronectin for at least 4 hours. The device was then washed with PBS solution and ready for the experiment. Additionally, we conducted a simple test to verify the quality of the fibronectin coating layer on the microchannel surfaces. By repeating the same coating procedure, we coated a batch of microchannels with a solution of bovine fibronectin conjugated with fluorescent HiLyte488 (FNR02-A, Cytoskeleton, Denver, USA) and compared with another batch of microchannels coated with only FITC fluorescein dye in DMSO solution. All the microchannels were dried in an oven at $37{ }^{\circ} \mathrm{C}$ for 2 hours before observation.

At the same observation conditions (Reflector: AF488, exposure time: $300 \mathrm{~ms}$ ), the fluorescent fibronectin coated-microchannels displayed a bright uniform layer of green fluorescein, while the FITC coated-microchannels resulted in weak fluorescent intensity. We also quantitatively measured the fluorescent intensity of the coated microchannels by ImageJ software. The measurement was taken at 5 regions (Figure 5.5). The average mean gray value of the background was $\sim 5$, while that of the fluorescent fibronectin coated-regions and FITC coated-regions was 32.0656 and 8.1195, respectively. Thus, the fluorescent fibronectin regions showed a quantitatively higher fluorescent intensity. This revealed that the presence of fibronectin helped the fluorescein 
remain better on the microchannel surfaces. It also confirmed the feasibility of the fibronectin coating.
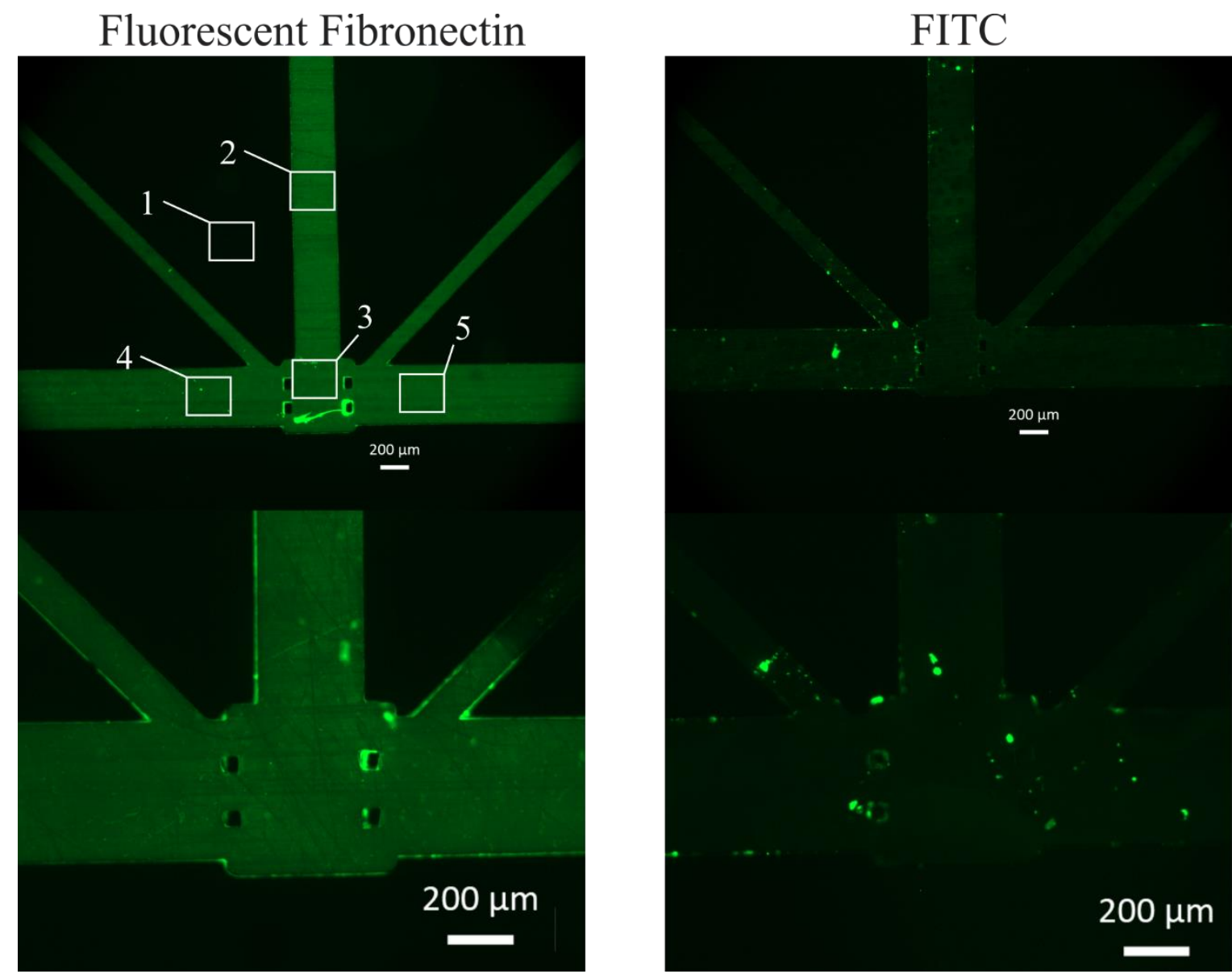

Figure 5.5 The fibronectin coating quality test. Fluorescent intensity was measured at 5 regions using ImageJ (Region 1 is the non-fluorescent background). The microchannels coated with a fluorescent fibronectin solution (bovine fibronectin conjugated with HiLyte488) (left) showed the higher fluorescent intensity than the microchannels only coated with FITC fluorescein dye solution (right). This result revealed the appearance of fibronectin made the fluorescein dye remain in the microchannel surfaces. Scale bar: $200 \mu \mathrm{m}$.

\section{Moving a cellular aggregate into the cell chamber}

In the next step, we placed the coated device inside the microscope stage and filled the microchannels with $\mathrm{CO}_{2}$-equillibrated culture medium. Then, we connected the tubing as required for the experiment (tubing connection is represented in Figure 5.3). In order to apply flow through the cellular aggregate, we first need to move the aggregate to occupy the central chamber. Acknowledging that tissue behaves analogously to a viscoelastic 
liquid $[134,179]$, we plan to apply flow to make the cell aggregate squeeze through channel 3 and enter the chamber.
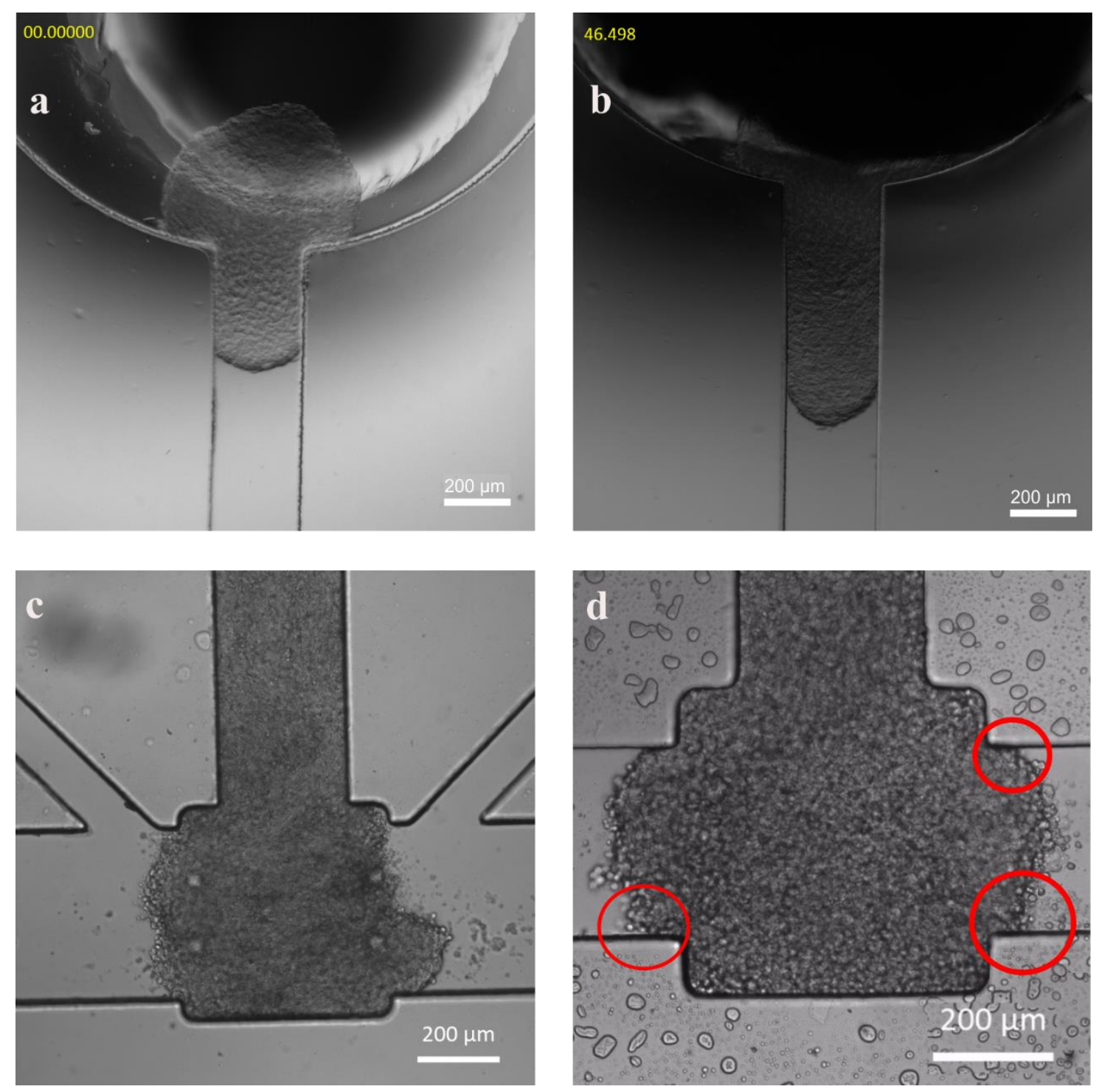

Figure 5.6 A cellular aggregate moved into the central chamber. (a) The cellular aggregate was partially dragged into channel 3. (b) The cellular aggregate was slowly pushed to move inside channel 3. (c) The aggregate reached the chamber. (d) After the heating stage was set at $37^{\circ} \mathrm{C}$, the aggregate adhered to channel walls. The red circles are the signs of the cells spreading onto the channel walls.

First, we used a pipette to inject a selected cellular aggregate into the inlet of channel 3. Next, we connected the inlet of channel 3 to syringe pump 2 and locked the tubing clamp K1 (while K2 was unlocked, see Figure 5.3). At this time, the microscope stage was kept at room temperature $\left(23-25^{\circ} \mathrm{C}\right)$ to prevent cells from adhering to the walls of channel 3. We applied an arbitrary flow rate then stopped the flow when the front 
of the cell aggregate was dragged into channel 3. From here, we slowly applied flow to push the cell aggregate move into the chamber. The detailed procedure and setup for moving the cell aggregate into the chamber is as follows:

1) During this session, the heating stage of the microscope, where the microfluidic device is placed inside, need to be off. The temperature of the device is at room temperature $\left(23-25{ }^{\circ} \mathrm{C}\right)$. All the microchannels need to be pre-filled with $\mathrm{CO}_{2}$ equilibrated culture medium.

Note: It is critical to make sure there is no bubbles inside the microchannels.

2) We used two scissor clamps (with flat jaws) to clamp manometer tubing at channel 2 and 4 . This helps the flow not going through the manometer channels, which can result in the manometer malfunction due to exceeding pressure.

3) Unplug the tubing of Pump 2 in channel 3 inlet off the PDMS device, leaving an open inlet in channel 3.

4) A selected cellular aggregate with diameter of $400-500 \mu \mathrm{m}$ is injected to channel 3 inlet by pipetting. Then re-plug the tubing of Pump 2 to channel 3 inlet.

5) At this point, tubing clamp $\mathrm{K} 1$ is locked and $\mathrm{K} 2$ is unlocked. Turn on the live imaging of the microscope to observe the aggregate moving session in real time. We will need to control the syringe pump while observing the cellular aggregate moving inside the microchannel.

6) We start to apply an arbitrary flow rate from Pump 2 to make the cellular aggregate move to the junction between the inlet and the microchannel 3 (Figure 5.6a). When the cellular aggregate partially enters the channel, quickly stop the syringe pump. According to our own microchannel size (the inlet of channel 3 
with diameter of $2 \mathrm{~mm}$ and channel 3 with diameter of $300 \mu \mathrm{m}$ ), we applied a flow rate at $100-200 \mu 1 \mathrm{~min}^{-1}$ for this step.

Note: If the flow is not stopped in time when the cellular aggregate enters the channel, it will burst the cellular aggregate. In case that the syringe pump used cannot function to stop that quickly, we suggest replacing tubing clamp K1 for a scissor clamp. Before applying the flow, the scissor clamp K1 is locked. When the cellular aggregate is partially inside the channel 3, quickly release the scissor clamp K1. This action will make flow from Pump 2 go directly to the reservoir without pushing the cellular aggregate any further.

7) Continue to apply a flow rate of $10-20 \mu 1 \mathrm{~min}^{-1}$ and observe the cellular aggregate moving inside the microchannel (Figure 5.6b). Keep the cellular aggregate move with a steady speed by turning the pump on and off continuously.

8) When the cellular aggregate reaches the chamber, stop the pump. Let the cellular aggregate recover and expand to all the chamber area (Figure 5.6c, d).

9) Release the clamps at the two manometer tubing.

After moving the cell aggregate, the microscope stage is adjusted to $37^{\circ} \mathrm{C}$ and both tubing clamp K1 and K2 were unlocked to create a static condition for the cellular aggregate. The aggregate was left to "relax" inside the chamber. As investigated in the study of Douezan et al. [137], the cellular aggregate would spread onto a rigid PDMS surface due to its wetting behaviors over a typical time scale of one hour. This would help the cellular aggregate adhere to the channel wall with Fibronectin coating. In our experiment, the periphery of the aggregate was observed to adhere to the channel walls after about one hour. The adhesion could also be recognized by the change of cell color in focus plane (the adhering cells become darker in Figure 5.6d). Moreover, we verified that 
firm adhesion to the channel walls was attained after about one hour. Indeed, after onehour spreading, our aggregates were rarely detached from the wall by fluid flow forces, and no preferential flow leakage between the aggregate and the wall was observed in fluorescein flow experiments. For these reasons, we set a fixed time of 2 hours as the amount of time for every cellular aggregate to "relax" and conduct the adhesion to the channel walls. After the "relaxation" period, we started to apply flow through the cell aggregate. The fluid consisted of $\mathrm{CO}_{2}$-equillibrated culture medium mixed with $1 \%$ of FITC fluorescein dye to help observing how the flow went through the cell aggregate. The manometers connected to channel 2 and 4 helped monitoring the pressure difference across the cell chamber.

\section{Results}

The cellular aggregate adhered to the chamber and formed a 3D aggregate in rectangular cuboid of dimensions $500 \times 500 \times 110 \mu \mathrm{m}$. We also began the cellular aggregate experiment by applying a constant flow input to the main channel. Similar to the collagen gel experiment, at steady state the pressure difference reached a constant value (Figure 5.7b). At steady state, the flow was observed to creep through the cell aggregate to the output channel by creating a fracture inside the cell aggregate (Figure 5.7a). This flow-induced fracture is indicative of the cellular aggregate behaving as a fragile solid.

We further investigated the fracture behavior of the cellular aggregate by applying multiple constant average flow inputs, stepping from 0 to $8 \mu \mathrm{m} \mathrm{s}^{-1}$ (with $0.5-1 \mu \mathrm{m} \mathrm{s}^{-1}$ increasing at each step). At each flow input value, we recorded the steady-state pressure difference. The combined result shows that, as the flow input to the cell aggregate increased, the pressure difference across the cell chamber first increased up to a 
maximum. Further increase of the imposed flow rate resulted in a rapid decrease of pressure difference with increasing flow (Figure 5.7c). Based on this result, we hypothesized that the behavior of the flow might be classified in two regimes:

- For an input flow rate between 0 and $2 \mu \mathrm{m} \mathrm{s}^{-1}$, pressure difference increased with flow rate. These experimental results are consistent with the aggregate behaving as a porous medium following Darcy's law, or possibly as a poroelastic material.

- When the flow input became higher than $2 \mu \mathrm{m} \mathrm{s}^{-1}$, pressure difference decreased with increasing flow rate. This corresponds to irreversible aggregate fracture, which can be described as plastic solid behavior.

At the transition between these two regimes, there may be a "breaking point" of the cell aggregate corresponding to the maximum pressure difference and to the onset of fracture. This observation may have important physiological consequences. Indeed, if interstitial flow can induce fracture happened inside a tumor, the damaged part of the tumor may lead to vast detachment of cells from the tumor and increase the risk of metastasis. Thus, the question of whether and how interstitial flow fractures a tumor appears crucial.

The theoretical model in 5.1 can be used to model the first regime of the aggregate behaviors under the flow before the "breaking point". Experimentally, however, our current observations show a linear relationship between flow rate and applied pressure difference, a behavior that can be well described by the simpler Darcy's law. In the context of the poroelastic model, our experiments correspond to a regime where an applied pressure finds it easier to generate a flow rather than to induce an elastic deformation of the aggregate. 
a
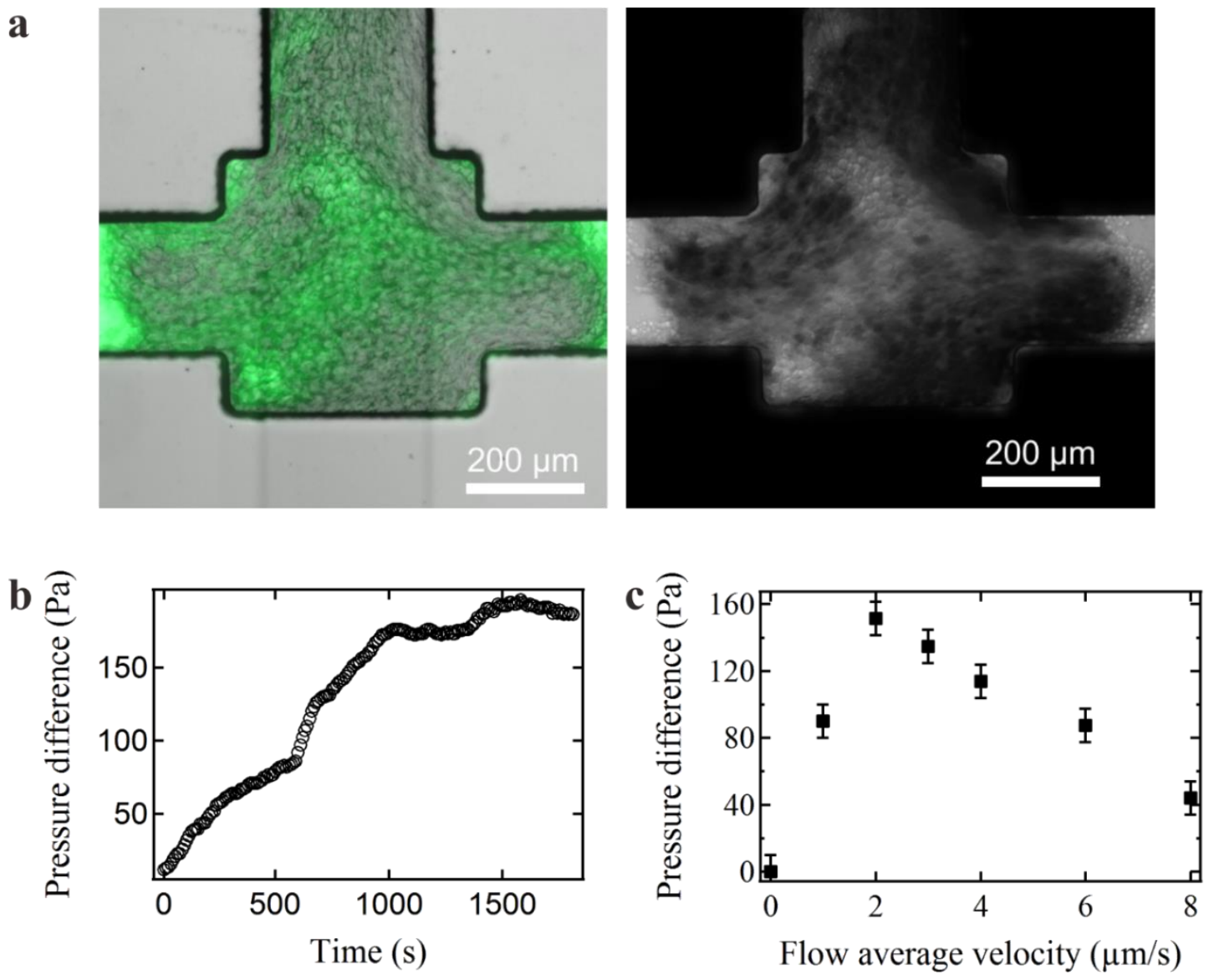

Figure 5.7 Experimental results with cell aggregates (a) An image captured from the movie of flow through a MCF-7 cellular aggregate inside the cell chamber, right after fracture occurred. The flow with fluorescein was observed to advance progressively and very slowly from the left side towards the right side and then, suddenly, to reach all the way to the output (b) The increase of pressure across the cell aggregate when we applied flow at $4 \mu \mathrm{m} \mathrm{s}^{-1}$. The pressure achieved a constant value when the system reached the steady state. (c) The pressure difference across the cellular aggregate chamber increased to a highest value then dropped when the flow velocity kept increasing. The error bars are standard deviations. We hypothesize that the cellular aggregate behaves as two regimes: poroelasticity and solid plasticity. These two regimes are separated by a "breaking point" when the fracture happened inside the cellular aggregate.

\subsubsection{Limitation of the microfluidic device for interstitial flow with constant flow rate}

From this point of the study, our goal became to understand how the occurrence of aggregate fracture depended on the fluid mechanical parameters of the interstitial flow attempting to traverse the aggregate. We have identified two key questions: (1) to determine the critical flow rate that induces aggregate fracture, which is a measure of the hydrodynamic load applied on the aggregate, and (2) how this critical flow rate depends on the loading rate at which the flow is applied. In conducting this second part of the 
study, we realized some shortcomings of the current microfluidic system model that might interfere with our aggregate fracture investigations.

In the first design, the time required to reach hydrodynamic steady state can range from 30 minutes to more than one hour depending on the flow rates. This delay time required each flow rate to run constantly for a certain period of time before moving to a new flow rate. As a result, we could only increase the flow rate "step by step" in the previous experiments. Thus, it might not be feasible to investigate the influences of different loading rates of the flow acting on the cellular aggregates.
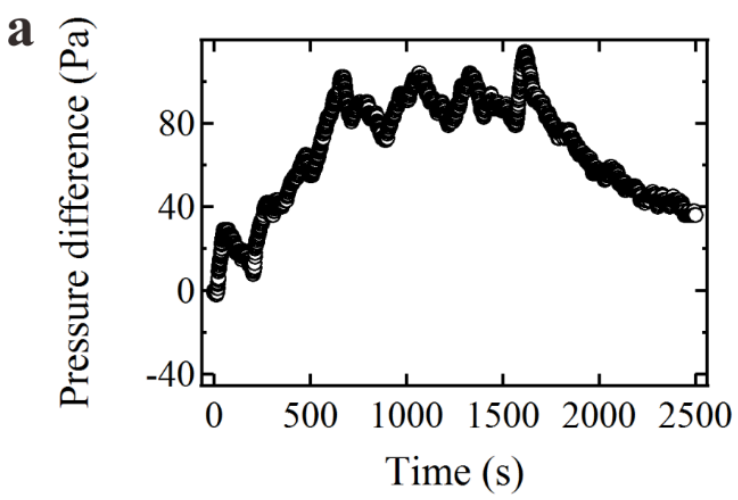

C

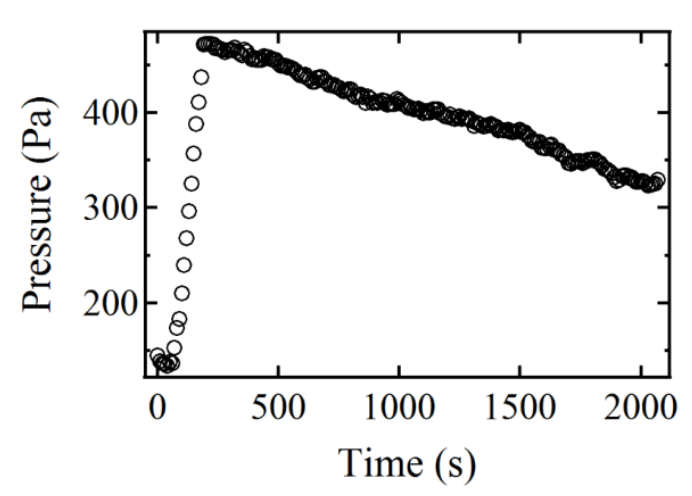

b

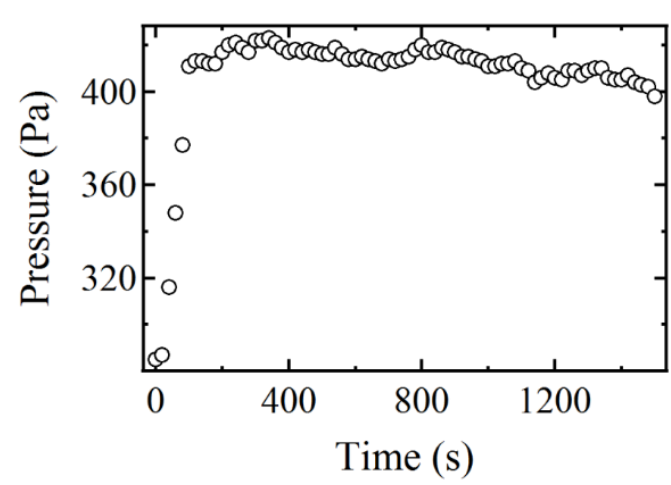

d

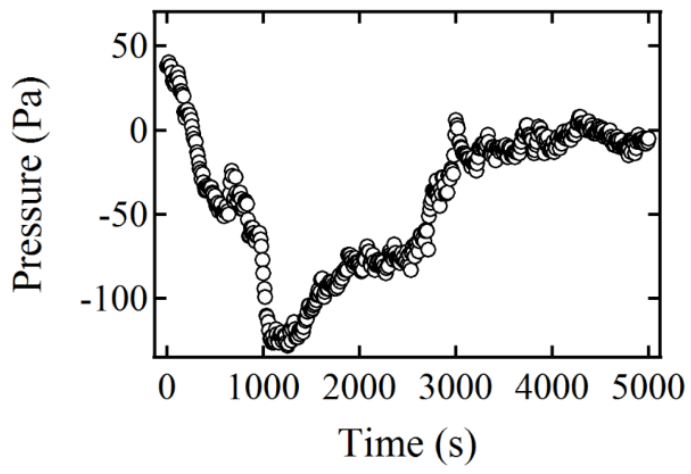

Figure 5.8 The experimental results showing the problem with unstable pressure readings at low flow velocity. (a) The pressure difference was observed to drop after 1600 seconds at flow velocity of $0.2 \mu \mathrm{m} \mathrm{s}^{-1}$. (b) A test run with water inside a closed microchannel system, at $25^{\circ} \mathrm{C}$. The pressure was constant for 1600 seconds. (c) A test run with water inside a closed microchannel system, at $37^{\circ} \mathrm{C}$. The pressure was observed to drop linearly. (d) A test run with water inside a closed microchannel system, at $37^{\circ} \mathrm{C}$. The device was then submerged in distilled water and the pressure was observed to increase to a constant value.

Furthermore, when applying a low input flow velocity in range of 0 to $1 \mu \mathrm{m} \mathrm{s}^{-1}$, we faced a problem of unstable pressure readings from the manometer. At flow input velocity 
of $0.2 \mu \mathrm{m} \mathrm{s}^{-1}$ we observed that the pressure difference started to drop after 30 minutes while the flow was still being applied (Figure 5.8a). This could be due to the evaporation of culture medium through PDMS, a problem that has been reported in some previous studies $[180,181]$. In our first design, when the cell aggregate enters the central chamber, the fluid medium is separated into two regions initially isolated from each other. The input channel (channel 1) becomes a closed channel with only fluid flowing from the syringe pump, while the output channel is open to a reservoir of culture medium. Thus, a differential medium evaporation at $37^{\circ} \mathrm{C}$ between the two regions results in an unstable pressure built up at two sides of the cell chamber.

We have run a test to investigate the evaporation problem. The goal of the test was to check whether the manometer readings remained consistent in the presence of evaporation. We set up the test by using our microfluidic device with one inlet connected to the syringe pump and another inlet connected to the manometer while the other inlets were blocked. First, we pumped an amount of distilled water into the microchannels so that the pressure in the channels rose up to $\sim 400 \mathrm{~Pa}$, then we stopped the pump and observed the pressure readings, which we expected to either remain constant, if evaporation is negligible, or to linearly decrease in time, if evaporation is present. At this time, we left the setup at room temperature. The result showed that after 1600 seconds, the pressure was constant within the accuracy range of the manometer, indicating that evaporation is negligible at room temperature (Figure 5.8b). In the second run, we repeated the first run but heating up the setup to $37^{\circ} \mathrm{C}$, as needed for the cell aggregate experiment. The pressure was observed to drop significantly, suggesting evaporation (Figure 5.8c). Note that, the second run was carried out right after the first run. We then conduct the third test run by submerging the microfluidic device inside a petri-dish of distilled water while the temperature of the platform was still $37^{\circ} \mathrm{C}$. After decreasing for 
1000 seconds, the pressure recovered and became stable (Figure 5.8d). The third test run has given us the evidence of the water evaporation was involved in the experiments. The evaporation was avoided by submerging the microfluidic device in water.

This test has revealed that our first experiment was affected by water evaporation. However, these three test runs were conducted in a device with empty microchannels. While having a cellular aggregate inside the chamber of the device, the submerging the device in water could reduce the evaporation, but the problem of differential evaporation between two fluid regions (upstream and downstream of the cellular aggregate) would still interfere the manometer readings.

From our tests, we conclude that evaporation can be neglected when the imposed flow velocity was higher than $1 \mu \mathrm{m} \mathrm{s}^{-1}$, since in this range the pump-induced flow rate is significantly higher than the evaporation rate, which can thus be neglected. However, at lower flow velocity, the evaporation rate cannot be neglected and significantly affects the effective flow rate. We note that the presence of evaporation does not invalidate our finding of two regimes of cellular aggregate mechanical behavior, although it does affect the value of the permeability deduced in our previous experiments.

In the next part, we introduced the second design to characterize interstitial flow with increasing flow rates. The second design no longer studied the porous/poroelastic regime corresponding to low flow conditions, but rather the higher flow conditions that induce aggregate fracture. 


\subsection{Second microfluidic design to characterize interstitial flow with continuously increasing flow rate}

\subsubsection{The advantages of the second design}

We introduce the second microfluidic design to characterize the interstitial flow with continuously increasing flow rate. The goal of this part of the study is not to investigate poroelasticity of the cellular aggregate, but the occurrence of aggregate fracture, for which the magnitude of interest is the critical pressure difference at which fracture occurs.

In the second design, by introducing the new side channel that permanently connect the input and output channels to each other, we have prevented differential evaporation even when the cell aggregate is present in the cell chamber. The medium from the reservoir of the outlet is also able to refill the input flow channel in case no flow or slow flow velocity is applied. The other advantage of this new design compared to the first design is that the side channel can be used to measure the pressure difference across the cell chamber, thus potentially replacing the manometer.

Another advantage of the second design is that the pressure difference response to flow velocity changes will be much faster than the previous design, thus overcoming the long wait to reach steady state needed in the first design.

\subsubsection{Calibration of the new device}

We carried out the calibration of the new device to confirm that the side channel has the intended hydrodynamic resistance. First, we managed to introduce a small amount of PDMS solution with curing agent (ratio of PDMS monomer and curing agent is 10:1) into the cell chamber. When getting cured, this PDMS portion became a blockage that stopped the flow from moving through the cell chamber (see Appendix 2 for more detail). As a result, the flow applied to the inlet only moved through the side channel. The pressure 
difference measured in this setup corresponds to the pressure difference across the side channel, and the flow rate imposed goes fully through the side channel. Thus, we were able to quantify the hydrodynamic resistance of the side channel.

As shown in equation (3.2), the resistance depends on the viscosity of the fluid flow. We ran the calibration tests with the culture medium at $37^{\circ} \mathrm{C}$ so that it corresponds to the actual conditions with cell aggregates. Several types of flow were applied to the side channel: constant flow rate, stepping flow rates, and linear increasing flow rates with different accelerations. These calibration runs (Figure 5.9) result in a consistent value of hydrodynamic resistance of the side channel of $32.18 \pm 0.28 \mathrm{~Pa} \mathrm{~min}_{\mu l^{-1}}$ (The error is calculated from standard deviation of 5 calibration runs). This resistance will be used as the reference to quantify the pressure difference of the cell chamber.
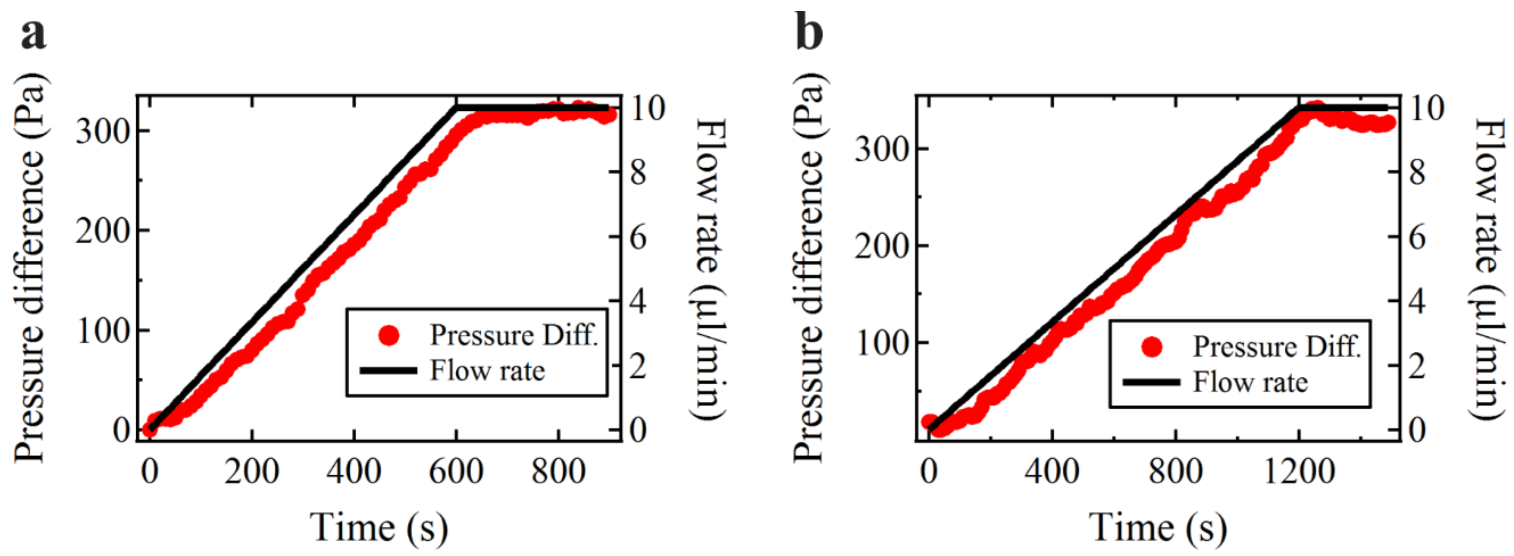

Figure 5.9 The graph shows the calibration results when applying linear increasing flow rate at (a) loading rate 1 with increase rate of $1 \mu 1 \mathrm{~min}^{-2}$ and (b) loading rate 2 with increase rate of $0.5 \mu 1 \mathrm{~min}^{-2}$. The hydrodynamic resistance of the side channel to culture medium was measured and calculated at 32.18 Pa $\min \mu 1^{-1}$.

The calibration tests have proven the validity and flexibility of this new design. In order to assure that side channel dimensions are constant across all experiments, we produced all duplicate devices from one single mold pattern, corresponding to the calibration above. 


\subsubsection{Second experiment with cell aggregates}

\section{Preparation}

We used the devices that are duplicates of the calibrated device to carry out experiments with cell aggregates. In these experiments, we also coated the device with fibronectin solution $\left(100 \mu \mathrm{g} \mathrm{ml}^{-1}\right)$ and filled the microchannels with $\mathrm{CO}_{2}$-equilibrated culture medium, following an identical protocol as the one described above. The experiment was also started by moving a cell aggregate into the chamber (Figure 5.10a).

\section{Results}

First, we imposed an input flow rate that linearly increases with time and collected the data of pressure difference across the cell chamber When the flow increased, the pressure difference also increased linearly until the fracture happened inside the cellular aggregate (Figure 5.10b).

The pressure difference then dropped to a much lower value while the flow rate keeps increasing. We plotted the pressure difference and flow rate on Figure 5.10c. The hydrodynamic resistance of the system formed by the cellular aggregate in parallel with the side channel has an initial constant value of $\sim 30 \mathrm{~Pa}^{\mathrm{min}} \mu \mathrm{l}^{-1}$, slightly lower than that of the side channel alone. This equivalent resistance dropped to a lower value of $\sim 5 \mathrm{~Pa} \min \mu 1^{-1}$. This sudden drop of the equivalent resistance is also a sign to recognize the appearance of the aggregate fracture, and indicates that after fracture, the flow preferentially goes through the fractured aggregate, whose equivalent resistance is thus on the order of $5 \mathrm{~Pa} \min \mu \mathrm{l}^{-1}$. 

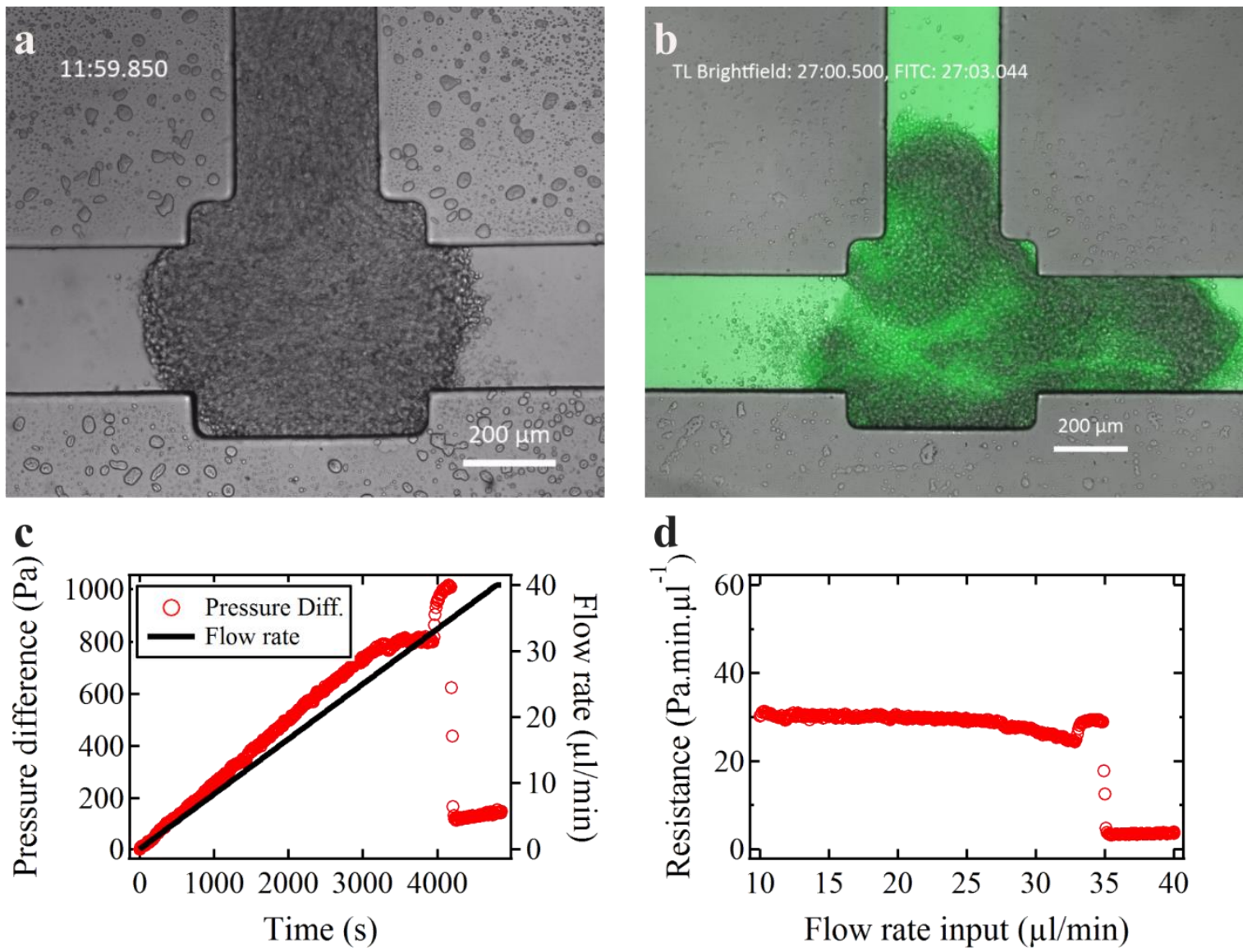

Figure 5.10 Experimental results with cellular aggregates in the second design. (a) The image of the cellular aggregate sitting inside the chamber at $37^{\circ} \mathrm{C}$. (b) The fracture happened inside the cellular aggregate when the flow was applied. (c) The graph shows how the pressure difference changed over time while increasing the flow rate. (d) The equivalent resistance of the cellular aggregate parallel with the side channel was calculated. The figure shows a sudden drop in resistance when fracture occurs.

In the next test, taking advantage of the ability of our device to modulate the flow profile, we investigated whether the critical pressure at which aggregate fracture occurs depends on the rate at which the input flow rate is ramped up. This is an important question, since it has been shown that, same as polymer melts, the mechanical response of a cellular aggregate to a force depends on the loading rate at which the force is applied [138]. By applying flow to cell aggregates with two different loading rates, we have observed that a higher rate of increase of the input flow rate resulted in higher pressure at aggregate fracture (Figure 5.11). This experiment has been carried out with 5 cellular aggregates in the same batch for each loading rate. This result suggests a new perspective on the effects of time-dependent flow on a tumor. The occurrence of tumor 
fracture induces a high risk of cancer metastasis. Our results suggest that the potential occurrence of aggregate fracture may depend not only on the magnitude of the flow rate, but also in the time-dependence of the flow profile.

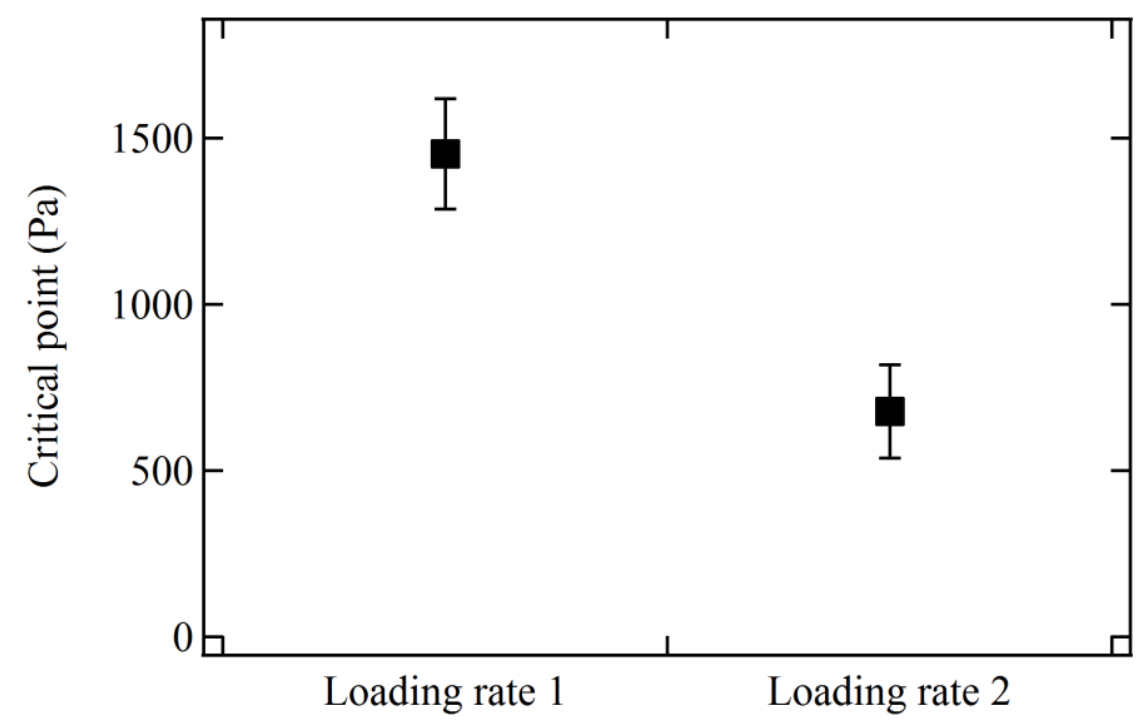

Figure 5.11 The graph shows the breaking points of cellular aggregates at two different loading rates. Loading rate 1 at $1 \mu 1 \mathrm{~min}^{-2}$, loading rate 2 at $0.5 \mu 1 \mathrm{~min}^{-2}$. Each loading rate was run with 5 cellular aggregates of the same batch. The error bars are the standard deviations.

\subsection{Discussion}

\subsubsection{Simple microfluidic setup for interstitial flow characterization}

In this study, we have introduced a simple microfluidic platform to characterize interstitial flow through a model tumor. Despite its shortcomings arising from evaporation in the PDMS device, the first design allows us to generate flow through a collagen gel matrix and a cellular aggregate, as long as the flow velocity is higher than $1 \mu \mathrm{m} \mathrm{s}^{-1}$. This setup can be used to quantify hydrogel permeability or to study the effects of interstitial flow on tumor cells embedded in a collagen gel chamber. In the experiments with cellular aggregates, the first design allowed us to observe that interstitial flow can induce cell 
aggregate fracture. These observations suggested the hypothesis of a fracture threshold that separates two regimes of aggregate behavior: poroelasticity and solid plasticity.

The second microfluidic design has resolved the problems of the first design and allows a quantitative study of aggregate fracture, and specifically how the fracture conditions depend on the characteristics of a time-dependent flow rate.

Our microfluidic devices provide simple experimental setups to study in vitro interstitial flow. The time scale for each experiment run is within 1-2 hours, which is much less than other microfluidic assays on interstitial flow $[45,52,58,182]$. By producing the cellular aggregates outside the setup and transferring them to the microfluidic devices, we saved time by not having to wait for the cells to grow inside the devices. Since cellular aggregates can adhere firmly to the microchannel walls coated with extracellular matrix proteins, they are a suitable model system for microfluidic assays.

\subsubsection{Behaviors of cellular aggregates under interstitial flow}

The experimental results have shown that, when exposed under interstitial flow, cellular aggregates experience two regimes of material behavior with a transient point. When the flow velocity increases, leading to the increase of pressure difference across the aggregate, cellular aggregates first behave as porous (or potentially poroelastic) materials. In this regime, the correlation between pressure difference and flow velocity follows a linear relation that can be characterized by Darcy's law. This regime exists until the pressure difference reaches a breaking point. At this point, fractures happen inside the cellular aggregates, resulting in a sudden drop of pressure while the flow velocity is still increasing. In contrast with the low-velocity regime, where aggregates behave as porous 
media, aggregate fracture corresponds to the cellular aggregates behaving as fragile solids.

We emphasize the fact that, from a biomedical standpoint, tumor fracture can induce the detachment of many cells from the tumor, and thus be a key mechanism to induce cancer metastasis.

\subsubsection{The dependence of cellular aggregate behavior on the flow loading rate}

We have observed that different loading rates of the flow input velocity result in different critical pressures for the cell aggregate to fracture. Specifically, a higher loading rate yields a higher critical pressure at fracture. This can be explained based on a viscoelastic behavior of cellular aggregates, characterized by a typical deformation time defined by the ratio of viscosity to elasticity. At high loading rate, flow evolves rapidly compared to this characteristic viscoelastic time, and aggregates have little time to deform while pressure increases up to fracture-inducing levels. This causes the fracture to occur at a higher pressure but in a shorter time. At low loading rate, we hypothesize that flow increases slowly compared to the viscoelastic time, and aggregates have time to deform (Figure 5.12). Thus, cellular aggregates are deformed more slowly and can deform more before fracture, suggesting a ductile fracture for slow loading as opposed to a brittle fracture for fast loading. Since for low loading rate aggregates have a longer time to deform, flow-induced deformation could weaken the intercellular bonds inside cellular aggregates, resulting in a lower pressure at fracture. 

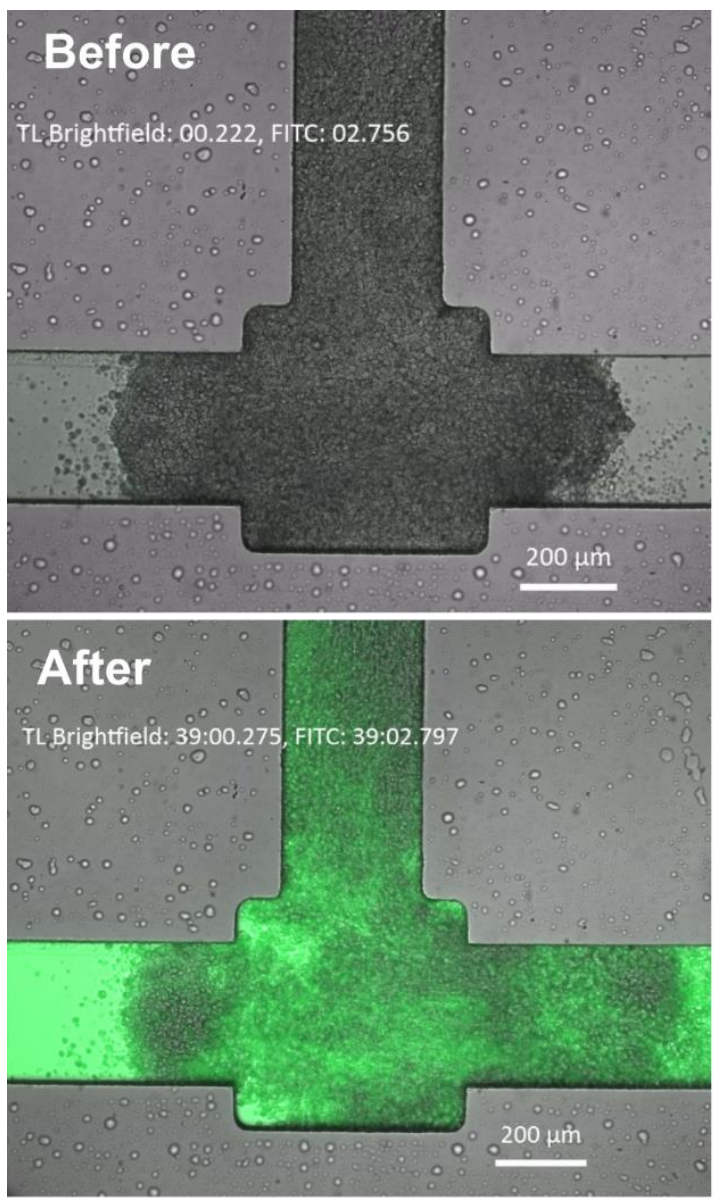

Loading rate 1
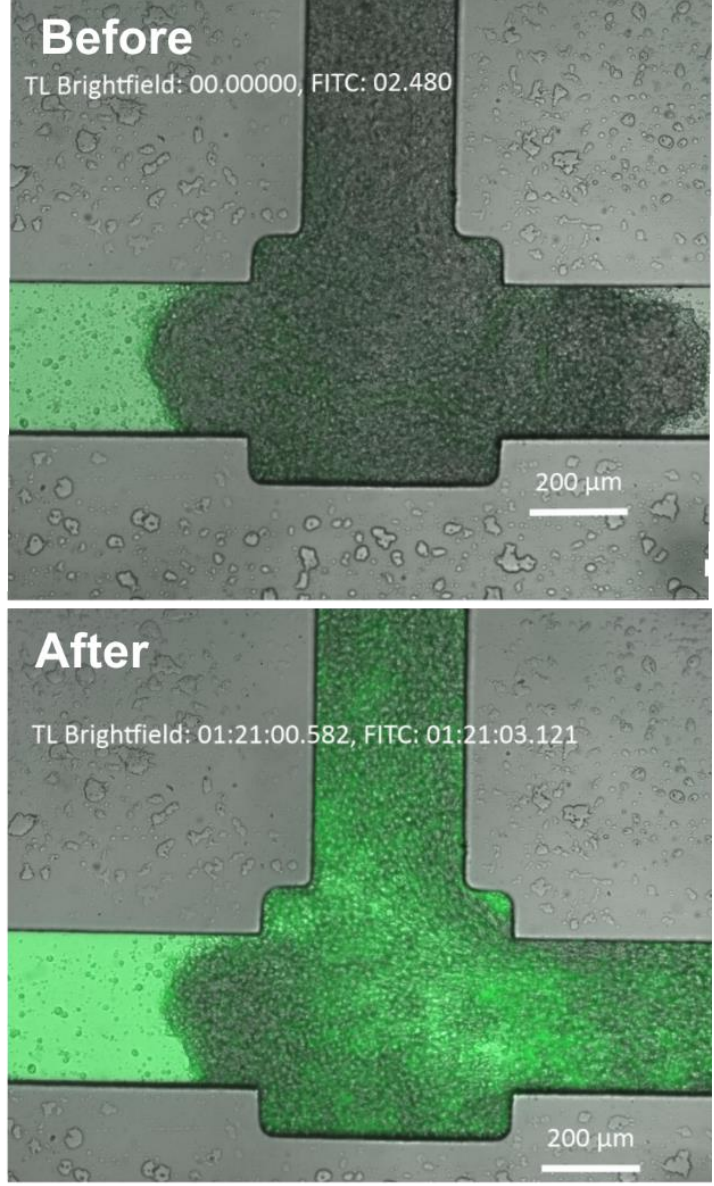

Loading rate 2

Figure 5.12 The deformation of cellular aggregates under two loading rates. Loading rate 1 at $1 \mu 1 \mathrm{~min}^{-2}$, loading rate 2 at $0.5 \mu 1 \mathrm{~min}^{-2}$. The cellular aggregate under loading rate 1 was more damaged than under loading rate 2 . The time scale for the flow to damage the fracture appearance under loading rate 1 was also shorter than loading rate 2 .

\subsubsection{Validation of theoretical poroelastic model for the first regime of cellular aggregate behavior under interstitial flow}

Our experiment results showed that the theoretical model developed in 5.1 is applicable in the first regime behavior of the cellular aggregate under interstitial flow, before the fracture happens.

The model used Kozeny-Carman equation to estimate the permeability of the cylindrical poroelastic model, with the assumptions that a laminar flow occurs through a 
parallel ensemble of tortuous tubes of similar strength and the porosity of the model is much smaller than 1 .

Comparing these hypotheses with our experiment, we first observe that the laminar flow assumption is certainly valid, since the experimental Reynolds number is very small (much smaller than 1). The hypothesis of flow occurring through parallel tubes of similar length is not valid after fracture occurs, because fracture creates a single preferential tube for the flow, but the assumption appears reasonable in the absence of fracture. The picture of a packed bed of spheres is a rough approximation to the geometry of the aggregate: in reality, cells as not quite spherical, but we expect nevertheless the physical scaling arguments that yield the Kozeny-Carman relationship to hold. The low porosity assumption is correct, as we expect porosity of the aggregate to be very low, especially for our highly adhesive cell line. In summary, the Kozeny-Carman equation appears a reasonable, simple model, as long as fracture does not occur, whereas it would need to be modified to describe the flow regime after aggregate fracture. 


\section{CHAPTER 6 Conclusion and perspectives}

This chapter summarizes the results that have been achieved throughout the project. The main points in two topics of CTC isolation and interstitial flow characterization are stated.

In the last part of this chapter, we propose some further improvements and new approaches for this project. 


\subsection{Conclusion}

\subsubsection{The flexibility of microfluidics on flow application in metastasis research}

In this project, we presented two designs of microfluidic platforms to investigate two major factors that influence on metastasis derivation.

In chapter 4, we have introduced a microfluidic device to sequentially isolate single cells which can be used to capture and separate single CTCs from other cell types in the blood stream. This device is also suitable in applications of particle isolation and size separation.

In chapter 5, we characterized interstitial flow through a model tumor. Our device allows applying different flow profiles to the model tumor, and capturing the pressure difference across the model tumor as well as live imaging of the tumor responses to the flow in real-time.

These results indicate the enormous potential of microfluidics in different experimental setups with different purposes of study.

\subsubsection{The sequential isolation of floating tumor cells under continuous flow}

We have developed an innovative sequential cell isolation device with up to $100 \%$ isolation yield and $>95 \%$ sequential isolation efficiency through a series of microsieves arranged in a novel arrangement. By applying a constant sample flow-rate through a microchannel, particles or cells are trapped automatically and sequentially in the microsieves at precisely defined positions. With low Reynolds number laminar flow used in the microfluidic channel, the structural integrity cell membrane, cell viability and genome integrity in the nucleus are guaranteed, as required for subsequent detection and 
analysis. Furthermore, the throughput of the system can be substantially increased with the parallelization of the microtrapper design, reducing the processing time to approximately 10 minutes. The reported framework of sequential cell isolation microstructures offers a robust platform for isolation of floating cells in biosamples, and also provides a fitting platform for the fundamental studies and clinical applications, such as the investigation of tumor drug resistance and tumor heterogeneity; and demonstration of on-chip single cell labelling and viability tests.

\subsubsection{The characterization of interstitial flow through a model tumor}

In this study, we have introduced a simple but effective microfluidic device to characterize flow through a tumor modelled by a cellular aggregate. We developed a novel method of transferring the spheroidal cellular aggregates from outside into the device by a typical syringe pump.

Our first microfluidic design was calibrated by applying flow through an inert porous medium. We have successfully achieved the expected linear relation between the flow and pressure difference across a chamber of collagen gel. We were able to quantify the collagen gel permeability in agreement with data from previous studies.

The results from our first design also demonstrated that the cellular aggregate behaved under the interstitial flow following two regimes: poroelasticity and solid plasticity, which are separated at a transient point, corresponding to fracture inside the cellular aggregate. During experiments with the first design, we have realized the limitations of the system, most notably that the pressure readings from the manometer were affected by the evaporation of the fluid when the flow velocity was $\sim 0.2 \mu \mathrm{m} \mathrm{s}^{-1}$. Additionally, the first design only allowed us to apply stepping flow rates to the cellular 
aggregate due to its delay time to reach each steady state. Nevertheless, these limitations did not deny our findings about the aggregate fracture.

In the second design, we have further characterized this transient point and found that the critical pressure at the transient point depended on the different loading rates of the flow. Specifically, the higher loading rate of the flow resulted in a higher critical pressure for aggregate fracture.

We also developed a simplified theoretical model for the cellular aggregate behaving as a poroelastic material. This model was able to represent the cellular aggregate behavior at the first regime following 'poroelasticity'. The characteristic curve between pressure difference and flow rate observed experimentally can thus be interpreted in terms of the ratio between the ability of the hydrostatic pressure to induce flow through aggregate pores and its ability to elastically deform the cellular aggregate.

\subsection{New perspectives}

\subsubsection{Enhancement of CTC isolation ability in inertia-based sorting devices by combination of the sequential isolation device}

As introduced in 2.1.1, inertia-based sorting devices [71, 108] have the advantage of fast sorting of CTCs from blood cells with efficiency of $\sim 85 \%$, but they lack of single cell isolation ability.

Our sequential cell isolation device has proven its ability in isolating single cells with high isolation efficiency (>95\%) and sorting particles in different sizes. Here, we propose an idea of combining our microfluidic device with inertia-based sorting devices. The collection of cells from the inertia-based sorting devices can be continued to isolate using our microfluidic design. This combination will fasten the whole isolation process and even increase the isolation efficiency. 


\subsubsection{Isolation of CTCs in practical applications}

Our sequential cell isolation device has shown the ability of separation of microbeads due to the size differences. We also proposed a strategy to capture the single CTCs from other blood cells such as red blood cells, white blood cells, and platelets. However, we still lack of practical experiments to confirm the feasibility of the device. Here, we present a detailed procedure for the next step of our experiment to separate the single CTCs from blood cells:

1. Dilute the liquid sample containing red blood cells, white blood cells, platelets and CTCs to the concentration at $10^{6}$ cell $\mathrm{ml}^{-1}$. This is to prevent the cells from not getting stuck at the injector of the device.

2. Inject the liquid sample to the device. The process will finish when all the liquid sample is run out. In case that all the microsieves are filled with cells, we can continue the process with an another device.

3. All the cells which have smaller size than the microsieve gap, such as red blood cells, platelets and some small white blood cells, will not be trapped. They will flow all to the outlet. The CTCs and some large size white blood cells will remain in the microsieves of the devices.

4. Differentiate the CTCs from the remaining white blood cells by on-chip fluorescence immuno- staining [71], fluorescence- activated cell sorting (FACS) [165], or immuno- magnetic separation [165], or density gradient centrifugation [166].

Additionally, the tumor cells detaching from their primary tumor can travel in aggregate form. In another case, the tumor cells can be covered by the platelets adhering to their surface $[23,24,26]$. These actions make the CTCs in real situation arbitrary in size and shape. Thus, we need to carry out an experiment where we produce a mixture of 
cellular aggregates in different sizes and shapes and use our microfluidic device to capture those aggregates. The optimization process and parametric tests for the cellular aggregates will be to be conducted.

\subsubsection{Improvement of the microfluidic design to characterize interstitial flow through the cellular aggregate with the ability of quantifying the aggregate permeability}

In 5.2.4, we have mentioned the limitations of the first design to characterize flow through a cellular aggregate. Although the problem with medium evaporation at $37^{\circ} \mathrm{C}$ has been resolved in the second design, the current design is still unable to quantify aggregate permeability. Indeed, in the second design, we were able to quantify the equivalent hydrodynamic resistance of the cell chamber and the side channel, but the resistance of the cell chamber could not be accurately measured due to lack of flow rate measurements that can determine how much of the flow goes through the cell chamber. The very low velocity of the flow through the cellular aggregate makes it difficult to be measured in a microscale device.

In order to overcome this difficulty, we propose an installation of a microflow meter at the cell chamber channel. The flow rate through the cellular aggregate will be measured and recorded by the flow meter in real-time. The microflow meter is required to have the ability in measuring flow rates that approximately lower than $1 \mu 1 \mathrm{~min}^{-1}$ in high precision with the real-time data logging to a computer.

After collecting the data of flow rate going through the cellular aggregate, we can calculate the hydrodynamic resistance of the cellular aggregate chamber. We can quantify how this resistance changes during applying the flow. This result will allow us to quantify aggregate permeability and to interpret cell aggregate behavior in terms of the poroelastic theoretical model presented in this thesis. 


\subsubsection{Characterizing flow through a cellular aggregate with an extracellular matrix}

The study of flow through a cellular aggregate modeling a cancer tumor can be further developed with the appearance of extracellular matrix (see Figure 6.1).

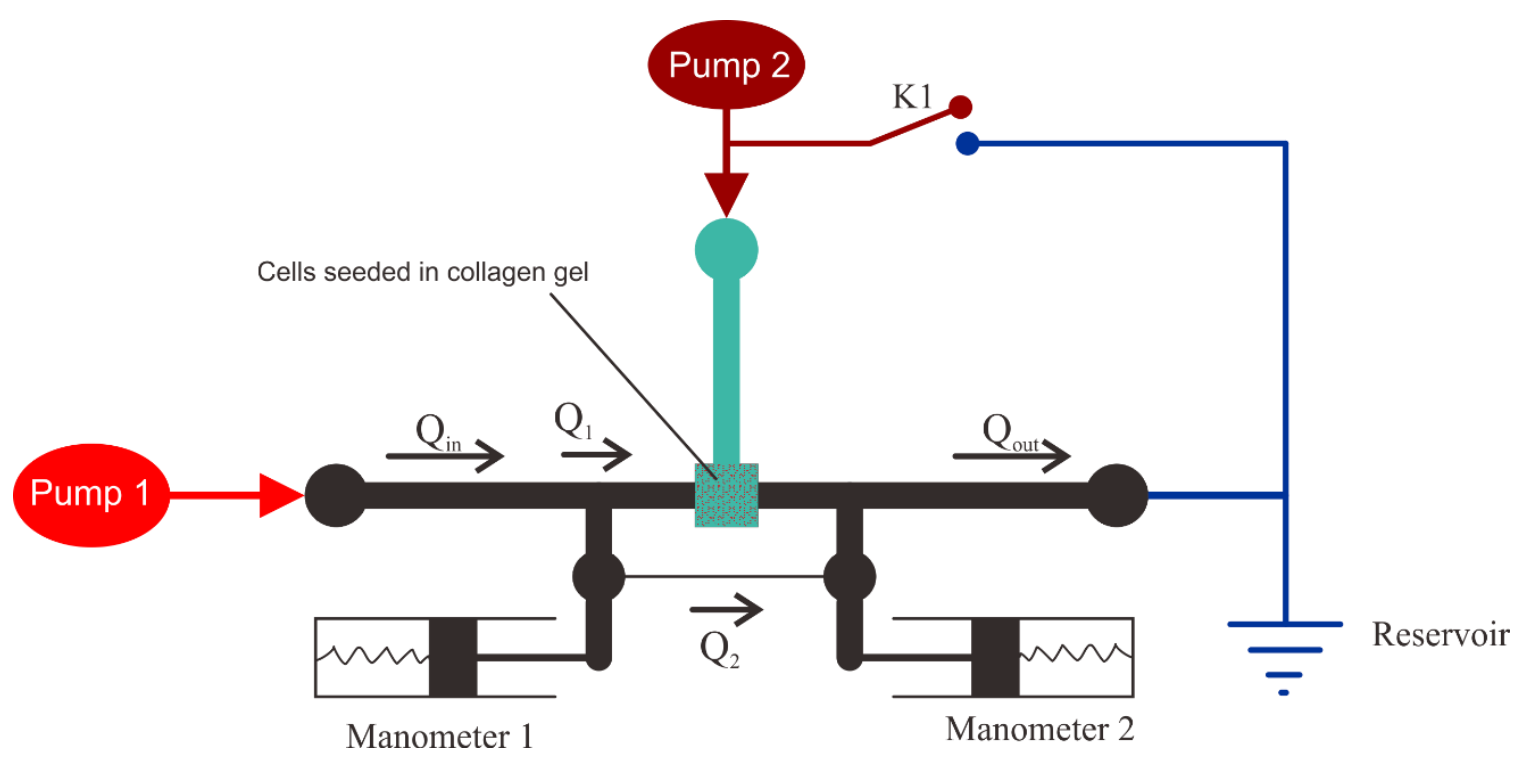

Figure 6.1 Proposed design to characterize flow through a cellular aggregate (red chamber) placed between two chambers of collagen gel (blue chamber).

Here we present a new approach for interstitial flow characterization. In real tumor environment, tumor mass is surrounded by the extracellular matrix. This matrix acts like a shield to reduce the shear effects of flow on the tumor cells [82]. We propose a new design for the flow chamber, which includes cells seeded in collagen gel. The collagen gel with cells will also be introduced to the chamber and then different flow profiles will be applied through the chamber.

The results of pressure difference and flow rate across the chambers of collagen gel will be compared with our previous studies. 


\subsubsection{Developing theoretical model for two regimes of cellular aggregate behaviors}

The poroelastic model does not describe the occurrence of aggregate fracture. To theoretically model aggregate fracture, we plan to develop an extension of the model of Gonzalez-Rodriguez et al. [138]. In this theoretical model, the rheological behavior of a cellular aggregate leading to fracture is described by analogy with fracture in inert polymer glues. In the planned model, we will use this rheological description and investigate its fracture response when hydrodynamic forces are acted upon the cellular aggregate.

This model will help us predict the aggregate fracture happening and contribute to our understanding on the onset of metastasis.

\subsubsection{Investigation on the interdependence of interstitial flow effects and biochemical environment changes to a tumor}

Metastasis is a complex process, which is influenced by multiple effects from both physical and bio-chemical factors. This thesis has investigated the physical impact of interstitial flow on tumor mass, however, there is still lack of understanding about how the bio-chemical environment changes to the cell-cell adhesion of the tumor. Since cancer cells are less adhesive than normal cells, and the cell-cell adhesion becomes weaker over time [9], the tumor cells will have the potential to detach from their primary tumor, which leads to metastasis. Moreover, this cell-cell adhesion is also affected by the cell-substrate interactions $[136,137]$. Thus, it is important to investigate the interdependence of both physical effects from interstitial flow and cell-cell adhesion changes in tumor due to biochemical substrate changes, which can provide a good prediction tool for metastasis diagnosis. 
We propose an idea of using our microfluidic devices to characterize different flow conditions to a series of cellular aggregates which possess different levels of E-cadherins representing different levels of cell-cell adhesion. Moreover, we also study the effects of variation of cell-substrate interactions on cell-cell adhesion under the flow. By coating our microchannels with different chemical treatments, either fibronectin (high cell-substrate adhesion) or PEG (low adhesion), experiments by applying flow through cellular aggregates in different cell-substrate adhesion levels will be conducted. A good synthesis of flow effects with different levels of E-cadherins in the cellular aggregates will result in a deeper insight for metastasis diagnosis.

\subsubsection{Integrated microfluidic device to quantify cancer cell detachment from a primary tumor}

We are interested in quantifying cell detachment from the primary tumor induced by interstitial flow. To address this topic, we plan to integrate the two microfluidic devices developed in this thesis into a design to quantify single cell detachment from a tumor (Figure 6.2).

A flow will be applied through the cellular aggregate. The single cells escaped from the tumor will be captured in the isolation channel. The experiment will be stopped when the single cells fill all the micro-sieves inside the isolation channel. The cells captured in the isolation channel will go through live/dead test to further quantify the viability of the cells after the detaching from the tumor.

The advantage of this design is that we can let the whole run proceed automatically and compare different flow settings in the same time scale of the experiment. For example, we can quantify how many cells escaped from the tumor in 1 day under 5 loading rates of the flow. The main goal of this experiment is to quantify how 
cell detachment from the model tumor depends on the characteristics of the flow profile applied to it.

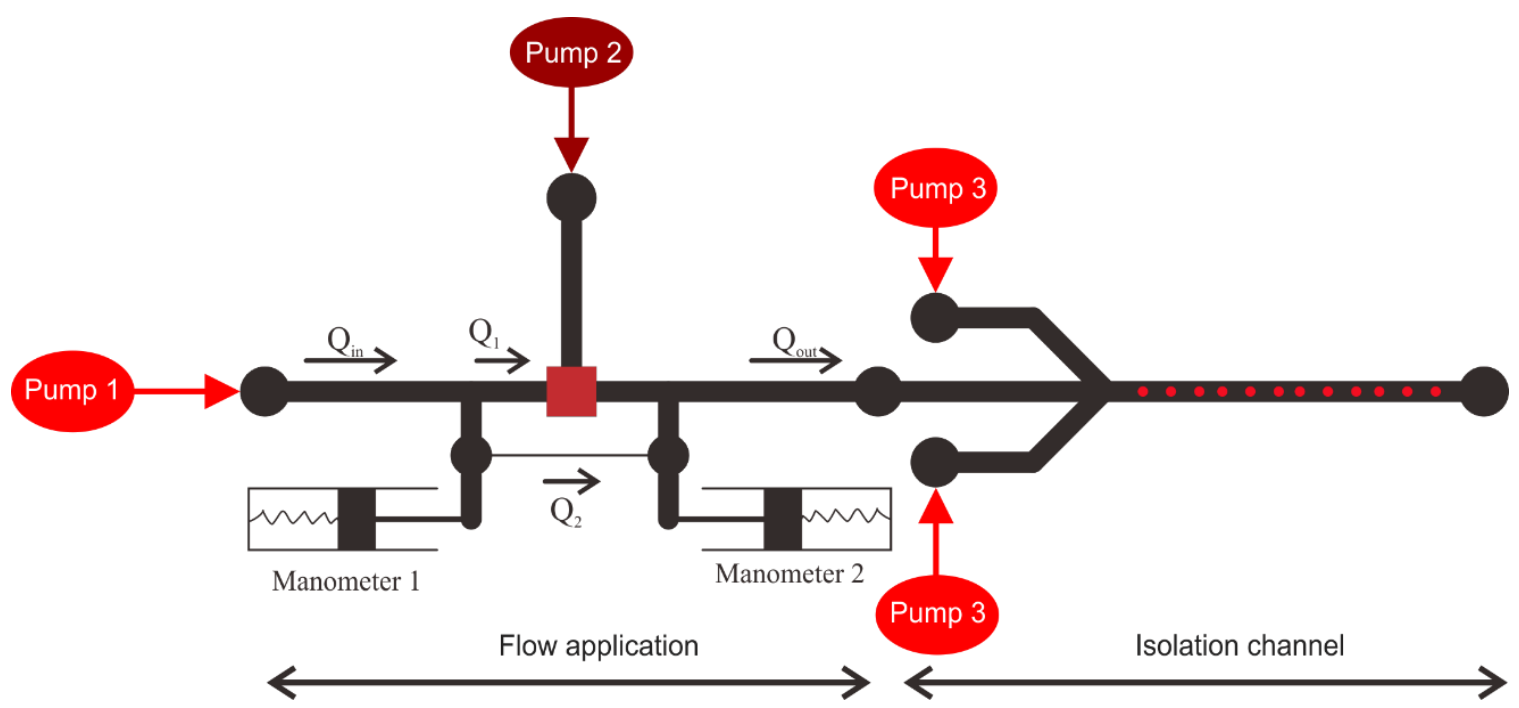

Figure 6.2 Proposal of an integrated device including the microfluidic design to generate interstitial flow through a cellular aggregate associated with extracellular matrix, connected to an isolation channel to capture the single cells escaped from the tumor.

\subsubsection{Overall vision and further approaches of the project}

The thesis introduced different uses of microfluidics to assist in vitro research on metastasis which is the main cause of death among millions of cancer patients. Taking the full advantages of microfluidics, we can develop many devices and applications in contribution to metastasis diagnosis process with robust techniques, high efficiency, and low cost.

Further tests on in vivo samples or conducting more realistic experiments will be the important approaches for the project. The combination between our current sequential cell isolation device [56] with inertia-based cell sorting devices [71, 108] has the potential to provide a fast and high efficient solution to differentiate CTCs from other cell types in a blood sample. Additionally, a further developed study about the effects of interstitial flow on cellular aggregates with different cell-cell adhesion can contribute more understanding on metastasis. For example, a theoretical model to characterize the fracture 
happened at a cellular aggregate under interstitial flow, which can be extended from Gonzalez-Rodriguez et al. [138], will help a robust model for metastasis prediction. Succession on these assays will open a new practice for metastasis research that is closer to clinical applications.

In addition to metastasis research, microfluidics is widely used in other biophysical assays. For instance, microfluidics provides a robust and high precision tool for malaria detection [183], helps study the chemotaxis of bacteria [145]. Utilizing the benefits of microfluidics to assist other biophysical aspects will also be a future approach for the candidate. 


\section{References}

[1] B. W. Stewart and C. Wild, "World Cancer Report 2014. International Agency for Research on Cancer," World Health Organization, vol. 505, 2014.

[2] G. Karp, Cell and molecular biology: concepts and experiments, 3rd ed.: John Wiley \& Sons, Inc., 2002.

[3] J. Cairns, "Mutation selection and the natural history of cancer," Nature, vol. 255, pp. 197-200, 1975.

[4] L. H. Hartwell and M. B. Kastan, "Cell cycle control and cancer," Science, vol. 266, p. 1821, 1994.

[5] R. Holliday and J. E. Pugh, "DNA modification mechanisms and gene activity during development," Cold Spring Harbor Monograph Archive, vol. 32, pp. 639645, 1996.

[6] I. P. Tomlinson, M. Novelli, and W. Bodmer, "The mutation rate and cancer," Proceedings of the National Academy of Sciences, vol. 93, pp. 14800-14803, 1996.

[7] J. S. Haldane, "The rate of mutation of human genes," Hereditas, vol. 35, pp. 267273, 1949.

[8] D. Wirtz, K. Konstantopoulos, and P. C. Searson, "The physics of cancer: the role of physical interactions and mechanical forces in metastasis," Nat Rev Cancer, vol. 11, pp. 512-522, 07//print 2011. Available: http://dx.doi.org/10.1038/nrc3080

[9] H. Oka, H. Shiozaki, K. Kobayashi, M. Inoue, H. Tahara, T. Kobayashi, et al., "Expression of E-cadherin cell adhesion molecules in human breast cancer tissues and its relationship to metastasis," Cancer research, vol. 53, pp. 1696-1701, 1993.

[10] L. Hayflick, "The illusion of cell immortality," British journal of cancer, vol. 83, p. 841, 2000.

[11] C. B. Harley, N. Kim, K. Prowse, S. Weinrich, K. Hirsch, M. West, et al., "Telomerase, cell immortality, and cancer," in Cold Spring Harbor symposia on quantitative biology, 1994, pp. 307-315.

[12] F. Macdonald, C. Ford, and A. Casson, Molecular biology of cancer: Taylor \& Francis, 2004.

[13] G. Poste and I. J. Fidler, "The pathogenesis of cancer metastasis," Nature, vol. 283, pp. 139-146, 1980.

[14] C. A. Klein, "The metastasis cascade," Science, vol. 321, pp. 1785-1787, 2008.

[15] J. Sleeman and P. S. Steeg, "Cancer metastasis as a therapeutic target," European Journal of Cancer, vol. 46, pp. 1177-1180, May 2010.

[16] L. Liotta and W. Stetler-Stevenson, "Metalloproteinases and cancer invasion," in Seminars in cancer biology, 1990, pp. 99-106.

[17] I. J. Fidler, D. M. Gersten, and I. R. Hart, "The biology of cancer invasion and metastasis," Advances in cancer research, vol. 28, pp. 149-250, 1978.

[18] J. Folkman, "Role of angiogenesis in tumor growth and metastasis," in Seminars in oncology, 2002, pp. 15-18.

[19] D. W. Siemann, "Vascular Targeting Agents," Horizons in Cancer Therapeutics: From Bench to Bedside, vol. 3, pp. 4-15, 2002.

[20] T. Tsuji, S. Ibaragi, and G.-f. Hu, "Epithelial-mesenchymal transition and cell cooperativity in metastasis," Cancer research, vol. 69, pp. 7135-7139, 2009.

[21] F. van Zijl, G. Krupitza, and W. Mikulits, "Initial steps of metastasis: cell invasion and endothelial transmigration," Mutation Research/Reviews in Mutation Research, vol. 728, pp. 23-34, 2011. 
[22] I. J. Fidler, "Metastasis: quantitative analysis of distribution and fate of tumor emboli labeled with 125I-5-iodo-2'-deoxyuridine," Journal of the National Cancer Institute, vol. 45, pp. 773-782, 1970.

[23] A. Boucharaba, C.-M. Serre, S. Grès, J. S. Saulnier-Blache, J.-C. Bordet, J. Guglielmi, et al., "Platelet-derived lysophosphatidic acid supports the progression of osteolytic bone metastases in breast cancer," Journal of Clinical Investigation, vol. 114, pp. 1714-1725, 2004.

[24] G. J. Gasic, T. B. Gasic, N. Galanti, T. Johnson, and S. Murphy, "Platelettumor-cell interactions in mice. The role of platelets in the spread of malignant disease," International Journal of Cancer, vol. 11, pp. 704-718, 1973.

[25] M. Labelle, S. Begum, and R. O. Hynes, "Direct signaling between platelets and cancer cells induces an epithelial-mesenchymal-like transition and promotes metastasis," Cancer cell, vol. 20, pp. 576-590, 2011.

[26] K. V. Honn, D. G. Tang, and J. D. Crissman, "Platelets and cancer metastasis: a causal relationship?," Cancer and Metastasis Reviews, vol. 11, pp. 325-351, 1992.

[27] American Society for Biochemistry and Molecular Biology. (2007, January 18, 2014). How Cancer Spreads By Aggregating Platelets. [Web]. Available: http://www.sciencedaily.com/releases/2007/08/070830150049.htm

[28] E. B. Voura, J. K. Jaiswal, H. Mattoussi, and S. M. Simon, "Tracking metastatic tumor cell extravasation with quantum dot nanocrystals and fluorescence emission-scanning microscopy," Nature medicine, vol. 10, pp. 993-998, 2004.

[29] S. Weis, J. Cui, L. Barnes, and D. Cheresh, "Endothelial barrier disruption by VEGF-mediated Src activity potentiates tumor cell extravasation and metastasis," The Journal of cell biology, vol. 167, pp. 223-229, 2004.

[30] P. Vaupel, F. Kallinowski, and P. Okunieff, "Blood flow, oxygen and nutrient supply, and metabolic microenvironment of human tumors: a review," Cancer research, vol. 49, pp. 6449-6465, 1989.

[31] G. L. Nicolson, "Cancer metastasis: tumor cell and host organ properties important in metastasis to specific secondary sites," Biochimica et Biophysica Acta (BBA)-Reviews on Cancer, vol. 948, pp. 175-224, 1988.

[32] "Global Cancer Facts \& Figures 3rd Edition," Atlanta: American Cancer Society, 2015.

[33] Harvard Health Publication, "Breast Cancer," ed: www.drugs.com.

[34] B. R. Lester and J. B. McCarthy, "Tumor cell adhesion to the extracellular matrix and signal transduction mechanisms implicated in tumor cell motility, invasion and metastasis," Cancer and Metastasis Reviews, vol. 11, pp. 31-44, 1992.

[35] K. K. Wary, "Recognizing scientific excellence in the biology of cell adhesion," Cell Commun Signal, vol. 3, 2005.

[36] M. Guarino, B. Rubino, and G. Ballabio, "The role of epithelial-mesenchymal transition in cancer pathology," Pathology, 2009.

[37] H. Ninomiya and R. Winklbauer, "Epithelial coating controls mesenchymal shape change through tissue-positioning effects and reduction of surface-minimizing tension," Nat Cell Biol, vol. 10, pp. 61-69, 01//print 2008. Available: http://dx.doi.org/10.1038/ncb1669

[38] W. J. Polacheck, I. K. Zervantonakis, and R. D. Kamm, "Tumor cell migration in complex microenvironments," Cellular and Molecular Life Sciences, pp. 1-22, 2012.

[39] W. J. Polacheck, R. Li, S. G. Uzel, and R. D. Kamm, "Microfluidic platforms for mechanobiology," Lab on a Chip, vol. 13, pp. 2252-2267, 2013. 
[40] Y. Shin, S. Han, J. S. Jeon, K. Yamamoto, I. K. Zervantonakis, R. Sudo, et al., "Microfluidic assay for simultaneous culture of multiple cell types on surfaces or within hydrogels," Nature protocols, vol. 7, pp. 1247-1259, 2012. Available: http://dx.doi.org/10.1038/nprot.2012.051

[41] A. Boussommier-Calleja, R. Li, M. B. Chen, S. C. Wong, and R. D. Kamm, "Microfluidics: A New Tool for Modeling Cancer-Immune Interactions," Trends in Cancer, vol. 2, pp. 6-19, 1// 2016. Available:

http://www.sciencedirect.com/science/article/pii/S2405803315000837

[42] S. G. Uzel, A. Pavesi, and R. D. Kamm, "Microfabrication and microfluidics for muscle tissue models," Progress in biophysics and molecular biology, vol. 115, pp. 279-293, 2014.

[43] S. Chung, R. Sudo, P. J. Mack, C.-R. Wan, V. Vickerman, and R. D. Kamm, "Cell migration into scaffolds under co-culture conditions in a microfluidic platform," Lab on a Chip, vol. 9, pp. 269-275, 2009.

[44] W. J. Polacheck, J. L. Charest, and R. D. Kamm, "Interstitial flow influences direction of tumor cell migration through competing mechanisms," Proceedings of the National Academy of Sciences, vol. 108, pp. 11115-11120, 2011. Available: http://dx.doi.org/10.1073/pnas.1103581108

[45] U. Haessler, J. C. M. Teo, D. Foretay, P. Renaud, and M. A. Swartz, "Migration dynamics of breast cancer cells in a tunable 3D interstitial flow chamber," Integrative Biology, vol. 4, pp. 401-409, 2012. Available: http://dx.doi.org/10.1039/c1ib00128k

[46] I. K. Zervantonakis, S. K. Hughes-Alford, J. L. Charest, J. S. Condeelis, F. B. Gertler, and R. D. Kamm, "Three-dimensional microfluidic model for tumor cell intravasation and endothelial barrier function," Proceedings of the National Academy of Sciences, vol. 109, pp. 13515-13520, 2012.

[47] M. K. Shin, S. K. Kim, and H. Jung, "Integration of intra-and extravasation in one cell-based microfluidic chip for the study of cancer metastasis," Lab on a chip, vol. 11, pp. 3880-3887, 2011.

[48] H. Lee, W. Park, H. Ryu, and N. L. Jeon, "A microfluidic platform for quantitative analysis of cancer angiogenesis and intravasationa),"

Biomicrofluidics, vol. 8, p. 054102, 2014.

[49] J. S. Jeon, I. K. Zervantonakis, S. Chung, R. D. Kamm, and J. L. Charest, "In vitro model of tumor cell extravasation," PloS one, vol. 8, p. e56910, 2013.

[50] M. B. Chen, J. A. Whisler, J. S. Jeon, and R. D. Kamm, "Mechanisms of tumor cell extravasation in an in vitro microvascular network platform," Integrative Biology, vol. 5, pp. 1262-1271, 2013.

[51] J. S. Jeon, S. Bersini, M. Gilardi, G. Dubini, J. L. Charest, M. Moretti, et al., "Human 3D vascularized organotypic microfluidic assays to study breast cancer cell extravasation," Proceedings of the National Academy of Sciences, vol. 112, pp. 214-219, 2015.

[52] J. Kalchman, S. Fujioka, S. Chung, Y. Kikkawa, T. Mitaka, R. D. Kamm, et al., "A three-dimensional microfluidic tumor cell migration assay to screen the effect of anti-migratory drugs and interstitial flow," Microfluidics and Nanofluidics, pp. 1-13, 2012. Available: http://dx.doi.org/10.1007/s10404-012-1104-6

[53] C. Kim, J. Kasuya, J. Jeon, S. Chung, and R. D. Kamm, "A quantitative microfluidic angiogenesis screen for studying anti-angiogenic therapeutic drugs," Lab on a chip, vol. 15, pp. 301-310, 2015.

[54] G. M. Whitesides, "The origins and the future of microfluidics," Nature, vol. 442, pp. 368-373, 2006. 
[55] Z. Li, S. Y. Mak, A. Sauret, and H. C. Shum, "Syringe-pump-induced fluctuation in all-aqueous microfluidic system implications for flow rate accuracy," Lab on a Chip, vol. 14, pp. 744-749, 2014.

[56] Q. D. Tran, T. F. Kong, D. Hu, Marcos, and R. H. Lam, "Deterministic sequential isolation of floating cancer cells under continuous flow," Lab on a Chip, 2016. Available: http://dx.doi.org/10.1039/C6LC00615A

[57] V. Vickerman, J. Blundo, S. Chung, and R. Kamm, "Design, fabrication and implementation of a novel multi-parameter control microfluidic platform for three-dimensional cell culture and real-time imaging," Lab on a Chip, vol. 8, pp. 1468-1477, 2008. Available: http://dx.doi.org/10.1039/B802395F

[58] R. Sudo, S. Chung, I. K. Zervantonakis, V. Vickerman, Y. Toshimitsu, L. G. Griffith, et al., "Transport-mediated angiogenesis in 3D epithelial coculture," The FASEB Journal, vol. 23, pp. 2155-2164, July 1, 2009 2009. Available: http://www.fasebj.org/content/23/7/2155.abstract

[59] Z. Xu, Y. Gao, Y. Hao, E. Li, Y. Wang, J. Zhang, et al., "Application of a microfluidic chip-based 3D co-culture to test drug sensitivity for individualized treatment of lung cancer," Biomaterials, vol. 34, pp. 4109-4117, 2013.

[60] A. R. Aref, R. Y.-J. Huang, W. Yu, K.-N. Chua, W. Sun, T.-Y. Tu, et al., "Screening therapeutic EMT blocking agents in a three-dimensional microenvironment," Integrative Biology, vol. 5, pp. 381-389, 2013.

[61] Y. L. Huang, C.-k. Tung, A. Zheng, B. J. Kim, and M. Wu, "Interstitial flows promote amoeboid over mesenchymal motility of breast cancer cells revealed by a three dimensional microfluidic model," Integrative Biology, vol. 7, pp. 1402-1411, 2015. Available: http://dx.doi.org/10.1039/C5IB00115C

[62] H. Lu, L. Y. Koo, W. M. Wang, D. A. Lauffenburger, L. G. Griffith, and K. F. Jensen, "Microfluidic shear devices for quantitative analysis of cell adhesion," Analytical chemistry, vol. 76, pp. 5257-5264, 2004.

[63] S.-Y. Cheng, S. Heilman, M. Wasserman, S. Archer, M. L. Shuler, and M. Wu, "A hydrogel-based microfluidic device for the studies of directed cell migration," Lab on a Chip, vol. 7, pp. 763-769, 2007.

[64] E. Racila, D. Euhus, A. J. Weiss, C. Rao, J. McConnell, L. W. M. M. Terstappen, et al., "Detection and characterization of carcinoma cells in the blood,"

Proceedings of the National Academy of Sciences, vol. 95, pp. 4589-4594, April 14, 1998 1998. Available: http://www.pnas.org/content/95/8/4589.abstract

[65] K. Pantel, R. H. Brakenhoff, and B. Brandt, "Detection, clinical relevance and specific biological properties of disseminating tumour cells," Nat Rev Cancer, vol. 8, pp. 329-340, 05//print 2008. Available: http://dx.doi.org/10.1038/nrc2375

[66] Vortex Biosciences. Cancer \& CTCs. Available: http://vortexbiosciences.com/technology/

[67] G. P. Gupta and J. Massagué, "Cancer metastasis: building a framework," Cell, vol. 127, pp. 679-695, 2006.

[68] P. Paterlini-Brechot and N. L. Benali, "Circulating tumor cells (CTC) detection: clinical impact and future directions," Cancer letters, vol. 253, pp. 180-204, 2007.

[69] M. Ilie, V. Hofman, E. Long, O. Bordone, E. Selva, K. Washetine, et al., "Current challenges for detection of circulating tumor cells and cell-free circulating nucleic acids, and their characterization in non-small cell lung carcinoma patients. What is the best blood substrate for personalized medicine?," Annals of translational medicine, vol. 2, 2014. 
[70] Y. Chen, P. Li, P.-H. Huang, Y. Xie, J. D. Mai, L. Wang, et al., "Rare cell isolation and analysis in microfluidics," Lab on a Chip, vol. 14, pp. 626-645, 2014.

[71] H. W. Hou, M. E. Warkiani, B. L. Khoo, Z. R. Li, R. A. Soo, D. S.-W. Tan, et al., "Isolation and retrieval of circulating tumor cells using centrifugal forces,"

Scientific Reports, vol. 3, p. 1259, 2013. Available:

http://dx.doi.org/10.1038/srep01259

[72] R. H. Lam, "Trends of Automation of Integrated Microfluidics," Advances in Robotics \& Automation, vol. 3, p. 1000e119, 2013.

[73] P. Ertl, C. A. Emrich, P. Singhal, and R. A. Mathies, "Capillary Electrophoresis Chips with a Sheath-Flow Supported Electrochemical Detection System," Analytical Chemistry, vol. 76, pp. 3749-3755, 2004/07/01 2004. Available: http://dx.doi.org/10.1021/ac035282a

[74] C.-L. E. Helm, M. E. Fleury, A. H. Zisch, F. Boschetti, and M. A. Swartz, "Synergy between interstitial flow and VEGF directs capillary morphogenesis in vitro through a gradient amplification mechanism," Proceedings of the National Academy of Sciences of the United States of America, vol. 102, pp. 15779-15784, 2005.

[75] M. E. Fleury, K. C. Boardman, and M. A. Swartz, "Autologous morphogen gradients by subtle interstitial flow and matrix interactions," Biophysical journal, vol. 91, pp. 113-121, 2006.

[76] J. D. Shields, M. E. Fleury, C. Yong, A. A. Tomei, G. J. Randolph, and M. A. Swartz, "Autologous chemotaxis as a mechanism of tumor cell homing to lymphatics via interstitial flow and autocrine CCR7 signaling," Cancer cell, vol. 11, pp. 526-538, 2007. Available: http://dx.doi.org/10.1016/j.ccr.2007.04.020

[77] J. A. Pedersen, F. Boschetti, and M. A. Swartz, "Effects of extracellular fiber architecture on cell membrane shear stress in a 3D fibrous matrix," Journal of biomechanics, vol. 40, pp. 1484-1492, 2007.

[78] J. A. Pedersen, S. Lichter, and M. A. Swartz, "Cells in 3D matrices under interstitial flow: effects of extracellular matrix alignment on cell shear stress and drag forces," Journal of biomechanics, vol. 43, pp. 900-905, 2010.

[79] C. A. Kunder, A. L. St John, G. Li, K. W. Leong, B. Berwin, H. F. Staats, et al., "Mast cell-derived particles deliver peripheral signals to remote lymph nodes," Science Signaling, vol. 206, p. 2455, 2009.

[80] M. A. Swartz, "The physiology of the lymphatic system," Advanced drug delivery reviews, vol. 50, pp. 3-20, 2001.

[81] G. W. Schmid-Schonbein, "Microlymphatics and lymph flow," Physiological reviews, vol. 70, pp. 987-1028, 1990.

[82] A. C. Shieh and M. A. Swartz, "Regulation of tumor invasion by interstitial fluid flow," Physical Biology, vol. 8, p. 015012, 2011. Available:

http://stacks.iop.org/1478-3975/8/i=1/a=015012

[83] T. P. Padera, A. H. Kuo, T. Hoshida, S. Liao, J. Lobo, K. R. Kozak, et al., "Differential response of primary tumor versus lymphatic metastasis to VEGFR-2 and VEGFR-3 kinase inhibitors cediranib and vandetanib," Molecular cancer therapeutics, vol. 7, pp. 2272-2279, 2008.

[84] M. Dadiani, V. Kalchenko, A. Yosepovich, R. Margalit, Y. Hassid, H. Degani, et al., "Real-time imaging of lymphogenic metastasis in orthotopic human breast cancer," Cancer research, vol. 66, pp. 8037-8041, 2006.

[85] J. Sleeman, A. Schmid, and W. Thiele, "Tumor lymphatics," Semin Cancer Biol, vol. 19, pp. 285-97, Oct 2009. 
[86] A. Ruddell, M. I. Harrell, S. Minoshima, K. R. Maravilla, B. M. Iritani, S. W. White, et al., "Dynamic contrast-enhanced magnetic resonance imaging of tumorinduced lymph flow," Neoplasia (New York, NY), vol. 10, p. 706, 2008.

[87] M. I. Harrell, B. M. Iritani, and A. Ruddell, "Tumor-induced sentinel lymph node lymphangiogenesis and increased lymph flow precede melanoma metastasis," The American journal of pathology, vol. 170, pp. 774-786, 2007.

[88] S. R. Chary and R. K. Jain, "Direct measurement of interstitial convection and diffusion of albumin in normal and neoplastic tissues by fluorescence photobleaching," Proceedings of the National Academy of Sciences of the United States of America, vol. 86, pp. 5385-5389, Jul 1989. Available: http://dx.doi.org/10.1073/pnas.86.14.5385

[89] H. Dafni, T. Israely, Z. M. Bhujwalla, L. E. Benjamin, and M. Neeman, "Overexpression of Vascular Endothelial Growth Factor 165 Drives Peritumor Interstitial Convection and Induces Lymphatic Drain Magnetic Resonance Imaging, Confocal Microscopy, and Histological Tracking of Triple-labeled Albumin," Cancer research, vol. 62, pp. 6731-6739, 2002.

[90] T. P. Butler, F. H. Grantham, and P. M. Gullino, "Bulk transfer of fluid in the interstitial compartment of mammary tumors," Cancer research, vol. 35, pp. 3084-3088, 1975.

[91] R. K. Jain and L. T. Baxter, "Mechanisms of heterogeneous distribution of monoclonal-antibodies and other macromolecules in tumors - significance of elevated interstitial pressure," Cancer Research, vol. 48, pp. 7022-7032, Dec 1988. Available: http://cancerres.aacrjournals.org/content/48/24_Part_1/7022

[92] Y. Boucher and R. K. Jain, "Microvascular pressure is the principal driving force for interstitial hypertension in solid tumors: implications for vascular collapse," Cancer research, vol. 52, pp. 5110-5114, 1992.

[93] J. R. Less, M. C. Posner, Y. Boucher, D. Borochovitz, N. Wolmark, and R. K. Jain, "Interstitial hypertension in human breast and colorectal tumors," Cancer research, vol. 52, pp. 6371-6374, 1992.

[94] Y. Boucher, M. Leunig, and R. K. Jain, "Tumor angiogenesis and interstitial hypertension," Cancer research, vol. 56, pp. 4264-4266, 1996.

[95] M. A. Swartz and A. W. Lund, "Lymphatic and interstitial flow in the tumour microenvironment: linking mechanobiology with immunity," Nature Reviews Cancer, vol. 12, pp. 210-219, 2012. Available: http://dx.doi.org/10.1038/nrc3186

[96] M. A. Swartz and M. E. Fleury, "Interstitial flow and its effects in soft tissues," Annu. Rev. Biomed. Eng., vol. 9, pp. 229-256, 2007.

[97] E. K. Sackmann, A. L. Fulton, and D. J. Beebe, "The present and future role of microfluidics in biomedical research," Nature, vol. 507, pp. 181-189, 2014.

[98] J. P. Smith, A. C. Barbati, S. M. Santana, J. P. Gleghorn, and B. J. Kirby, "Microfluidic transport in microdevices for rare cell capture," Electrophoresis, vol. 33, pp. 3133-3142, 2012.

[99] P. Sajeesh and A. K. Sen, "Particle separation and sorting in microfluidic devices: a review," Microfluidics and nanofluidics, vol. 17, pp. 1-52, 2014.

[100] J.-C. Baret, O. J. Miller, V. Taly, M. Ryckelynck, A. El-Harrak, L. Frenz, et al., "Fluorescence-activated droplet sorting (FADS): efficient microfluidic cell sorting based on enzymatic activity," Lab on a Chip, vol. 9, pp. 1850-1858, 2009.

[101] K. E. McCloskey, L. R. Moore, M. Hoyos, A. Rodriguez, J. J. Chalmers, and M. Zborowski, "Magnetophoretic cell sorting is a function of antibody binding capacity," Biotechnology progress, vol. 19, pp. 899-907, 2003. 
[102] D. R. Gossett, W. M. Weaver, A. J. Mach, S. C. Hur, H. T. K. Tse, W. Lee, et al., "Label-free cell separation and sorting in microfluidic systems," Analytical and bioanalytical chemistry, vol. 397, pp. 3249-3267, 2010.

[103] P. Chen, X. Feng, R. Hu, J. Sun, W. Du, and B.-F. Liu, "Hydrodynamic gating valve for microfluidic fluorescence-activated cell sorting," Analytica chimica acta, vol. 663, pp. 1-6, 2010.

[104] X. Wang, S. Chen, M. Kong, Z. Wang, K. D. Costa, R. A. Li, et al., "Enhanced cell sorting and manipulation with combined optical tweezer and microfluidic chip technologies," Lab on a Chip, vol. 11, pp. 3656-3662, 2011.

[105] T. F. Kong, W. Ye, W. K. Peng, H. W. Hou, P. R. Preiser, N.-T. Nguyen, et al., "Enhancing malaria diagnosis through microfluidic cell enrichment and magnetic resonance relaxometry detection," Scientific reports, vol. 5, 2015.

[106] G. Mernier, N. Piacentini, T. Braschler, N. Demierre, and P. Renaud, "Continuous-flow electrical lysis device with integrated control by dielectrophoretic cell sorting," Lab on a Chip, vol. 10, pp. 2077-2082, 2010. Available: http://dx.doi.org/10.1039/C000977F

[107] M. Chabert and J.-L. Viovy, "Microfluidic high-throughput encapsulation and hydrodynamic self-sorting of single cells," Proceedings of the National Academy of Sciences, vol. 105, pp. 3191-3196, 2008.

[108] M. Dhar, J. Wong, A. Karimi, J. Che, C. Renier, M. Matsumoto, et al., "High efficiency vortex trapping of circulating tumor cells," Biomicrofluidics, vol. 9, p. 064116, 2015. Available: http://dx.doi.org/10.1063/1.4937895

[109] Y. Tang, J. Shi, S. Li, L. Wang, Y. E. Cayre, and Y. Chen, "Microfluidic device with integrated microfilter of conical-shaped holes for high efficiency and high purity capture of circulating tumor cells," Scientific Reports, vol. 4, 2014. Available: http://dx.doi.org/10.1038/srep06052

[110] W. Chen, N. T. Huang, B. Oh, R. H. Lam, R. Fan, T. T. Cornell, et al., "Surface-Micromachined Microfiltration Membranes for Efficient Isolation and Functional Immunophenotyping of Subpopulations of Immune Cells," Advanced healthcare materials, vol. 2, pp. 965-975, 2013. Available: http://dx.doi.org/10.1002/adhm.201200378

[111] P. Li, Z. Mao, Z. Peng, L. Zhou, Y. Chen, P.-H. Huang, et al., "Acoustic separation of circulating tumor cells," Proceedings of the National Academy of Sciences, vol. 112, pp. 4970-4975, 2015.

[112] D. R. Gossett, T. Henry, S. A. Lee, Y. Ying, A. G. Lindgren, O. O. Yang, et al., "Hydrodynamic stretching of single cells for large population mechanical phenotyping," Proceedings of the National Academy of Sciences, vol. 109, pp. 7630-7635, 2012.

[113] J. M. Munson and A. C. Shieh, "Interstitial fluid flow in cancer: implications for disease progression and treatment," Cancer Management and Research, vol. 6, pp. 317-328, 08/19 2014. Available: http://www.ncbi.nlm.nih.gov/pmc/articles/PMC4144982/

[114] Z. N. Demou, "Gene expression profiles in 3D tumor analogs indicate compressive strain differentially enhances metastatic potential," Ann Biomed Eng, vol. 38, pp. 3509-20, Nov 2010.

[115] A. Ostman and M. Augsten, "Cancer-associated fibroblasts and tumor growth-bystanders turning into key players," Curr Opin Genet Dev, vol. 19, pp. 67-73, Feb 2009.

[116] E. H. Starling, "On the absorption of fluids from the connective tissue spaces," The Journal of physiology, vol. 19, pp. 312-326, 1896. 
[117] J. B. West, "Respiratory physiology: the essentials," 9th edition ed: Wolters Kluwer Health, 2012, p. 177.

[118] H. Darcy, "Les fontaines publiques de la ville de Dijon, 1856," Dalmont, Paris, vol. 70.

[119] J. D. Jansen, "Porous-Media Flow," in A Systems Description of Flow Through Porous Media, ed: Springer, 2013, pp. 1-37.

[120] M. C. Moghadam, A. Deyranlou, A. Sharifi, and H. Niazmand, "Numerical simulation of the tumor interstitial fluid transport: Consideration of drug delivery mechanism," Microvascular Research, vol. 101, pp. 62-71, 2015. Available: http://dx.doi.org/10.1016/j.mvr.2015.06.007

[121] M. Mohammadi and P. Chen, "Effect of microvascular distribution and its density on interstitial fluid pressure in solid tumors: a computational model,"

Microvascular research, vol. 101, pp. 26-32, 2015. Available: http://dx.doi.org/10.1016/j.mvr.2015.06.001

[122] L. G. Griffith and M. A. Swartz, "Capturing complex 3D tissue physiology in vitro," Nature reviews Molecular cell biology, vol. 7, pp. 211-224, 2006.

[123] F. Pampaloni, E. G. Reynaud, and E. H. Stelzer, "The third dimension bridges the gap between cell culture and live tissue," Nature reviews Molecular cell biology, vol. 8, pp. 839-845, 2007.

[124] A. Abbott, "Cell culture: biology's new dimension," Nature, vol. 424, pp. 870-872, 2003.

[125] R. Z. Lin and H. Y. Chang, "Recent advances in three-dimensional multicellular spheroid culture for biomedical research," Biotechnology Journal, vol. 3, pp. 1172-1184, 2008.

[126] E. Curcio, S. Salerno, G. Barbieri, L. De Bartolo, E. Drioli, and A. Bader, "Mass transfer and metabolic reactions in hepatocyte spheroids cultured in rotating wall gas-permeable membrane system," Biomaterials, vol. 28, pp. 5487-5497, 2007.

[127] R. Narayanan, A. Rink, C. W. Beattie, and W.-S. Hu, "Differential gene expression analysis during porcine hepatocyte spheroid formation," Mammalian genome, vol. 13, pp. 515-523, 2002.

[128] N. E. Timmins, T. L. Maguire, S. M. Grimmond, and L. K. Nielsen, "Identification of three gene candidates for multicellular resistance in colon carcinoma," Cytotechnology, vol. 46, pp. 9-18, 2004.

[129] E. Gottfried, L. A. Kunz-Schughart, R. Andreesen, and M. Kreutz, "Brave little world: spheroids as an in vitro model to study tumor-immune-cell interactions," Cell Cycle, vol. 5, pp. 691-695, 2006.

[130] P. Seidl, R. Huettinger, R. Knuechel, and L. A. Kunz-Schughart, "Three-dimensional fibroblast-tumor cell interaction causes downregulation of RACK1 mRNA expression in breast cancer cells in vitro," International journal of cancer, vol. 102, pp. 129-136, 2002.

[131] L. A. Kunz-Schughart, P. Heyder, J. Schroeder, and R. Knuechel, "A heterologous 3 -D coculture model of breast tumor cells and fibroblasts to study tumorassociated fibroblast differentiation," Experimental cell research, vol. 266, pp. 7486, 2001.

[132] O. Oudar, "Spheroids: relation between tumour and endothelial cells," Critical reviews in oncology/hematology, vol. 36, pp. 99-106, 2000.

[133] M. S. Steinberg, "Reconstruction of tissues by dissociated cells," Science, vol. 141, pp. 401-408, 1963. 
[134] K. Guevorkian, M.-J. Colbert, M. Durth, S. Dufour, and F. Brochard-Wyart, "Aspiration of biological viscoelastic drops," Physical review letters, vol. 104, p. 218101, 2010.

[135] K. Guevorkian, D. Gonzalez-Rodriguez, C. Carlier, S. Dufour, and F. BrochardWyart, "Mechanosensitive shivering of model tissues under controlled aspiration," Proceedings of the National Academy of Sciences, vol. 108, pp. 13387-13392, August 16, 2011 2011. Available: http://www.pnas.org/content/108/33/13387.abstract

[136] S. Douezan, K. Guevorkian, R. Naouar, S. Dufour, D. Cuvelier, and F. BrochardWyart, "Spreading dynamics and wetting transition of cellular aggregates,"

Proceedings of the National Academy of Sciences, vol. 108, pp. 7315-7320, 2011.

[137] S. Douezan, J. Dumond, and F. Brochard-Wyart, "Wetting transitions of cellular aggregates induced by substrate rigidity," Soft Matter, vol. 8, pp. 4578-4583, 2012.

[138] D. Gonzalez-Rodriguez, L. Bonnemay, J. Elgeti, S. Dufour, D. Cuvelier, and F. Brochard-Wyart, "Detachment and fracture of cellular aggregates," Soft Matter, vol. 9, pp. 2282-2290, 2013. Available: http://dx.doi.org/10.1039/C2SM26648B

[139] D. Gonzalez-Rodriguez, K. Guevorkian, S. Douezan, and F. Brochard-Wyart, "Soft matter models of developing tissues and tumors," Science, vol. 338, pp. 910917, 2012. Available: http://dx.doi.org/10.1126/science.1226418

[140] W. Birchmeier and J. Behrens, "Cadherin expression in carcinomas: role in the formation of cell junctions and the prevention of invasiveness," Biochimica et Biophysica Acta (BBA)-Reviews on Cancer, vol. 1198, pp. 11-26, 1994.

[141] T. A. Martin, L. Ye, A. J. Sanders, J. Lane, and W. G. Jiang, "Cancer invasion and metastasis: molecular and cellular perspective," 2000.

[142] W. G. Stetler-Stevenson, S. Aznavoorian, and L. A. Liotta, "Tumor cell interactions with the extracellular matrix during invasion and metastasis," Annual review of cell biology, vol. 9, pp. 541-573, 1993.

[143] D. M. Gilkes, G. L. Semenza, and D. Wirtz, "Hypoxia and the extracellular matrix: drivers of tumour metastasis," Nature Reviews Cancer, vol. 14, pp. 430439, 2014.

[144] Marcos, H. C. Fu, T. R. Powers, and R. Stocker, "Separation of microscale chiral objects by shear flow," Physical review letters, vol. 102, p. 158103, 2009.

[145] J. Seymour, T. Ahmed, Marcos, and R. Stocker, "A microfluidic chemotaxis assay to study microbial behavior in diffusing nutrient patches," Limnol. Oceanogr.: Methods, vol. 6, pp. 477-488, 2008.

[146] Marcos, C. Yang, K. Ooi, T. Wong, and J. Masliyah, "Frequency-dependent laminar electroosmotic flow in a closed-end rectangular microchannel," Journal of colloid and interface science, vol. 275, pp. 679-698, 2004.

[147] Marcos, C. Yang, T. N. Wong, and K. T. Ooi, "Dynamic aspects of electroosmotic flow in rectangular microchannels," International Journal of Engineering Science, vol. 42, pp. 1459-1481, 2004.

[148] A. Guan, A. Shenoy, R. Smith, and Z. Li, "Streamline based design guideline for deterministic microfluidic hydrodynamic single cell traps," Biomicrofluidics, vol. 9, p. 024103, 2015.

[149] G. B. Jeffery, "The motion of ellipsoidal particles immersed in a viscous fluid," in Proceedings of the Royal Society of London A: Mathematical, Physical and Engineering Sciences, 1922, pp. 161-179. 
[150] G. M. Whitesides, E. Ostuni, S. Takayama, X. Jiang, and D. E. Ingber, "Soft lithography in biology and biochemistry," Annual review of biomedical engineering, vol. 3, pp. 335-373, 2001.

[151] S. Marcos, "R.(2006) Microorganisms in vortices: a microfluidic setup," Limnology and Oceanography: Methods, vol. 4, pp. 392-398.

[152] R. Pantoja, J. M. Nagarah, D. M. Starace, N. A. Melosh, R. Blunck, F. Bezanilla, et al., "Silicon chip-based patch-clamp electrodes integrated with PDMS microfluidics," Biosensors and Bioelectronics, vol. 20, pp. 509-517, 2004.

[153] M. A. Unger, H.-P. Chou, T. Thorsen, A. Scherer, and S. R. Quake, "Monolithic microfabricated valves and pumps by multilayer soft lithography," Science, vol. 288, pp. 113-116, 2000.

[154] T. Deng, H. Wu, S. T. Brittain, and G. M. Whitesides, "Prototyping of masks, masters, and stamps/molds for soft lithography using an office printer and photographic reduction," Analytical chemistry, vol. 72, pp. 3176-3180, 2000.

[155] J. Ferlay, I. Soerjomataram, M. Ervik, R. Dikshit, S. Eser, C. Mathers, et al., "Cancer Incidence and Mortality Worldwide: IARC CancerBase No. 11 [Internet]. GLOBOCAN 2012 v10. Lyon, France: International Agency for Research on Cancer, 2013," ed, 2012.

[156] R. M. Neve, K. Chin, J. Fridlyand, J. Yeh, F. L. Baehner, T. Fevr, et al., "A collection of breast cancer cell lines for the study of functionally distinct cancer subtypes," Cancer cell, vol. 10, pp. 515-527, 2006.

[157] D. L. Holliday and V. Speirs, "Choosing the right cell line for breast cancer research," Breast Cancer Research, vol. 13, p. 1, 2011.

[158] H. Schatten, Cell and molecular biology of breast cancer: Springer, 2013.

[159] P. L. Ryan, R. A. Foty, J. Kohn, and M. S. Steinberg, "Tissue spreading on implantable substrates is a competitive outcome of cell-cell vs. cell-substratum adhesivity," Proceedings of the National Academy of Sciences, vol. 98, pp. 43234327, 2001.

[160] S. Zheng, H. Lin, J.-Q. Liu, M. Balic, R. Datar, R. J. Cote, et al., "Membrane microfilter device for selective capture, electrolysis and genomic analysis of human circulating tumor cells," Journal of chromatography A, vol. 1162, pp. 154$161,2007$.

[161] S. C. Dolfi, L. L.-Y. Chan, J. Qiu, P. M. Tedeschi, J. R. Bertino, K. M. Hirshfield, et al., "The metabolic demands of cancer cells are coupled to their size and protein synthesis rates," Cancer \& metabolism, vol. 1, p. 1, 2013.

[162] E. L. Persons, "STUDIES ON RED BLOOD CELL DIAMETER: III. The Relative Diameter of Immature (Reticulocytes) and Adult Red Blood Cells in Health and Anemia, Especially in Pernicious Anemia," Journal of Clinical Investigation, vol. 7, p. 615, 1929.

[163] J.-M. Paulus, "Platelet size in man," Blood, vol. 46, pp. 321-336, 1975.

[164] E. P. Solomon, Introduction to human anatomy and physiology: Elsevier Health Sciences, 2015.

[165] R. L. Eifler, J. Lind, D. Falkenhagen, V. Weber, M. B. Fischer, and R. Zeillinger, "Enrichment of circulating tumor cells from a large blood volume using leukapheresis and elutriation: proof of concept," Cytometry Part B: Clinical Cytometry, vol. 80, pp. 100-111, 2011.

[166] R. Gertler, R. Rosenberg, K. Fuehrer, M. Dahm, H. Nekarda, and J. Siewert, "Molecular Staging of Cancer," ed: Springer, 2003.

[167] S. Suresh, "Biomechanics and biophysics of cancer cells," Acta Materialia, vol. 55, pp. 3989-4014, 2007. 
[168] V. Swaminathan, K. Mythreye, E. T. O'Brien, A. Berchuck, G. C. Blobe, and R. Superfine, "Mechanical stiffness grades metastatic potential in patient tumor cells and in cancer cell lines," Cancer research, vol. 71, pp. 5075-5080, 2011.

[169] J. M. Barnes, J. T. Nauseef, and M. D. Henry, "Resistance to fluid shear stress is a conserved biophysical property of malignant cells," PloS one, vol. 7, p. e50973, 2012.

[170] R. H. Lam, Y. Sun, W. Chen, and J. Fu, "Elastomeric microposts integrated into microfluidics for flow-mediated endothelial mechanotransduction analysis," Lab on a Chip, vol. 12, pp. 1865-1873, 2012.

[171] Z. Wang, M.-C. Kim, M. Marquez, and T. Thorsen, "High-density microfluidic arrays for cell cytotoxicity analysis," Lab on a Chip, vol. 7, pp. 740-745, 2007.

[172] M. E. Piyasena, P. P. Austin Suthanthiraraj, R. W. Applegate Jr, A. M. Goumas, T. A. Woods, G. P. López, et al., "Multinode acoustic focusing for parallel flow cytometry," Analytical chemistry, vol. 84, pp. 1831-1839, 2012.

[173] S. Hu, G. Liu, W. Chen, X. Li, W. Lu, R. H. Lam, et al., "Multiparametric Biomechanical and Biochemical Phenotypic Profiling of Single Cancer Cells Using an Elasticity Microcytometer," Small, 2016.

[174] P. Grover, A. Cummins, T. Price, I. Roberts-Thomson, and J. Hardingham, "Circulating tumour cells: the evolving concept and the inadequacy of their enrichment by EpCAM-based methodology for basic and clinical cancer research," Annals of Oncology, vol. 25, pp. 1506-1516, 2014.

[175] P. C. Carman, Flow of gases through porous media: Academic press, 1956.

[176] R. P. Chapuis and M. Aubertin, Predicting the coefficient of permeability of soils using the Kozeny-Carman equation: École polytechnique de Montréal, 2003.

[177] J. Kozeny, Über kapillare Leitung des Wassers im Boden:(Aufstieg, Versickerung und Anwendung auf die Bewässerung): Hölder-Pichler-Tempsky, 1927.

[178] C. P. Ng, B. Hinz, and M. A. Swartz, "Interstitial fluid flow induces myofibroblast differentiation and collagen alignment in vitro," Journal of cell science, vol. 118, pp. 4731-4739, 2005.

[179] H. Phillips and M. Steinberg, "Embryonic tissues as elasticoviscous liquids. I. Rapid and slow shape changes in centrifuged cell aggregates," Journal of cell science, vol. 30, pp. 1-20, 1978.

[180] M. W. Toepke and D. J. Beebe, "PDMS absorption of small molecules and consequences in microfluidic applications," Lab on a Chip, vol. 6, pp. 1484-1486, 2006.

[181] R. Mukhopadhyay, "When PDMS isn't the best," Analytical chemistry, vol. 79, pp. 3248-3253, 2007.

[182] J. Tien, J. G. Truslow, and C. M. Nelson, "Modulation of invasive phenotype by interstitial pressure-driven convection in aggregates of human breast cancer cells," PloS one, vol. 7, p. e45191, 2012.

[183] W. K. Peng, T. F. Kong, C. S. Ng, L. Chen, Y. Huang, A. A. S. Bhagat, et al., "Micromagnetic resonance relaxometry for rapid label-free malaria diagnosis," Nature medicine, vol. 20, pp. 1069-1073, 2014. 


\section{Appendix}

\section{Appendix 1: Gelling procedure for type I collagen gel}

1) The collagen gel used in this procedure is Collagen I, Bovine at initial concentration $5 \mathrm{mg} \mathrm{ml}^{-1}$ (A10644-01, Thermo Fisher Scientific, Singapore).

2) Place the collagen gel $\left(5 \mathrm{mg} \mathrm{ml}^{-1}\right)$, sterile 10X PBS solution, sterile distilled water and sterile $1 \mathrm{~N} \mathrm{NaOH}$ on ice.

3) Determine the concentration and final volume of collagen needed $\left(V_{l}\right)$ :

$$
V_{1}=\frac{(\text { Final concentration of collagen }) \times(\text { Total volume })}{\text { Initial concentration of collagen }}
$$

4) Determine the amount of $10 \mathrm{X}$ PBS solution $\left(V_{2}\right), 1 \mathrm{~N} \mathrm{NaOH}\left(V_{3}\right)$ and distilled water $\left(V_{4}\right)$ needed:

$$
\begin{gathered}
V_{2}=\frac{\text { Total Volume }}{10} . \\
V_{3}=V_{1} \times 0.025 . \\
V_{4}=(\text { Total Volume })-\left(V_{1}+V_{2}+V_{3}\right) .
\end{gathered}
$$

5) Mix the needed amount of distilled water, $1 \mathrm{~N} \mathrm{NaOH}$ and 10X PBS in a sterile centrifuge tube.

6) Slowly inject the needed amount of collagen into the centrifuge tube and gently mix the solution by pipetting up and down several times. 
7) Using pipette to inject the collagen solution to the desired microfluidic device. Note: gelling occurs rapidly at room temperature.

8) Place the device filled with the collagen solution inside an incubator at $37^{\circ} \mathrm{C}$ for at least 30 minutes for the gel to be formed.

\section{Appendix 2: Device preparation for the calibration test of the second design to characterize flow through a cellular aggregate}

In this setup, we need to block the main channel of the second design (discussed in 3.4) so that when applying flow to the device, all the flow will only go through the side channel. This helps us quantify the hydrodynamic resistance of the side channel. As we want all the side channels in our experiment to be identical, we replicate our devices by molding the same pattern in the wafer master mold and use one device for the calibration test.

1) The second design is fabricated by photo-lithography and molded in PDMS.

2) The PDMS chip will be punched holes and placed on a wafer so that the microchannel side of the PDMS chip contacts with the wafer surface. Gently press the PDMS chip so that all the surface of the PDMS is in contact with the wafer.

Note: No plasma bonding is done in this step.

3) A droplet of $\sim 30 \mu 1$ PDMS solution (the ratio between PDMS monomer and curing agent is 10:1) is prepared and injected to the channel 2 inlet of the second design PDMS chip. Under an upright microscope, we observe the PDMS solution slowly move through the microchannel 2 to the chamber. 
4) When the PDMS solution fill up all the chamber space, place the wafer with the PDMS chip on top inside an oven at $80{ }^{\circ} \mathrm{C}$ for one hour. The PDMS solution inside the chamber gets cured and results in a PDMS blockage at the chamber.

5) The PDMS chip is then peeled off the wafer and bonded with a glass slide using plasma cleaner. This helps secure the adhesion of the PDMS blockage on the glass slide. When apply flow to the main channel, the flow only goes through the side channel.
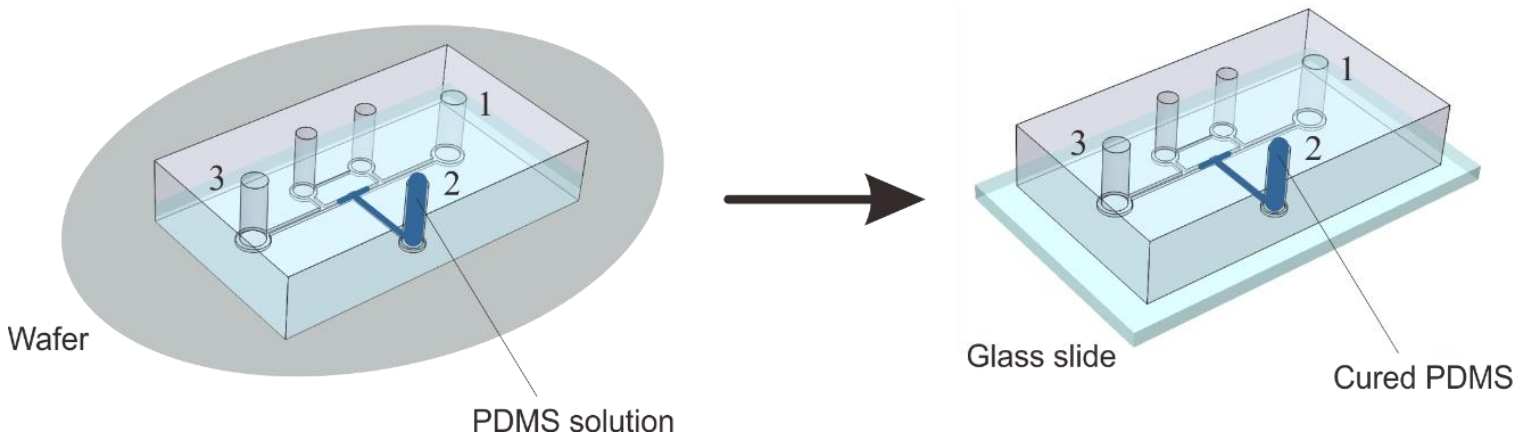

Figure A.1 Schematics of the preparation for the calibration test of the second design to characterize interstitial flow through a cellular aggregate. 


\section{Publication}

\section{Journal publication}

1. Q. D. Tran, T. F. Kong, D. Hu, Marcos, and R. H. Lam, "Deterministic sequential isolation of floating cancer cells under continuous flow," Lab on a Chip, 2016.

\section{Conference}

1. Tran Duc Quang, Marcos, David Gonzalez-Rodriguez, "Microfluidic system for studying interstitial flow through a model tumor,"

BIOMS | EMBL Conference: Physics of Cells and Tissues 2015 - Modelling meets Experiment, EMBL Heidelberg, Germany, 2015.

2. Tran Duc Quang, Kong Tian Fook, Marcos, and Lam H.W. Raymond, "Microfluidics for sequential particle trapping," ASME 2016 Micro/Nanoscale Heat \& Mass Transfer International Conference, Biopolis, Singapore, 2016. 